\title{
Image Processing Algorithms for Patient Mobility Monitoring using Pressure Sensitive Bed and Floor Arrays
}

\author{
By
}

Matthew Taylor, B. Eng.

A thesis submitted to the Faculty of Graduate Studies and Research in partial fulfillment of the requirements for the degree of

Master of Applied Science in Biomedical Engineering

Ottawa-Carleton Institute for Biomedical Engineering (OCIBME)

Department of Systems and Computer Engineering

Carleton University

Ottawa, Ontario, Canada, K1S 5B6

October 2012

(c) Copyright 2012, Matthew Taylor 
Library and Archives

Canada

Published Heritage

Branch

395 Wellington Street

Ottawa ON K1A ON4

Canada
Bibliothèque et

Archives Canada

Direction du

Patrimoine de l'édition

395 , rue Wellington

Ottawa ON K1A ON4

Canada
Your file Votre référence

ISBN: 978-0-494-93504-0

Our file Notre référence

ISBN: $978-0-494-93504-0$
NOTICE:

The author has granted a nonexclusive license allowing Library and Archives Canada to reproduce, publish, archive, preserve, conserve, communicate to the public by telecommunication or on the Internet, loan, distrbute and sell theses worldwide, for commercial or noncommercial purposes, in microform, paper, electronic and/or any other formats.

The author retains copyright ownership and moral rights in this thesis. Neither the thesis nor substantial extracts from it may be printed or otherwise reproduced without the author's permission.
AVIS:

L'auteur a accordé une licence non exclusive permettant à la Bibliothèque et Archives Canada de reproduire, publier, archiver, sauvegarder, conserver, transmettre au public par télécommunication ou par l'Internet, prêter, distribuer et vendre des thèses partout dans le monde, à des fins commerciales ou autres, sur support microforme, papier, électronique et/ou autres formats.

L'auteur conserve la propriété du droit d'auteur et des droits moraux qui protege cette thèse. $\mathrm{Ni}$ la thèse ni des extraits substantiels de celle-ci ne doivent être imprimés ou autrement reproduits sans son autorisation.
In compliance with the Canadian Privacy Act some supporting forms may have been removed from this thesis.

While these forms may be included in the document page count, their removal does not represent any loss of content from the thesis.
Conformément à la loi canadienne sur la protection de la vie privée, quelques formulaires secondaires ont été enlevés de cette thèse.

Bien que ces formulaires aient inclus dans la pagination, il n'y aura aucun contenu manquant. 


\section{Abstract}

This thesis focuses on the problem of unobtrusive patient monitoring using pressure sensor arrays to help assess the mobility of older adults. Using under mattress pressure sensor arrays data is captured continuously from occupants. The raw data is processed using specialized algorithms and important clinical information is extracted. Key clinical features including bed entry time, sleep duration, number of exits, and bed exit time (lie-to-sit and sit-to-stand times) can be extracted. These features can be used by clinicians to assist in assessing the patient's mobility. A floor tile based pressure sensor array is used to capture data while the patient stands still on it and shifts their weight. The data is processed and clinical information is extracted related to the patient's static and dynamic balance. Key clinical features include weight balancing, center of pressure under the feet, and amount of sway, assist in assessing the patient's standing stability and static balance. Average and maximum movement velocity measures can assist in assessing the patient's dynamic balance. The thesis shows that pressure sensor arrays can be used to collect data unobtrusively and using specialized image processing algorithms, clinically relevant information can be extracted which can assist in assessing the mobility and stability of older adults. 


\section{Acknowledgements}

I would like to thank everyone that has stood by me in this journey of higher education. Dr. Rafik Goubran was been a superb thesis supervisor, who has given me all the opportunities to expand my knowledge in the research world. Dr. Frank Knoefel has been an excellent medical co-supervisor, providing much needed clinical insight to the project. The research team at Bruyère Continuing Care have been at the forefront of collecting real patient data and helped with the clinical interpretations of the results. My colleagues in the DSP lab have kept the work environment stimulating and lively on a regular basis; thanks Vilas, Luke, Melanie, and Rania. I would like to thanks my parents for giving me the opportunity to become a graduate student and live at home for so long! Finally I would like to thank Ruqia, she has been by my side throughout my undergrad and grad career, helping me through those tough times and sleepless nights. 


\section{Contents}

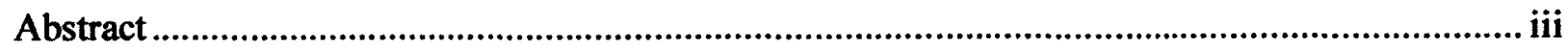

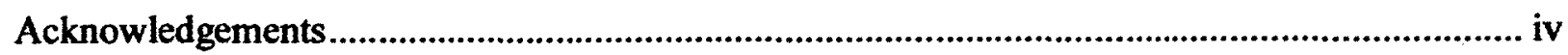

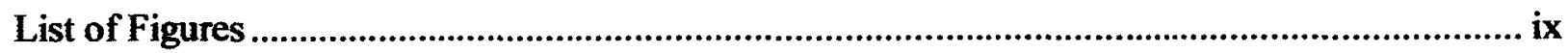

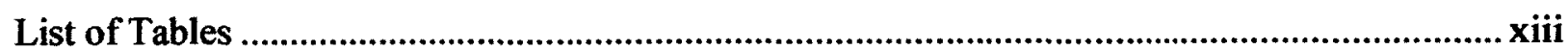

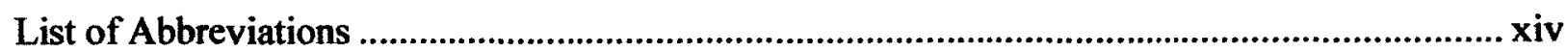

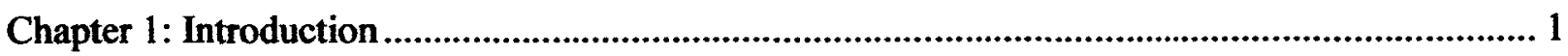

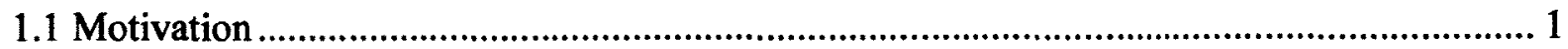

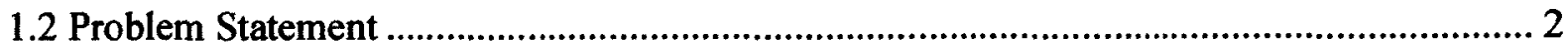

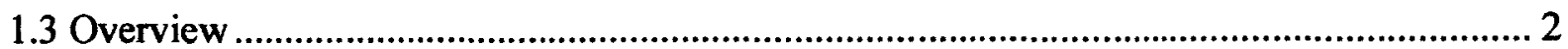

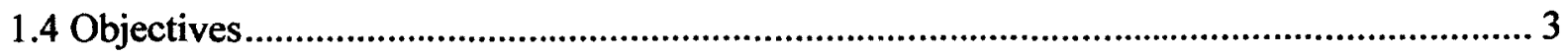

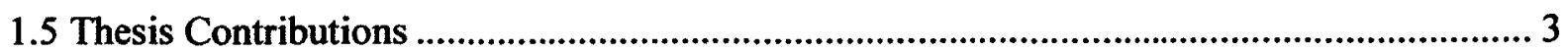

1.6 Thesis Organization.................................................................................................... 5

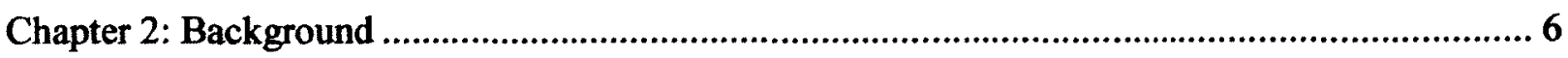

2.1 Introduction

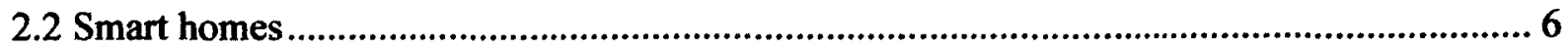

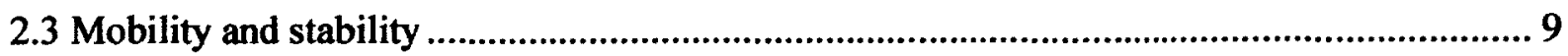




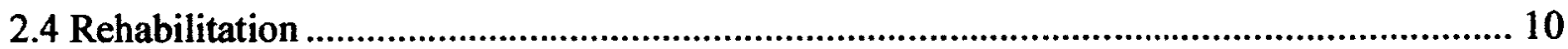

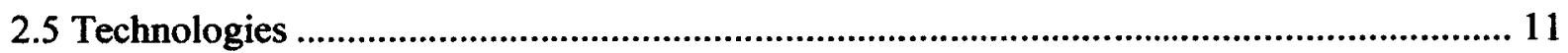

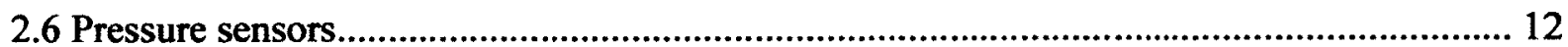

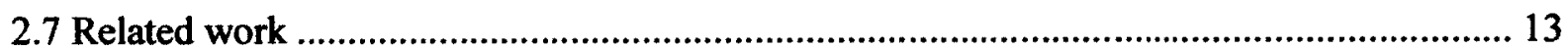

Chapter 3: Experiment Setup ............................................................................................... 16

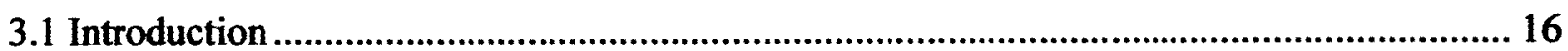

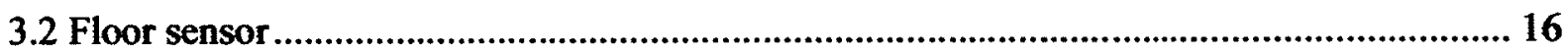

3.2.1 Hardware …....................................................................................................... 17

3.2.2 Equipment setup …............................................................................................ 18

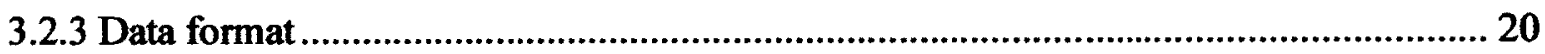

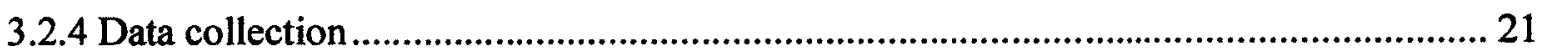

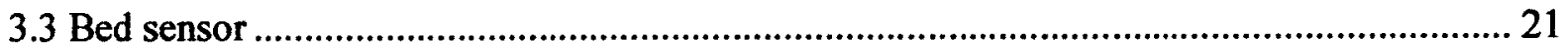

3.3.1 Hardware …

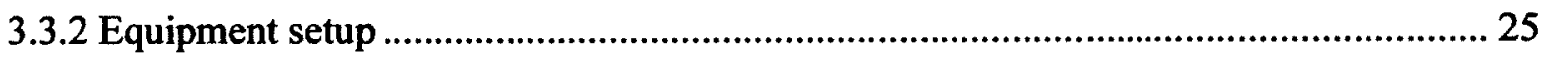

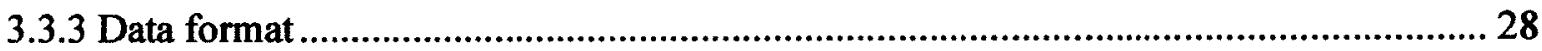

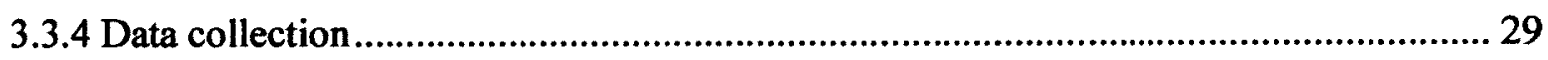

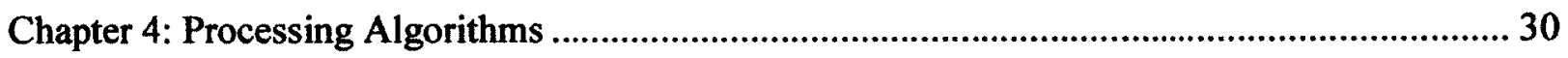

4.1 Floor sensor

4.1.1 Generating pressure map images .............................................................................. 30

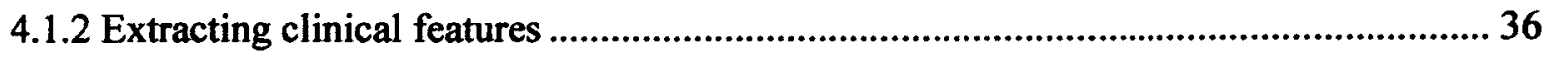




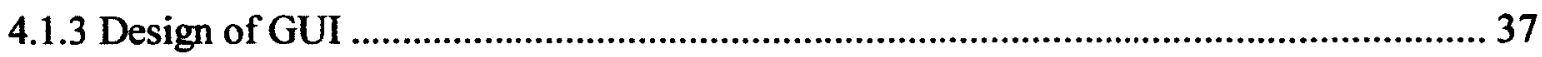

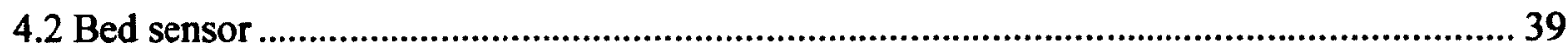

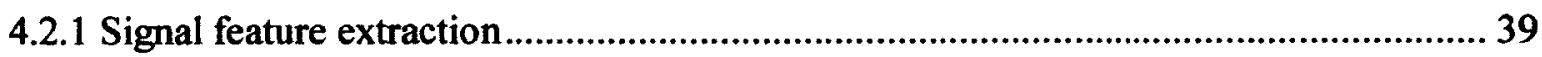

4.2.2 Clinical feature extraction ..................................................................................... 51

4.2.3 Exporting data, plots, images .................................................................................6

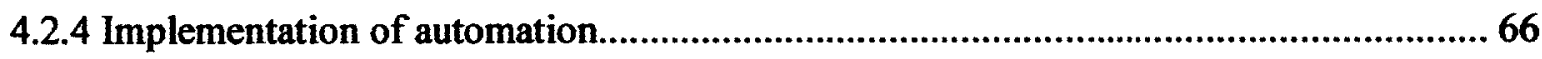

4.2.5 Feature comparison and performance..................................................................... 80

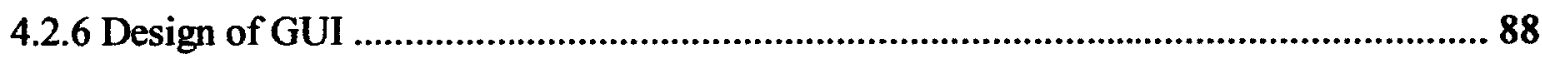

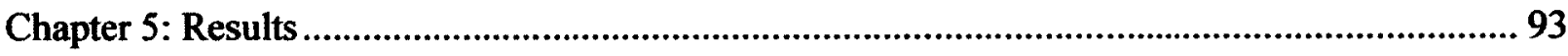

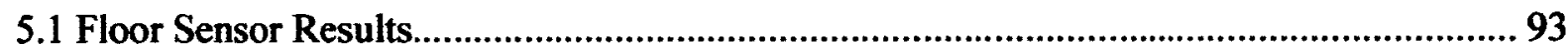

5.1.1 Older adult compared to younger adult ..................................................................... 96

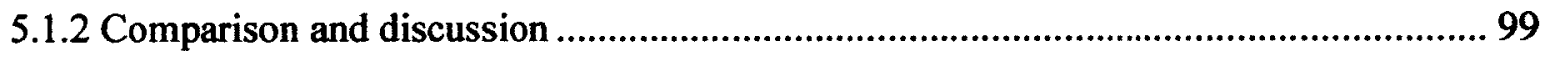

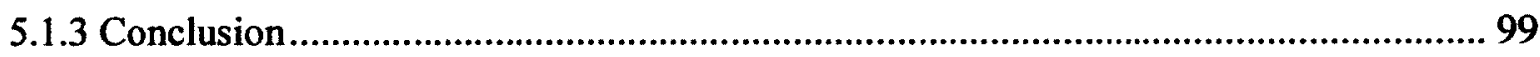

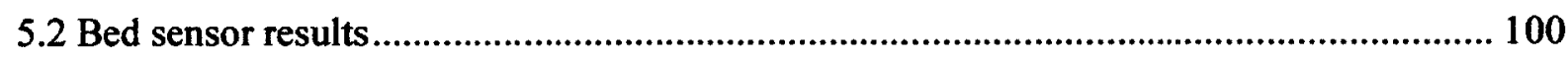

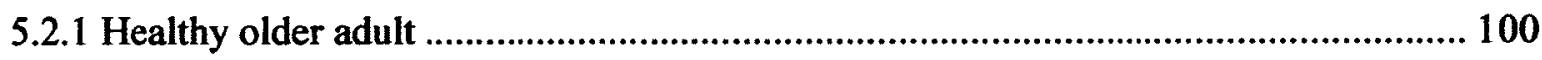

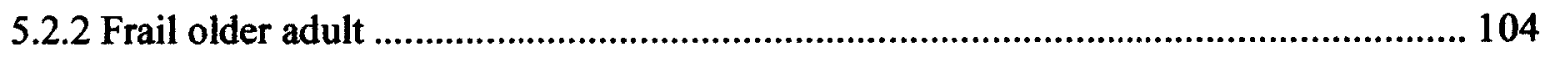

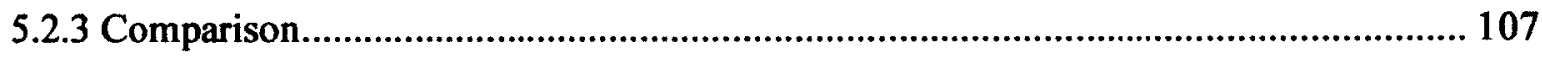

5.3 Combination of sensors ............................................................................................ 108

Chapter 6: Conclusion.......................................................................................................... 109

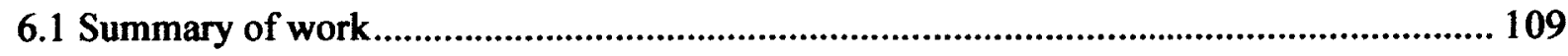


6.3 Future work

References 


\section{List of Figures}

Figure 1: Inside the TAFETA apartment [14] ................................................................... 8

Figure 2: Inside the TAFETA apartment; other sensors in use [14]........................................... 8

Figure 3: Floor tile pressure sensor with person standing on it ................................................... 18

Figure 4: Equipment setup for floor tile pressure sensor ............................................................... 19

Figure 5: Tactex data capture interface for floor tile pressure sensor............................................ 19

Figure 6: Bed mat pressure sensor array ..................................................................................... 23

Figure 7: Illustrated layout of individual sensors (red) in the bed pressure mat. Circuit board is

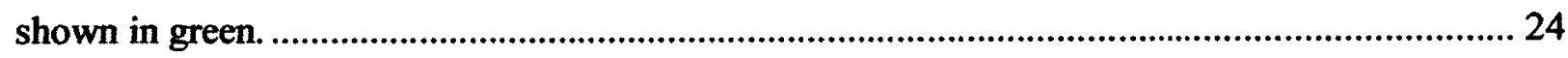

Figure 8: Bluetooth transmitter unit for bed mat sensor ............................................................... 24

Figure 9: Pressure mat orientation under mattress. Pressure sensor is in blue and shows the three

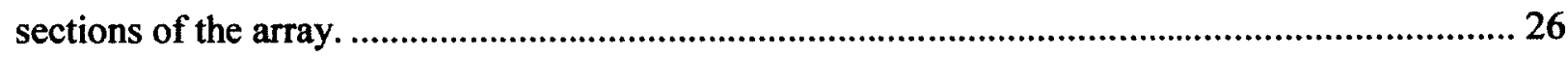

Figure 10: Side view of equipment install under mattress........................................................... 26

Figure 11: Pressure mapped image created from $50 \mathrm{~Hz}$ sampling of patient standing on pressure

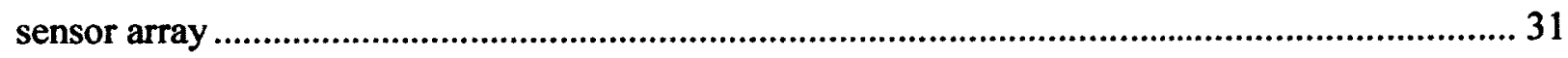

Figure 12: Pressure mapped image created from $300 \mathrm{~ms}$ averaging of patient standing on pressure

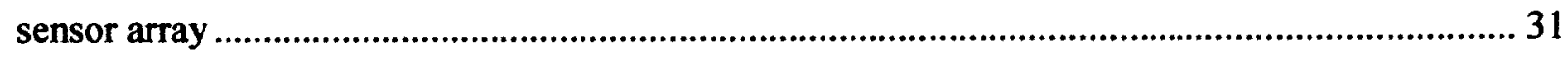

Figure 13: Flow chart for proposed algorithm to measure stability.............................................. 33

Figure 14: Processed images of patient on sensor at varying image resolutions with centers of

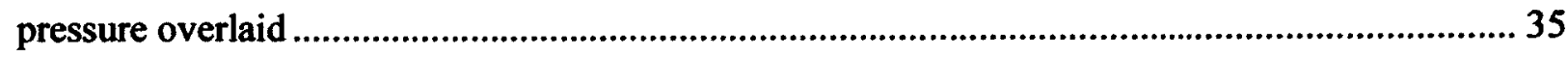




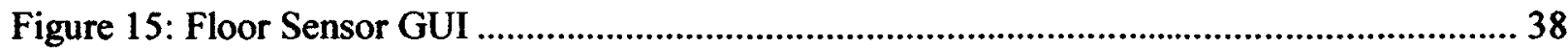

Figure 16: Pressure mapped image showing concentrated pressure (person sitting) .................. 41

Figure 17: Pressure mapped image showing dispersion (person lying) .............................. 41

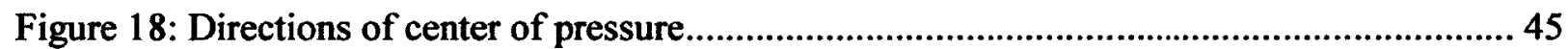

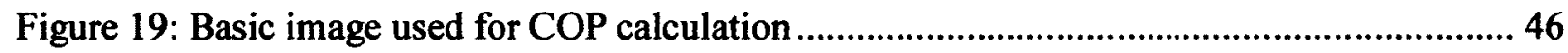

Figure 20: Basic image for COP calculation solved. COP in red.......................................... 49

Figure 21: Example of features for 24 hour recording from P382 .................................... 50

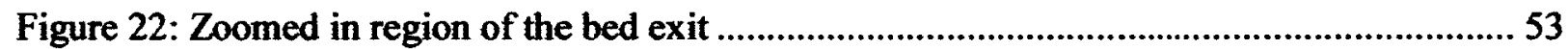

Figure 23: Zoomed in region of the bed exitwith annotations.............................................. 53

Figure 24: Bed exit transfer using COP-x feature .......................................................... 55

Figure 25: Bed exit transfer using COP-x feature. Annotated with points of interest................. 56

Figure 26: Pressure mapped images of patient lying in state $\mathrm{X} 1$..................................... 58

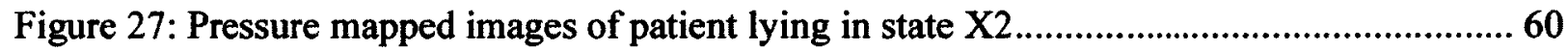

Figure 28: Pressure mapped images of patient lying in state X2.5

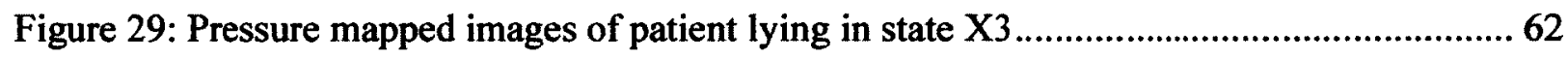

Figure 30: Pressure mapped images of patient in sitting position in state $\mathrm{X} 4$........................... 63

Figure 31: Pressure mapped images of patient out of bed, state X5 ...................................... 64

Figure 32: Flowchart of automated processing algorithm for bed mat sensor .........................67

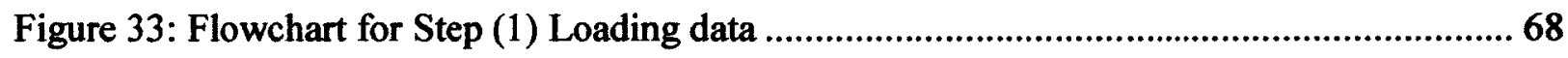

Figure 34: Flowchart for Step (2) Preprocessing data ....................................................... 71

Figure 35: Flowchart of Step (3) Extracting features ....................................................... 72

Figure 36: Probability distribution of features showing separability of states ........................ 73

Figure 37: Stages of the bed exit. Manually selected .......................................................... 74 
Figure 38: Flowchart of Step (4) Extracting clinical information from features. 75

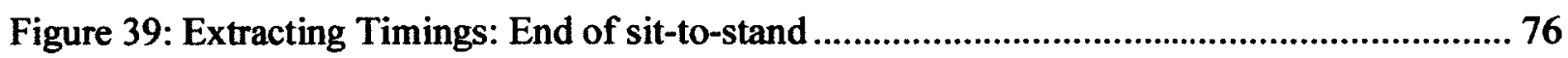

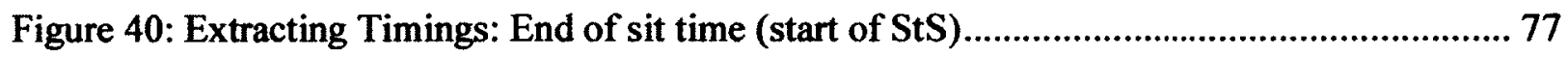

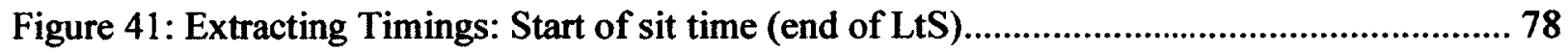

Figure 42: Extracting Timings: End of lie time (start of LtS) .......................................... 80

Figure 43: Bed exit 1 using automatic timing extraction. data_sn4352_2011_12_09_01_18_5781

Figure 44: Bed exit 2 using automatic timing extraction. data_sn4352_2011_12_10_10_19_3082

Figure 45: Bed exit 3 using automatic timing extraction. data_sn4352_2011_12_10_13_19_3383

Figure 46: Bed exit 4 using automatic timing extraction. data_sn4352_2011_12_11_09_19_5384

Figure 47: Bed exit 5 using automatic timing extraction. data_sn4352_2011_12_12_07_20_1585

Figure 48: Bed exit 6 using automatic timing extraction. data_sn4352_2011_12_13_06_20_38 86

Figure 49: Bed exit 7 using automatic timing extraction. data_sn4352_2011_12_13_11_20_4387

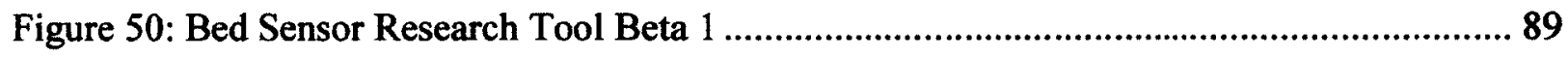

Figure 51: Bed Sensor Main Control Center .................................................................. 91

Figure 52: Young adult standing on sensor array with balanced pressure under each foot and

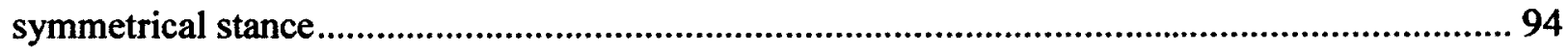

Figure 53: Example of COP over time for an older adult with standing stability issues.............95

Figure 54: Example of COP over time for a young adult without standing stability issues........ 96

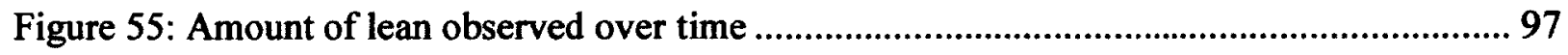

Figure 56: Older adult standing on sensor array. Calculated center of pressures shown illustrating an asymmetrical stance with greater weight on the left foot. .............................................98

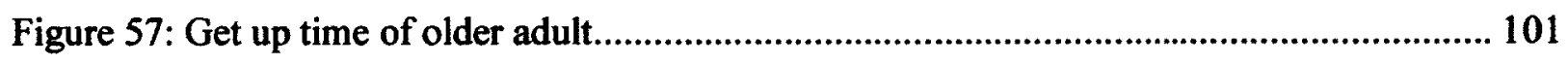

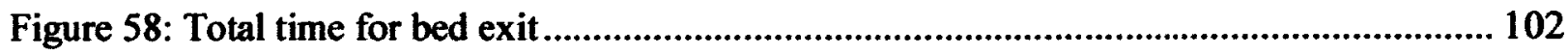


Figure 59: Time to sit up from a lying position ................................................................ 102

Figure 60: Time spent in the sitting position ........................................................................ 103

Figure 61: Time to stand up from sitting position ................................................................... 103

Figure 62: Functional Performance Measures ........................................................................... 105

Figure 63: Time to sit up from a lying position ........................................................................... 106

Figure 64: Time spent in the sitting position ......................................................................... 106

Figure 65: Time to stand up from sitting position ................................................................... 107 


\section{List of Tables}

Table 1: Floor sensor data example

Table 2: Bed sensor data format example ................................................................................. 28

Table 3: Matrix representation of basic image for COP calculation ................................................ 46

Table 4: Matrix for solving COP in $x$ direction ......................................................................... 47

Table 5: Matrix for solving COP in y direction .......................................................................... 47

Table 6: Modified solving matrix for COP $x$ direction.................................................................... 47

Table 7: Modified solving matrix for COP y direction................................................................48

Table 8: Example showing bed occupancy features................................................................ 52

Table 9: Bed occupancy detection results..................................................................................69

Table 10: Bed exit 1 extracted timings .................................................................................. 81

Table 11: Bed exit 2 extracted timings ....................................................................................... 82

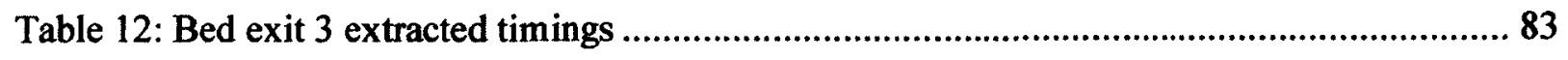

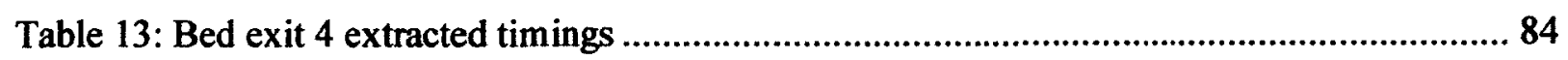

Table 14: Bed exit 5 extracted timings …………………………........................................... 85

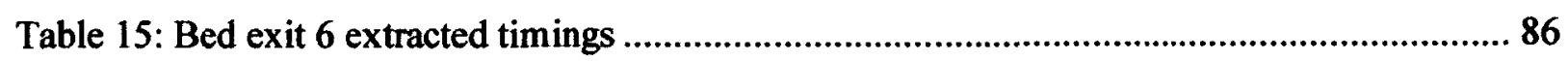

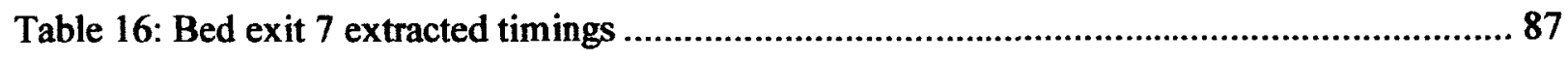

Table 17: Bed exit timings for older adult ............................................................................. 101 


\section{List of Abbreviations}

\begin{tabular}{|l|l|}
\hline Abbreviation & Definition \\
\hline COP & Center of Pressure \\
\hline COP-x & X coordinate (Horizontal) of COP \\
\hline COP-y & Y coordinate (Vertical) of COP \\
\hline StS & Sit-to-Stand \\
\hline LtS & Lie-to-Sit \\
\hline PrS & Pressure Sum \\
\hline TAFETA & Technology Assisted Friendly Environment for the Third Age \\
\hline
\end{tabular}




\section{Chapter 1: Introduction}

\subsection{Motivation}

The elderly population is growing and this creates an increased strain on health care resources. Canada's population of seniors (65 years and over) was at $14.4 \%$ in 2011 , this is expected to increase to $18.5 \%$ by 2021 , and over $25 \%$ by 2061 [1]. If elderly people are able to age at home with fewer hospital visits this will allow the limited healthcare resources to benefit more users. The people themselves will benefit staying in a place they are familiar with and secure in [2].

Technology is a rapidly growing industry and more and more sensors and devices are entering the market daily. Modern technology can be used to assist elderly patients to live a meaningful life in the comfort of their own home. Pressure sensitive technology is unobtrusive and can be used to monitor many aspects of a person's daily life [3]. For instance pressure sensors incorporated in the bed can measure how long they spend in bed; sensors on the floor can perform gait analysis to get an idea of the person's mobility.

Using these sensors can benefit elderly adults who wish to age at home. 


\subsection{Problem Statement}

Pressure sensors have been used in the past for patient monitoring and extracting meaningful clinical information such as respiratory rate and sit-to-stand times [4], [5]. The problem is how to extract relevant clinical information related to patient mobility monitoring using image processing algorithms. Data is recorded in a real world environment using two different pressure sensor devices. Custom algorithms must be developed for each to extract data in an autonomous way. This thesis will focus on the signal processing techniques used and the resulting clinical information.

\subsection{Overview}

The sensors used include a floor title which contains 2304 sensors which patients stand on and a bed mat which contains 72 sensors which the patients sleep on. Specialized signal processing algorithms were developed for both sensor arrays to extract meaningful clinical information. The processed data from the floor sensor includes clinical measures to assist in assessing the patient's standing stability, such as the patient's center of pressure, load distribution, static and dynamic balance. The processed data from the bed mat sensor includes clinical measures to assist in assessing the patient's mobility, such as the amount of time required to enter and exit the bed, the symmetry of the exit, and any failed attempts. It can also extract other information relevant to the 
patient's health, such as time spent in bed, number of exits during the night, and an estimate of tossing and turning during the night.

\subsection{Objectives}

To complete the thesis objective of autonomous patient monitoring by extracting key clinical information, the following methodological objective were identified:

- Record pressure signal data using both technologies from various participants.

- Examine pressure signals and find relevant signal features.

- Determine what relevant clinical information can be extracted from signal features; i.e. lie-to-sit and sit-to-stand times, and standing symmetry and stability.

- Determine best set of features for determining clinical information; i.e. timings based on summed pressure signal or center of pressure location.

- Design and implement an automated pressure signal processing algorithm.

- Evaluate performance of system and compare to results reported in related literature.

\subsection{Thesis Contributions}

The following contributions were made during the thesis research.

- A novel processing algorithm was developed to convert raw pressure data recorded by the floor pressure sensor into pressure mapped images for clinical analysis. Various 
sampling rates and resolutions were tested to determine minimum requirements to obtain high accuracy in the least amount of processing time [6]. The work was published in IEEE International Instrumentation and Measurement Technology Conference 2012 (I2MTC 2012) in Graz, Austria, May 2012.

- A standing stability system was designed using the original processing algorithm with the addition of a secondary one to extract clinical features that can assist in assessing a patient's standing stability. The system analyzes features related to static balance; the amount of lean, the center of pressure, the amount of sway while standing still and dynamic balance; the velocity at which they can shift their weight. The standing stability of an older adult with chronic stroke was assessed and compared to a healthy young adult [7]. The system and results were published in IEEE International Symposium on Medical Measurements and Applications Proceedings (MeMeA 2012) in Budapest, Hungary, May 2012.

- A MATLAB graphical user interface (GUI) was developed to assist researchers in analyzing the floor pressure sensor recordings. The GUI allows for quick processing with various options for data output easily configured.

- A new pressure sensitive mat was tested in a pilot project to assess its usability for long term bed pressure recordings. Data was processed and the quality was assessed. The mat was considered more than adequate for our research purposes. The work will be presented at the Regional Geriatric Conference of Eastern Ontario in Ottawa, Ontario, October 2012 [8]. 
- A novel processing algorithm was developed to extract meaningful clinical information from the pressure mat recordings. The processing algorithm is able to scan through $100 \mathrm{~s}$ of hours of recordings and extract meaningful information automatically and display that in a user friend way. One of the clinical features is the bed exit timing, data collected from an older adult was analyzed and the results will be presented at the Canadian Association on Gerontology Conference in Vancouver, British Columbia, October, 2012 [9].

- A MATLAB graphical user interface (GUI) was developed to assist researchers in analyzing the bed mat pressure sensor recordings. The GUI allows for quick processing with all options for data output easily changed.

\subsection{Thesis Organization}

This thesis is organized into six chapters. The first chapter is the introduction which has discussed the motivation, objectives, and contributions from the thesis. The second chapter will provide relevant background information before discussing the main content of the thesis. Chapter 3 discusses the experiment setup, including the hardware used and the data collection procedures. Chapter 4 discusses the image processing algorithms designed to extract the clinical information from the recorded data. Chapter 5 presents results of the processing algorithms use in extracting clinical information from healthy or young adults and older or frail adults. Chapter 6 concludes the thesis with a synopsis of the results and suggestions for future work. 


\section{Chapter 2: Background}

\subsection{Introduction}

This chapter reviews the background information and related work relevant to this thesis. This research relates to two themes, medical and engineering work. The medical aspect relates to smart homes, where the research equipment is installed, and the patients themselves. The mobility and stability of the patient is the focus of the clinical information, this information can be used to help in rehabilitation programs. The other theme is engineering; the main aspects of it are the technology, pressure sensor arrays, and the image processing techniques. Specialized processing algorithms are developed to extract clinical information.

\subsection{Smart homes}

Smart homes and the technology used within are a rapidly evolving area. It is an important field of research as people wish to have their living environment more intelligent and adaptive. 
Technology already exists to track habitants within their home allowing control of lighting and temperature conditions [10], [11]. Smart homes can be tailored with technology to provide patients with assisted living. Technology can provide health monitoring with the specific needs of the patient in mind [12]. These smart homes can allow for older adults to age comfortably at home with fewer visits to the hospital. This is the idea behind the Technology Assisted Friendly Environment for the Third Age (TAFETA) project which is a joint venture between Carleton University and Bruyère Continuing Care, a local health care provider specialized with seniors, rehabilitation and palliative care [13]. The project allows researchers to develop and test technology that can improve a patient's quality of life by responding to their health needs automatically. The smart home incorporates many types of sensors into the environment to monitor the patient's health and needs. Some of these sensors include microphone arrays which can detect cough sounds, electronic noses, and pressure sensitive arrays [14]. Figure 1 shows the bed where a pressure sensitive mat is installed, Figure 2 shows the couch where a pressure sensor was installed as part of another study. This thesis will focus on the use of pressure sensitive arrays. Pressure sensitive arrays can be embedded into the environment to provide unobtrusive patient monitoring, using pressure data we can extract clinical information related to the patient's mobility, both static and dynamic. 
Figure 1: Inside the TAFETA apartment [14]
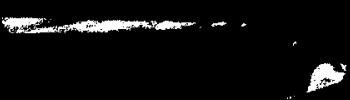

*

Figure 2: Inside the TAFETA apartment; other sensors in use [14] 


\subsection{Mobility and stability}

This thesis looks at unobtrusive patient monitoring using pressure sensor arrays. Patient monitoring is a very generic term; the focus of the thesis is mobility monitoring. Mobility is the ability for the patient to move, this is extremely important for persons living independently. Mobility naturally decreases with age; this can lead to falls which are a significant health concern for older adults [15]. Mobility requires muscle, joint and bone integrity as well as coordination and balance. Older adults need sufficient mobility to perform daily activities independently such as getting into and out of a bed or chair and walking. When the patient performs a bed exit transfer this motion can be captured by a pressure sensor mat installed under the mattress, this can provide lie-to-sit and sit-to-stand transfer times. It is hoped that these transfer times will be useful to help assess the mobility of older adults [16], [17].

The bed exits are performed everyday making this a feasible feature to extract on a regular basis. This allows us to track the patient's mobility over time and detect any abnormalities which could help prevent falls by early intervention. This could also be used to track a patient's recovery in a rehabilitation process. Another important measure of mobility is the person's ability to stand and walk. This thesis also looks at assessing a person's standing stability using pressure sensor arrays placed on the floor. When the patient stands on the sensor array we can extract information related to their static and dynamic mobility. When they stand still we can observe the symmetry in their stance and their area of sway when trying to remain balanced, these measures can help assess their static standing stability [18], [19]. To assess the dynamic standing stability the patient can shift their weight while leaving their feet stationary; this can measure their movement 
velocity and the maximum area of sway [20], [21], [22]. These measures can help assess their mobility limits. When used as part of a rehabilitation program, improvement trends in their standing stability may be found.

\subsection{Rehabilitation}

Rehabilitation is the area of medicine that that helps patients with physical impairments or disabilities regain body function and thus restore their quality of life. Impairments can be related to muscles and tissue, to bones or nerves [23], [24]. Regardless of impairment, it is important for rehabilitation clinicians to develop a client specific treatment plan. Using the processing algorithms with the pressure sensor data, the extracted clinical information can help assess the patient's mobility and stability which can be used to tailor the rehabilitation program. After the rehabilitation plan has been made, it is essential to track progress. Traditionally this is done by various clinicians getting together at weekly team rounds and comparing the results of measures applied occasionally and in artificial situations. Generally, these clinical measures are performed by clinicians without the use of pressure sensor technology [25]. We believe the technology could be used to help assess the patient, providing continuous data on rehabilitation progress. This would allow more precise monitoring and allow earlier intervention if there is lack of progress towards the outcome goals. 


\subsection{Technologies}

Research in patient mobility measurements is not a new field and many different technologies have been tested over the years. Alternative systems for measuring standing stability include force plate systems, accelerometers, pulley systems, and 3D motion tracking which uses cameras with tracking markers attached to the patient's body [26], [27].

Force plate systems use strain gauges at each corner of the plate to measure force and moment in each direction; $x, y, z$. Using these measures the center of pressure (COP) and velocity of COP can be calculated. These measurements can be used to assess the patient's standing stability. Hewson et al. have shown this system can be used to classify elderly persons as fallers and nonfallers [28]. Force plate systems have high accuracy however they are very expensive and are not portable. They also require specially trained personnel to operate.

Triaxial accelerometers measure motion in all three axes; x, y, z. Accelerometers are very low cost and can be integrated into portable equipment. Such a system does not hinder patient movements and these devices have shown to be very useful in detecting falls [29]. There have been many studies using accelerometers to extract other clinical information. Mayagoitia et al. used a single accelerometer attached to the center of the back of the subject and simultaneously recorded data using a force plate system as the subject performed activities to evaluate their standing stability. The accelerometer data were compared to the force plate data and the results showed there was some statistical significance between the measurements [30]. There is potential in using accelerometers to evaluate standing stability however further research needs to be conducted. 
Force plates, pulley systems and 3D motion tracking systems are complex and cumbersome. They cannot be quickly deployed and cannot be used by patients with certain accessibility needs. The pressure sensitive floor tile is a firm surface that does not shift when stood on. Its large area does not hinder the patient's movements. The device is simple to operate and does not require specialized training. As well the pressure sensor array has high accuracy, especially for detecting subtle changes in pressure. The ability to measure subtle chest movements to calculate breathing rates and monitor sleep apnea was shown using bed mat pressure sensors [31], [32].

\subsection{Pressure sensors}

This thesis uses pressure sensor technology exclusively for recording patient data. Pressure readings can be made using different types of pressure transducers; this section will review the types of pressure sensors available. Three types of pressure sensors will be discussed; resistive, capacitive, and fiber optic.

Resistive pressure sensors are composed of two layers of substrate with a conductive core. When pressure is applied the substrates become closer together compressing the conductive core, this decreases the resistance. The change in pressure can be measured by the change in resistance or voltage through the circuit using the resistive pressure sensor. This type of sensor has been used in bed monitoring systems [33] however the main disadvantage is that they are unreliable due the fact that their sensitivity may change over time [34]. 
Capacitive pressure sensors are similar to the resistive type; they are composed of two conductive plates with a dielectric between them. When pressure is applied the plates move closer together, which increases the capacitance. The voltage across the capacitor sensor can be measured and changes in pressure can be measured. The main problem of using capacitive pressure sensors is that when compressed, the dielectric material may not regain its original form quickly, which causes problems of hysteresis and non-linearity [34].

Fiber optic pressure sensors are composed of two optical fibers inserted into a compressible material such as polyurethane foam. One fiber connects to a light source such as an LED, while the other connects to a photodiode. When pressure is applied, the foam compresses which changes the light intensity detected by the photodiode. Similar to the other sensors, the voltage across the photodiode will vary with the pressure applied allowing measurements to be made. Fiber optic pressure sensors have the advantage of being insensitive to $60 \mathrm{~Hz}$ noise and will not be damaged from excessive loading [35]. However the compressible material may be affected by environmental conditions such as temperature or humidity making them softer. The material may not regain its original shape after compression creating problems of hysteresis.

\subsection{Related work}

Carleton University has been a major researcher in patient monitoring using pressure sensors over the years. Theses by Megan Howell-Jones (2006), Idana Veledar (2009), Amaya Arcelus 
(2010), Nicholas Foubert (2010), and Daphne Townsend (2012) [39] have all used pressure sensor arrays to capture data related to patient monitoring.

M. Howell-Jones used bed based pressure sensors with a $10 \mathrm{~Hz}$ sampling rate and captured short term recordings from participants. She developed segmentation algorithms to identify participant movements, and extract breathing signals from segmented non-movement data [40].

I. Veledar used bed based pressure sensors with a $10 \mathrm{~Hz}$ sampling rate to capture short term recordings from participants at the Élisabeth Bruyère Research Institute. She developed algorithms to extract hip and hand regions from the recordings. She was able to extract sit-tostand times using a pressure sensor sum [41].

A. Arcelus used bed based and floor tile pressure sensors in a smart home environment to capture short term recordings from participants. She developed algorithms to extract sit-to-stand times, and detect hand use during the transfer. She looked at center of pressure analysis from the bed sensor. She also investigated the use of these sensors in smart home monitoring and mobility monitoring [42].

N. Foubert used the same pressure sensors and recordings from Bruyère as Veledar, as well as a simulated activity recording. He developed algorithms to recognize if a person is sitting, lying in a particular posture, or transitioning from lying to sitting [43].

This thesis is different from the previous work, in that we use a floor tile based pressure sensor to extract clinical information related to standing stability. It uses a novel processing algorithm, and real patient data collected at Élisabeth Bruyère Research Institute. We also use a new type of bed based pressure sensor which uses a $20 \mathrm{~Hz}$ sampling rate with a different layout of sensors. Long 
term data was collected from participants in their own home and in their own bed. This introduced a new problem, in which different mattresses would be placed on top of the sensor, and participants were not limited in their bed exits. We recorded natural unobtrusive data in a real world environment. The long term recordings introduced a need for a robust algorithm that could extract clinical information from 100 s of hours of recordings. We believe this work represents real world application of patient monitoring using pressure sensor arrays. The algorithms developed are novel and best suited for this situation. 


\section{Chapter 3: Experiment Setup}

\subsection{Introduction}

Chapter 3 deals with the experimental setup for collecting data required for this thesis. There are two different pressure sensor arrays used in this project, a floor tile based unit and an under mattress unit. Section 3.2 will deal with the floor tile sensor, the hardware and equipment setup will be discussed and followed by the data recording procedure. Section 3.3 will deal with the under mattress or bed sensor, again the hardware and equipment setup will be discussed, followed by the data collection procedure.

\subsection{Floor sensor}

This section discusses the experiment setup using the floor tile sensor, the hardware and equipment setup will be discussed, followed by the data recording procedure. 


\subsubsection{Hardware}

To measure the patient's standing stability an algorithm to monitor their subtle movements in stance was developed. The technology used was an array of pressure sensors built into a $61 \mathrm{~cm} \mathrm{x}$ $61 \mathrm{~cm}$ floor tile. The device (HRFP022) was developed by Tactex Controls, Inc. in BC, Canada. The floor tile is shown in Figure 3. The device uses "Kinotex RST" proprietary sensing technology to measure change in force and pressure distribution. The Kinotex sensors are fiber optic pressure sensors. It uses a light source to shine light into a foam sensing surface, when pressure is applied to the surface, the light scatters within the foam. The change in density of the backscattered light is measured [44]. The measured density is directly related to the pressure applied to the sensor.

This model used an array of $48 \times 48$ pressure sensors, for a total of 2304 sensors. This creates a high spatial resolution array, with a center to center sensing point density of $12.7 \mathrm{~mm}$ [44]. Pressure sensitive technology has gained popularity in medical research as it is non-invasive and unobtrusive to patients. With multiple sensors composing a sensor array the system can detect very subtle changes in sensor pressure which has been used in the past to extract clinical information such as sit-to-stand time in order to quantify a patient's mobility [36]. The floor sensor pressure array is also a non-invasive device which is ideal for monitoring the patient's rehabilitation. 


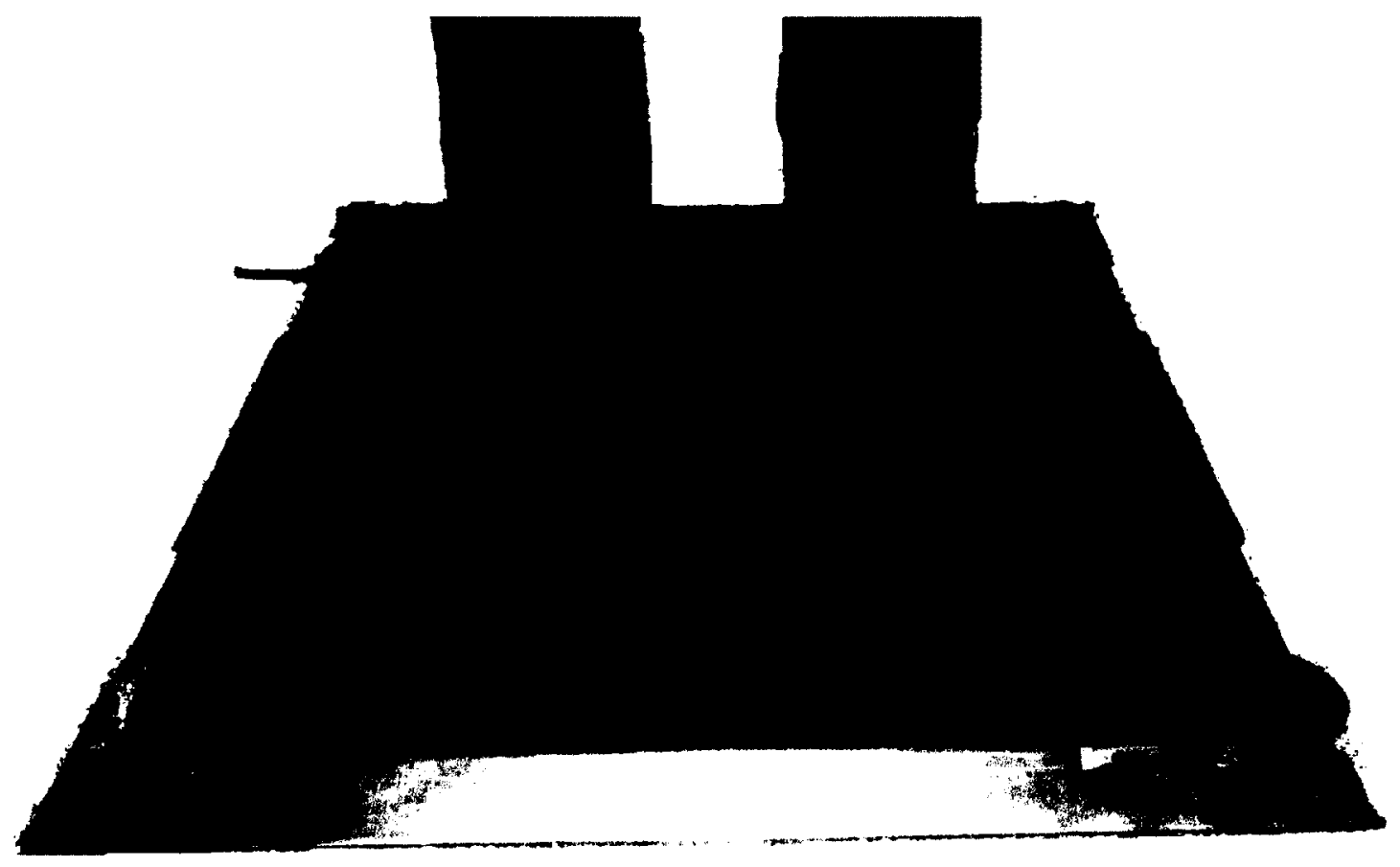

Figure 3: Floor tile pressure sensor with person standing on it

\subsubsection{Equipment setup}

The floor tile sensor was placed on the ground with plenty of space around it to prevent injury when the participant stands on it. The sensor is connected to an ADC converter box using a specialized cable. The box is then connected to a laptop running the Tactex capture software via a USB connection. The ADC unit and laptop connections are shown in Figure 4. The recording software interface is shown in Figure 5. 

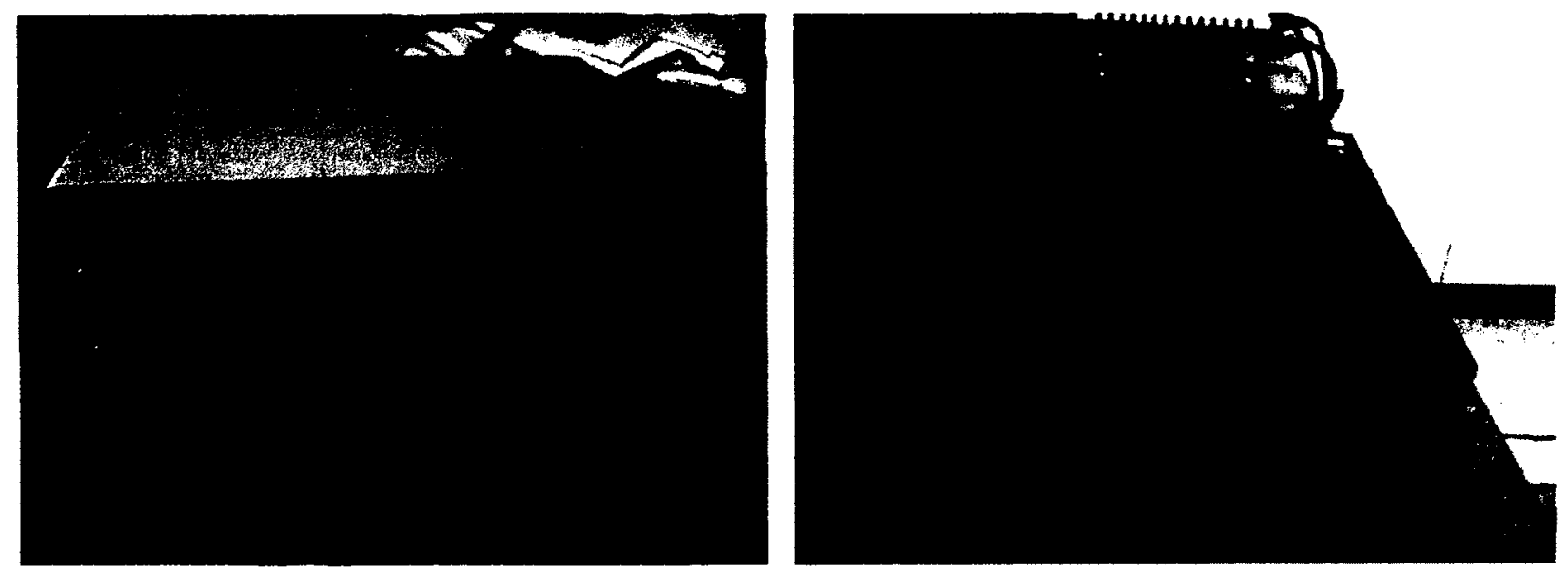

Figure 4: Equipment setup for floor tile pressure sensor

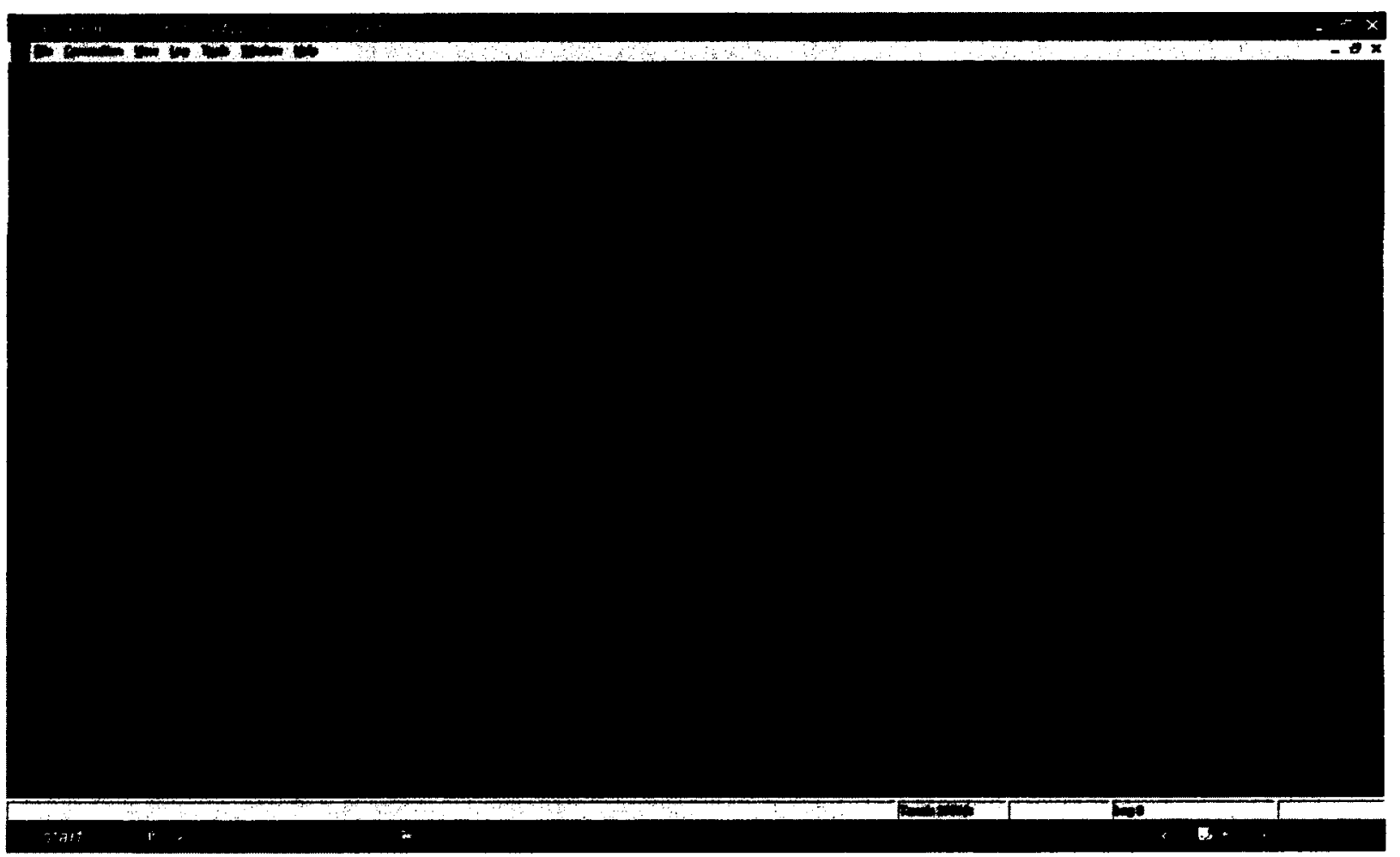

Figure 5: Tactex data capture interface for floor tile pressure sensor 


\subsubsection{Data format}

The floor sensor records changes in pressure applied to the individual sensors at a sampling rate of $50 \mathrm{~Hz}$. The software only logs values for sensors that have observed a change in pressure from their previous value. The data were recorded into a CSV file which was processed by the stability measuring algorithm developed in MATLAB (MathWorks, MA, USA). An example of recorded data is shown in Table 1 . There is a brief header specifying the file name and location and the time the file was created. The first column, labelled tick, is the sample number. The second column is the time at which the sample was taken. The third column, labelled event, is triggered by an input on the capture software. When the operator clicks "Event", an incremental number is placed in this column. The fourth and fifth columns are the most important. TxlIndex column refers to which of the 2304 sensors has been sampled; the pressure reading of that sensor is placed in the fifth column, TxIValue. The processing algorithm maps the pressure readings to their geographic location.

Table 1: Floor sensor data example

\begin{tabular}{|c|c|c|c|c|}
\hline \multicolumn{5}{|c|}{ 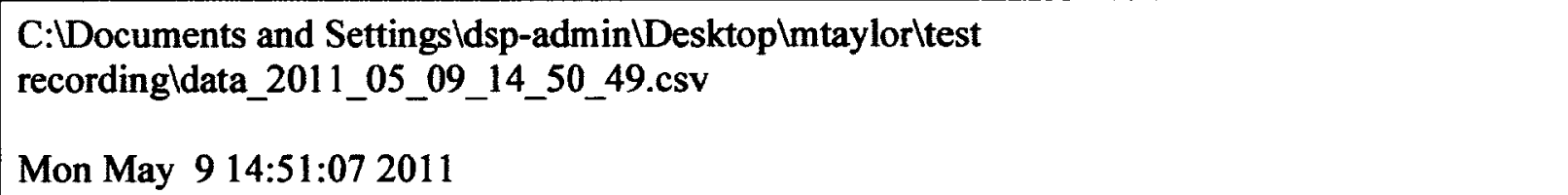 } \\
\hline Tick & hr:min:sec:ms & Event & Txllndex & TxIValue \\
\hline 448832110 & $14: 51: 7: 437$ & 0 & 615 & 476 \\
\hline 448832110 & $14: 51: 7: 437$ & 0 & 621 & 638 \\
\hline 448832110 & $14: 51: 7: 437$ & 0 & 694 & 622 \\
\hline 448832110 & $14: 51: 7: 437$ & 0 & 67 & 506 \\
\hline 448832110 & $14: 51: 7: 437$ & 0 & 1163 & 440 \\
\hline
\end{tabular}




\subsubsection{Data collection}

We collected standing stability data from two subjects. The first was an older adult who was participating in the Bruyère Stroke Rehabilitation program, and the second was a healthy younger adult. The floor sensor was installed at the Bruyère Continuing Care facility and data were collected as part of an ongoing clinical intervention trial. Ten 60 -second recordings were made of quiet standing and lateral weight shifting over a two week period.

\subsection{Bed sensor}

Section 3.3 will deal with the under mattress or bed sensor, again the hardware and equipment setup will be discussed, followed by the data collection procedure.

\subsubsection{Hardware}

To assess the patient's mobility using the bed exit and entry times, pressure sensitive technology was installed under the mattress of the patient's bed. The sensor was developed by S4 Sensors Inc (formerly known as Tactex Controls Inc.) in Victoria, BC, Canada. It is in the form of a three sectioned mat of size 34 " $\times 34$ " $(86 \mathrm{~cm} \times 86 \mathrm{~cm})$. Each section contains an array of $3 \times 8$ pressure sensors. The three sections compose the overall mat which contains 72 pressure sensors in a $9 x$ 8 array. The pressure mat is shown in Figure 6. The sensors are placed equal distance apart in a grid formation, this is an improvement from previous honeycomb tessellation in terms of signal 
processing ease. The sensors are spaced approximately $9 \mathrm{~cm}$ from each other horizontally and vertically, this spacing is consistent across the foldable sections of the mat. Figure 7 is an illustration showing the sensor layout. The sensors are sampled at a rate of $20 \mathrm{~Hz}$, an improvement from the older sensors mats with $10 \mathrm{~Hz}$ sampling.

The pressure sensors used are fiber optic and connect to a control circuit embedded in the mat. There is one control circuit for each of the three sections; these are illustrated by the green and brown units in Figure 7. The sensors measure pressure by measuring the amount of reflected light passing through a foam material which is compressed when the patient sits on the mat. It measures the values in relative units and not in Newtons or pounds. It measures pressure within a range of $0-3072$.

The mat connects to a Bluetooth transmitter via a cat 5 cable. The Bluetooth transmitter, shown in Figure 8 is paired to a Bluetooth device to receive serial data transmission; in our case we used a Dell Optiplex PC with an ASUS Bluetooth adapter. 


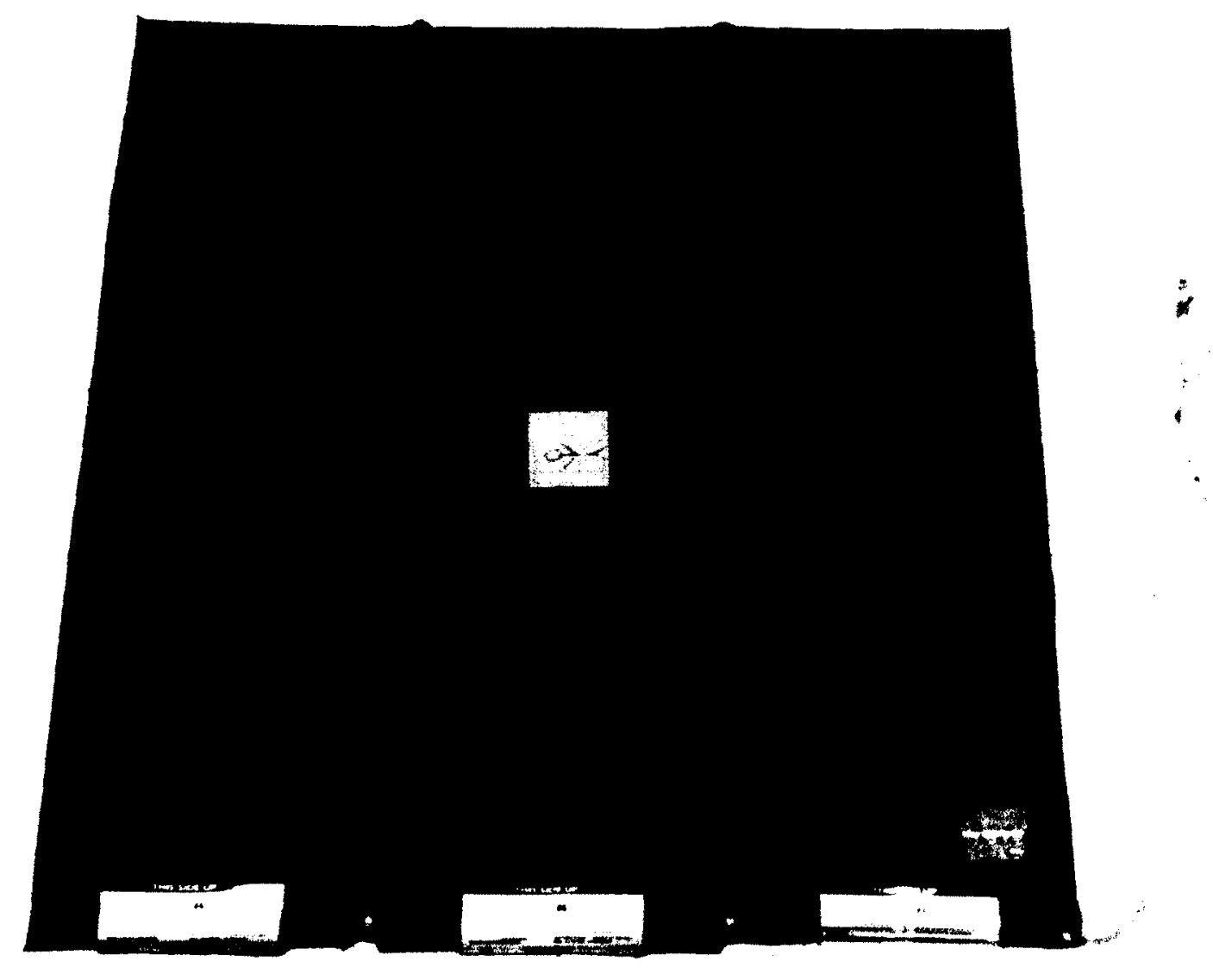

Figure 6: Bed mat pressure sensor array 


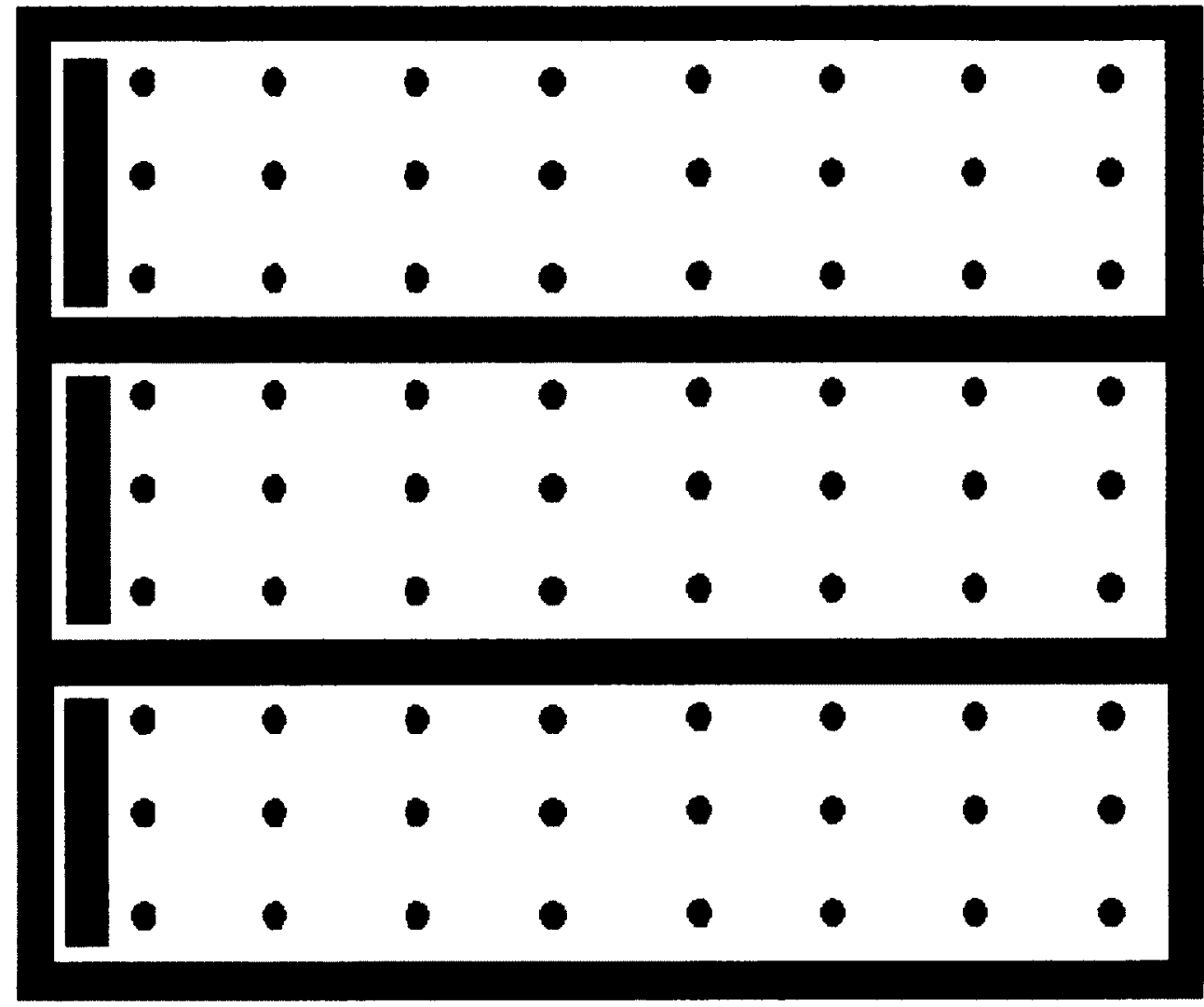

Figure 7: Illustrated layout of individual sensors (red) in the bed pressure mat. Circuit board is shown in green.

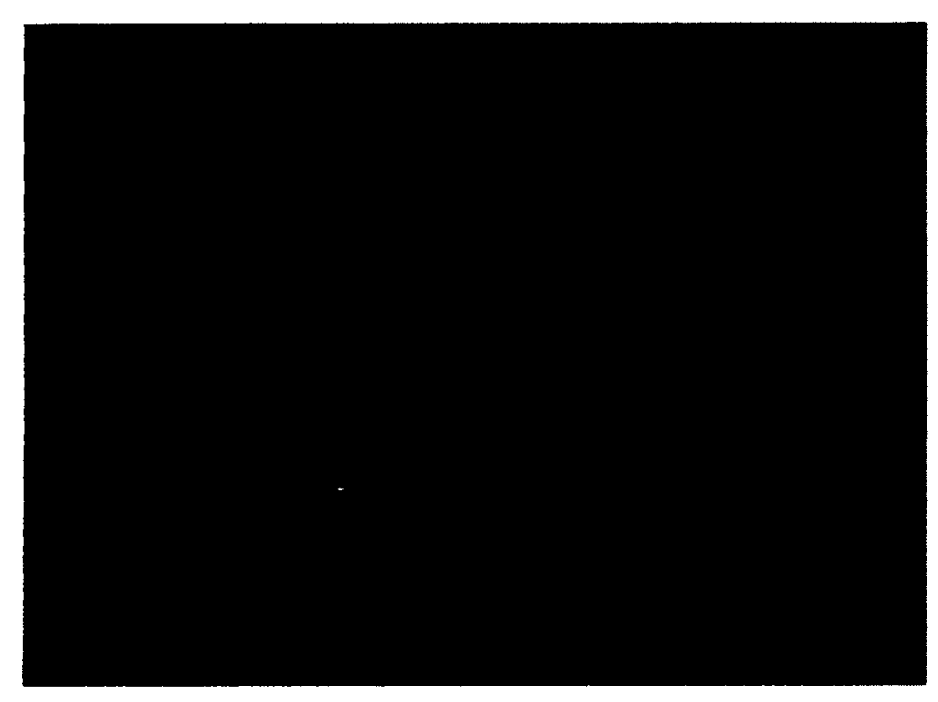

Figure 8: Bluetooth transmitter unit for bed mat sensor 


\subsubsection{Equipment setup}

The pressure sensitive mat is installed between the patient's mattress and the bed frame or box spring. In order to take proper pressure measurements the foam within the sensor must be compressed, therefore the sensor must be placed on a solid based to provide reaction force. To ensure a consistent surface a piece of $1 / 4 "(0.64 \mathrm{~cm})$ plywood of size 36 " x 36 " $(91 \mathrm{~cm}$ x $91 \mathrm{~cm})$ was placed on top of the box spring, with the pressure sensor taped to it to prevent it from sliding. The cable is run along the side of the mat and to either the head or foot of the bed to be as unobtrusive as possible and prevent a tripping hazard. The pressure sensor is orientated the same in every participant's home with the top of the mat placed at the head of the bed. The sensor is positioned so the edge lines up with the edge of the bed that the participant usually exits from; it is centered in spot where the participant usually sits before standing up to exit the bed. This is the optimal position for capturing as much information possible related to the transfer. Figure 9 illustrates where the pressure sensor is installed in the scenarios of a small or large bed. Figure 10 is side view showing how the pressure sensor is placed between the box spring and the mattress with all the other equipment placed underneath the bed frame. 


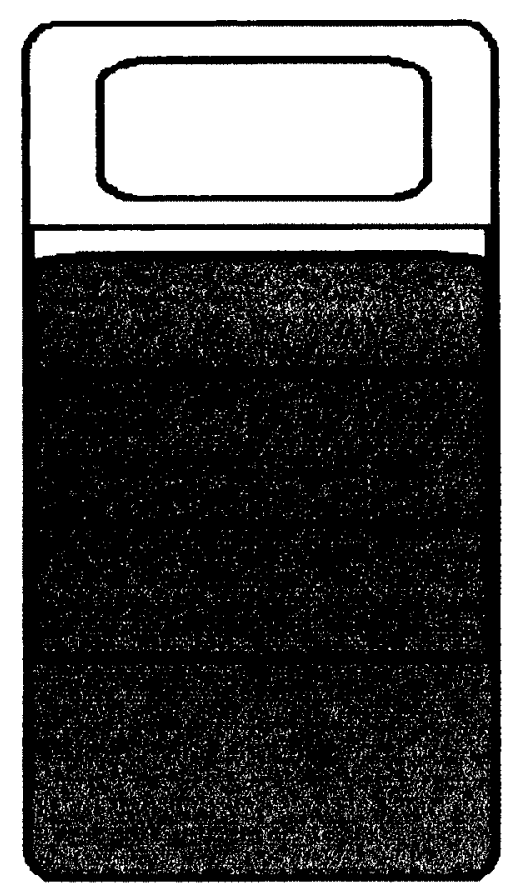

Small bed

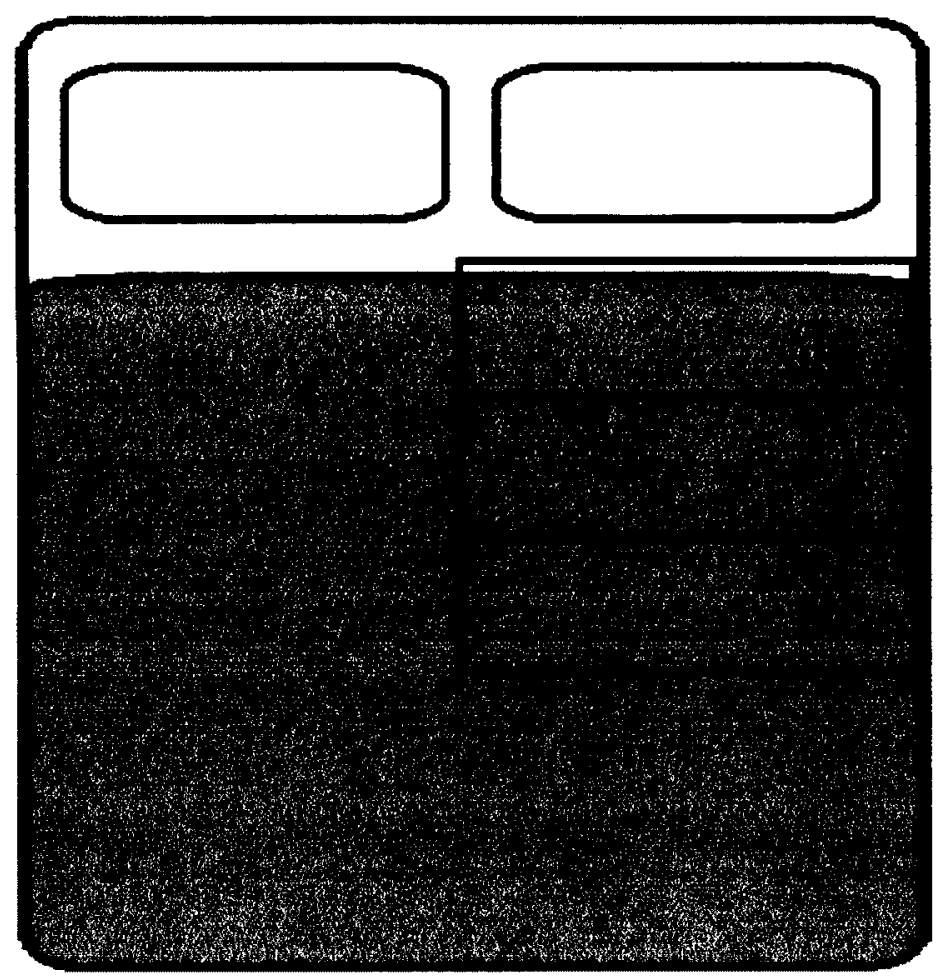

Large bed

Figure 9: Pressure mat orientation under mattress. Pressure sensor is in blue and shows the three sections of the array.

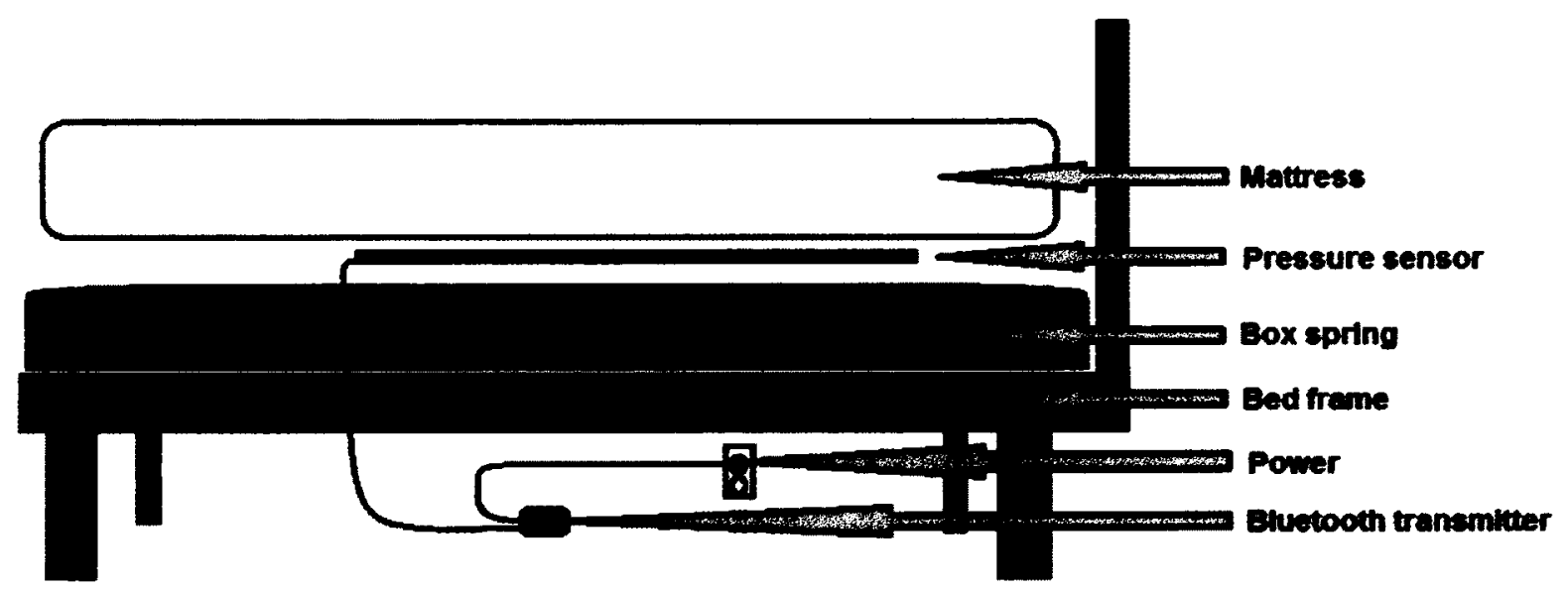

Figure 10: Side view of equipment install under mattress. 
The Cat 5 cable from the pressure mat is ran underneath the bed and connects to the Bluetooth transmitter unit. The Dell Optiplex PC can be placed underneath the bed or in a closet nearby. The Bluetooth transmitter and $\mathrm{PC}$ are connected to a power bar with surge protection which is connected to the mains. There are no peripherals or monitor connected to the PC when the recording setup is left in the participant's home.

The PC runs a custom install of Windows XP. Each computer is outfitted with a 500 GB hard drive, the usable data recording size is $460 \mathrm{~GB}$. The Windows XP install is barebones, all Windows update features, screen savers, power saving modes, internet connections are disabled. The $\mathrm{PC}$ is configured to automatically boot on $\mathrm{AC}$ power, to ensure recording will resume in case of a power outage. The PC is linked to a specific Bluetooth transmitter unit and will not accept connections from any other Bluetooth devices. S4 sensors capture software is automatically run on start-up and it searches for the Bluetooth transmitter unit previously linked, once the connection is established recording begins immediately. The pressure data comes in ASCII format and is recorded into CSV files and placed in a specified folder on the PC. A new file is created every hour. Each hour long recording requires approximately $20 \mathrm{MB}$ of space; 480 MB for 24 hours. The $460 \mathrm{~GB}$ usable space will allow for approximately 981 days of continuous recording. This is more than adequate as the longest any one of these systems will be installed is 1 year.

$460 \mathrm{~GB}=471040 \mathrm{MB}$

$471040 \mathrm{MB} / 480 \mathrm{MB} /$ day $=981$ days 


\subsubsection{Data format}

Data logging is controlled by the S4 Sensors software. The software has been configured to begin recording pressure readings automatically once the Bluetooth connection to the mat has been established. Data is logged in ASCII format CSV files on the hard drive. A new file is generated every hour. Every file has a header specifying the file location and the time it was created. The reading from each of the 72 sensors is placed into column. A new row of readings is created every 0.05 seconds. Table 2 is an excerpt from a recording showing only 4 samples for 4 sensors.

Table 2: Bed sensor data format example

E:IS4 Sensorsidata_sn4358_2012_02_21_13_15_01.csv

Tue Feb 21 13:15:01 2012

\begin{tabular}{|l|l|l|l|l|l|l|l|l|}
\hline TimeStamp & Sample & Event & pressure (mPSI) & $\mathbf{t x 1 1}$ & $\mathbf{t x 1 2}$ & $\ldots$ & $\mathbf{t x 1 7 1}$ & $\mathbf{t x 1 7 2}$ \\
\hline 13.15 .01 .390 & 257 & 0 & 0 & 241 & 279 & $\ldots$ & 334 & 256 \\
\hline 13.15 .01 .453 & 258 & 0 & 0 & 242 & 280 & $\ldots$ & 335 & 256 \\
\hline 13.15 .01 .484 & 259 & 0 & 0 & 241 & 279 & $\ldots$ & 335 & 256 \\
\hline 13.15 .01 .531 & 260 & 0 & 0 & 241 & 278 & $\ldots$ & 334 & 256 \\
\hline
\end{tabular}

The first column is the timestamp; the second column is the sequential sample number, the third column is used by the operator to mark an event in the recording, a key must be pressed to trigger the event, which places an incremental number in the event column. The forth column is unused. The remaining columns, 5 to 76 are the ADC values from the individual pressure sensors. In this example, the sensor readings are under 350 , therefore the pressure mat is currently unloaded.

The timestamp and the sensor values are the most important columns of data, we use the timestamp to determine the exact timing of important events in the recording. The sensor values 
are mapped to their geographical location to generate images of the pressures observed by the mat.

\subsubsection{Data collection}

Data was collected from multiple participants in their homes. The Bruyère Research Institute recruited participants living in subsidized seniors' apartments in the Ottawa area to partake in the study. With the participant's consent, pressure sensitive mats were installed underneath their mattress and pressure data was recorded. The participants agreed to allow us to record pressure for up to 6 months continuously. The participants varied in ages and mobility from 60 s and very mobile to 90 s and very frail.

Our colleagues at Bruyère would visit the participants on a monthly basis to perform clinical measures and evaluate their mobility. This would include a video recorded session in which the participant would lie in their bed and perform normal exits. At each visit they would perform three bed exits; this includes lie-to-sit and sit-to-stand. At each visit the data would be transferred to a secure portable hard drive.

Using the video recordings the pressure data could be analyzed and features could be found to classify the position the person was in, lying, sitting, or transitioning. As well the accuracy of the lie-to-sit and sit-to-stand measuring algorithms could be evaluated. 


\section{Chapter 4: Processing Algorithms}

\subsection{Floor sensor}

Chapter 4 discusses one of the engineering contributions from this thesis; the novel processing algorithms. Section 4.1 is entirely for the floor tile based pressure sensor array. Design considerations are made which to lead to the development of a novel processing algorithm to meet the objectives of the thesis and requirements of the researchers.

\subsubsection{Generating pressure map images}

The algorithm determines the patient's center of pressure using image processing techniques. The CSV file containing the raw pressure data were used to generate images representing the pressure seen on the floor sensor. The pressure sensor array samples at a maximum rate of $\mathbf{5 0}$ $\mathrm{Hz}$, allowing quick movements to be captured. However when using the device to capture movements from rehab patients, it was observed that high speed $50 \mathrm{~Hz}$ sampling was not necessary and could be considered a waste of resources. To reduce the processing and bandwidth requirements, the raw data were averaged over a $300 \mathrm{~ms}$ time period, thus reducing the effective 
sampling rate to $3.3 \mathrm{~Hz}$ to create images. This essentially reduced the processing load by $15 x$. The images created were $48 \times 48$ pixels where each pixel represents one pressure sensor. The images are in greyscale and therefore can show up to 255 different pressure levels. The raw data were normalized to fit into this range.

An example of the raw data recorded at $50 \mathrm{~Hz}$ and converted into an image is shown in Figure 11. The raw data were then averaged over a $300 \mathrm{~ms}$ window to generate the image shown in Figure 12. The images were stretched from their original size to make them easier to see. The patient's foot prints can be seen in both images in the same position. The image generated by averaging has more noise; however this is removed in the following step.

Figure 11: Pressure mapped image created from $50 \mathrm{~Hz}$ sampling of patient standing on pressure sensor array

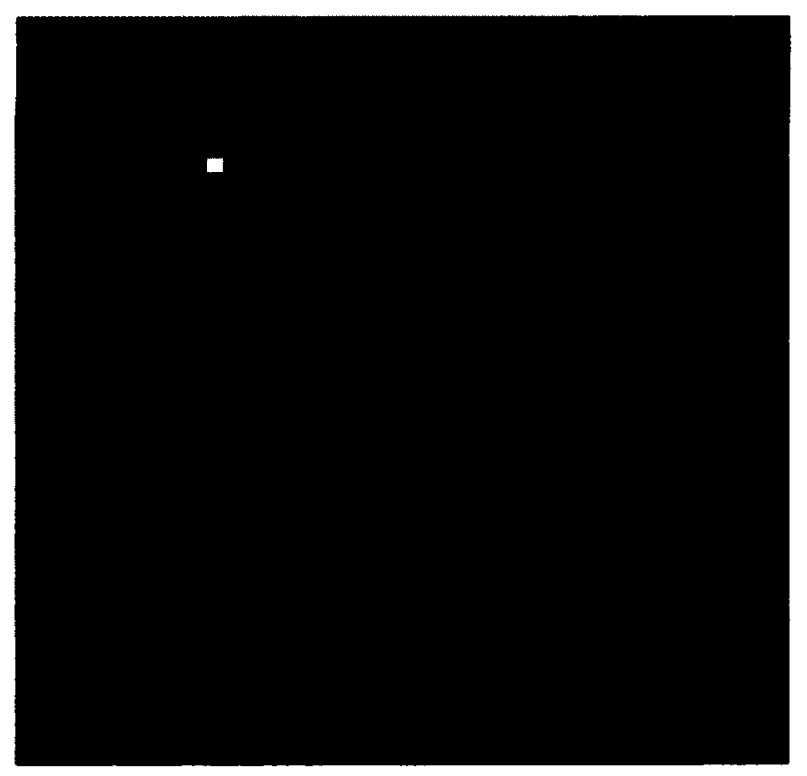

Figure 12: Pressure mapped image created from $300 \mathrm{~ms}$ averaging of patient standing on pressure sensor array 


\section{Noise removal}

The images created after averaging appear to have more noise than the original image, this is because the noisy pixels accumulate. However, the foot pressure signal strength increases at a rate higher than the noise, thus the signal to noise ratio (SNR) increases. This is a benefit of using averaging. Image processing techniques are used to remove the noise from the images and to determine the center of pressure for each foot and for the patient's stance. The processing algorithm is summarized in the flow chart in Figure 13. The algorithm uses the following morphological filtering stages: The image is dilated which enlarges and connects nearby pixel structures. The image is then eroded which shrinks and smoothes the structures. The structural element used in both of these steps was a flat disk shape with a radius of 10 pixels. The final processing technique is used to remove the small pixels with low values that exist because of noise. A threshold is set to eliminate these small pixel structures. 


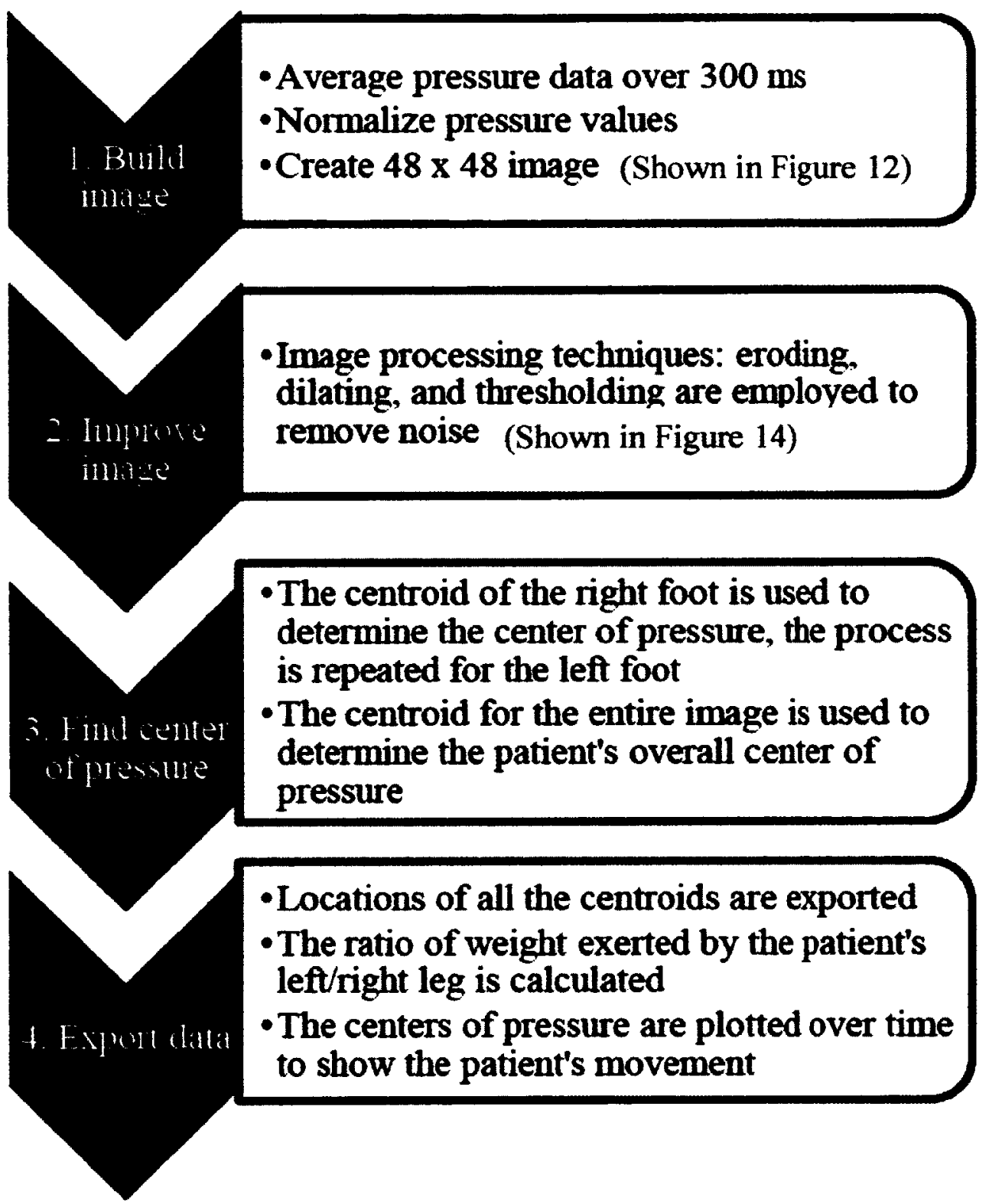

Figure 13: Flow chart for proposed algorithm to measure stability 


\section{Center of pressure}

After the image processing techniques are used on the original image the resulting image shows two very distinct objects which are feet. The intensity-weighted centroid for each foot is calculated by averaging the pixel values, which correspond to pressure levels, to find the point which is the geometric center of the object. The centroid locations are superimposed onto the image and exported. Figure 14 shows this, the COP for the left foot is in green, the right foot in red, and the overall COP in blue. Calculating the centroid of the pressure field is an accurate method for estimating the center of pressure [37].

Further processing using the center of pressure measurements can be done to extract other valuable clinical information such as the COP velocity or the area in which the COP has varied. These measures could help in assessing the patient's standing stability and tracking their recovery during rehabilitation. 


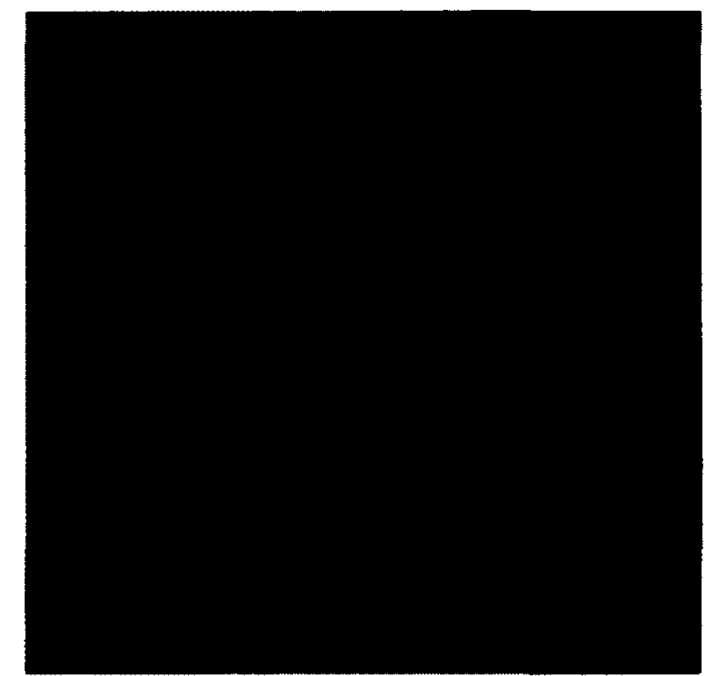

(a) $48 \times 48$ resolution

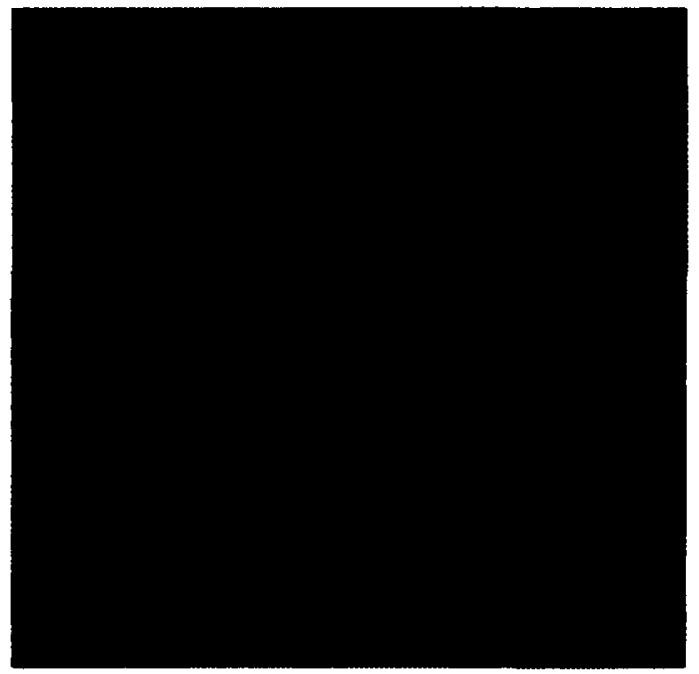

(c) $12 \times 12$ resolution

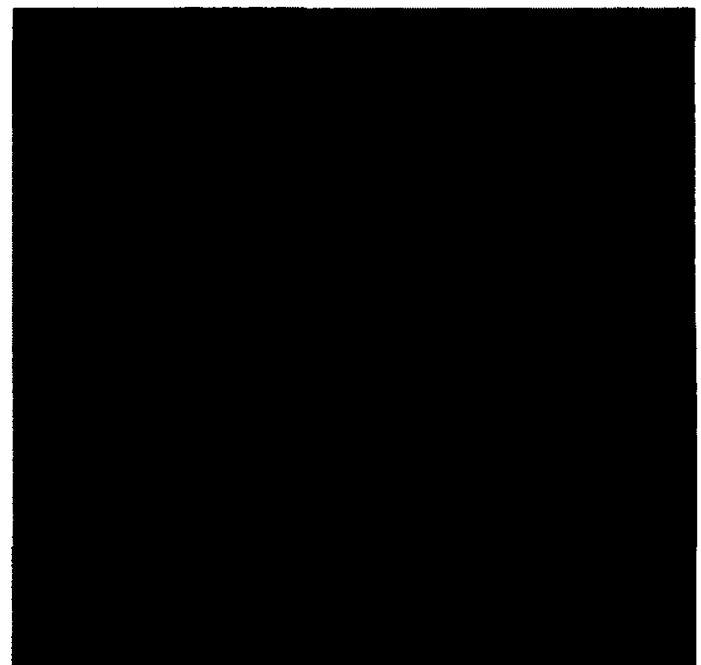

(b) $24 \times 24$ resolution

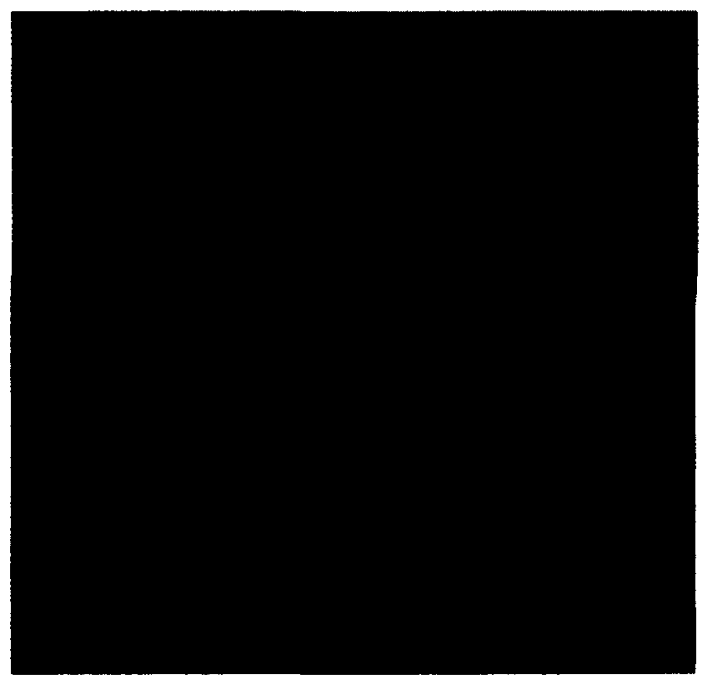

(d) $6 \times 6$ resolution

Figure 14: Processed images of patient on sensor at varying image resolutions with centers of pressure overlaid 


\subsubsection{Extracting clinical features}

The system was designed to provide clinicians with measures of four clinically relevant features that could assist in the assessment of an individual's standing stability. The first feature, we will call the "lean ratio" is how much the person leans and in what direction while standing still. This piece of information allows clinicians to quantify how much the patient is favouring one side of their body due to stroke, hip surgery, a fall, or other ailment. This allows the clinicians to personalize their treatment plan to include strengthening and balance training exercises to rehabilitate the patient to proper standing without any lean. By measuring their lean ratio over time the clinician and patient can track their recovery and make changes to improve recovery times.

The second clinical feature, we will call "footprint symmetry" is how much pressure the patient exerts under each foot and in what regions of the foot. It is ideal that the person has symmetry in weight bearing or an even distribution of pressure under each foot [38]. This can provide information to complement the first clinical feature, as to why the patient favours one side of their body. It could be they require orthopedic inserts to correct asymmetrical standing.

The third clinical feature, we will call "sway area" is how much the patient sways while attempting to stand still. This feature is measured based on the movement of the patient's center of pressure in the $\mathrm{x}$ and $\mathrm{y}$ axis, which corresponds to lateral and forward/backward movement respectively. The sway envelope, or total area in which the center of pressure has varied is also calculated. This feature gives the clinician a measure of the patient's balance and stability. Measuring the center of pressure displacement before, during and after an intervention, the clinician and patient can track changes and make adjustments for optimal outcomes. For 
example, the clinician can recommend the use of a cane or walker to assist patients that have difficulty maintaining static balance.

The fourth clinical feature, we will call "movement velocity" characterizes dynamic balance. The patient is instructed to shift their weight from side to side as quickly as possible. The processing algorithm quantifies the speed at which their center of pressure changes. By measuring this feature over time we can document an individual's ability to control the movement of the body through space and to determine an individual's maximum stability limits. It could also be used to identify trends of decreasing mobility due to impairment or increasing mobility due to, for instance, a training program for the individual patient.

\subsubsection{Design of GUI}

A graphical user interface (GUI) was developed for researchers to analyze pressure recordings from the floor tile. The GUI is shown in Figure 15. The GUI was designed to provide researchers with a simple way to process multiple data recordings quickly. All the available options for processing can be changed easily. The data can be processed using the full $50 \mathrm{~Hz}$ algorithm or the reduced resource $3.3 \mathrm{~Hz}$ algorithm. There are 5 options for exporting the processed data, including visual outputs of pressure mapped images, videos, and COP plot videos. Data values can also be exported to CSV files; this includes the COP locations, and the clinical features. 


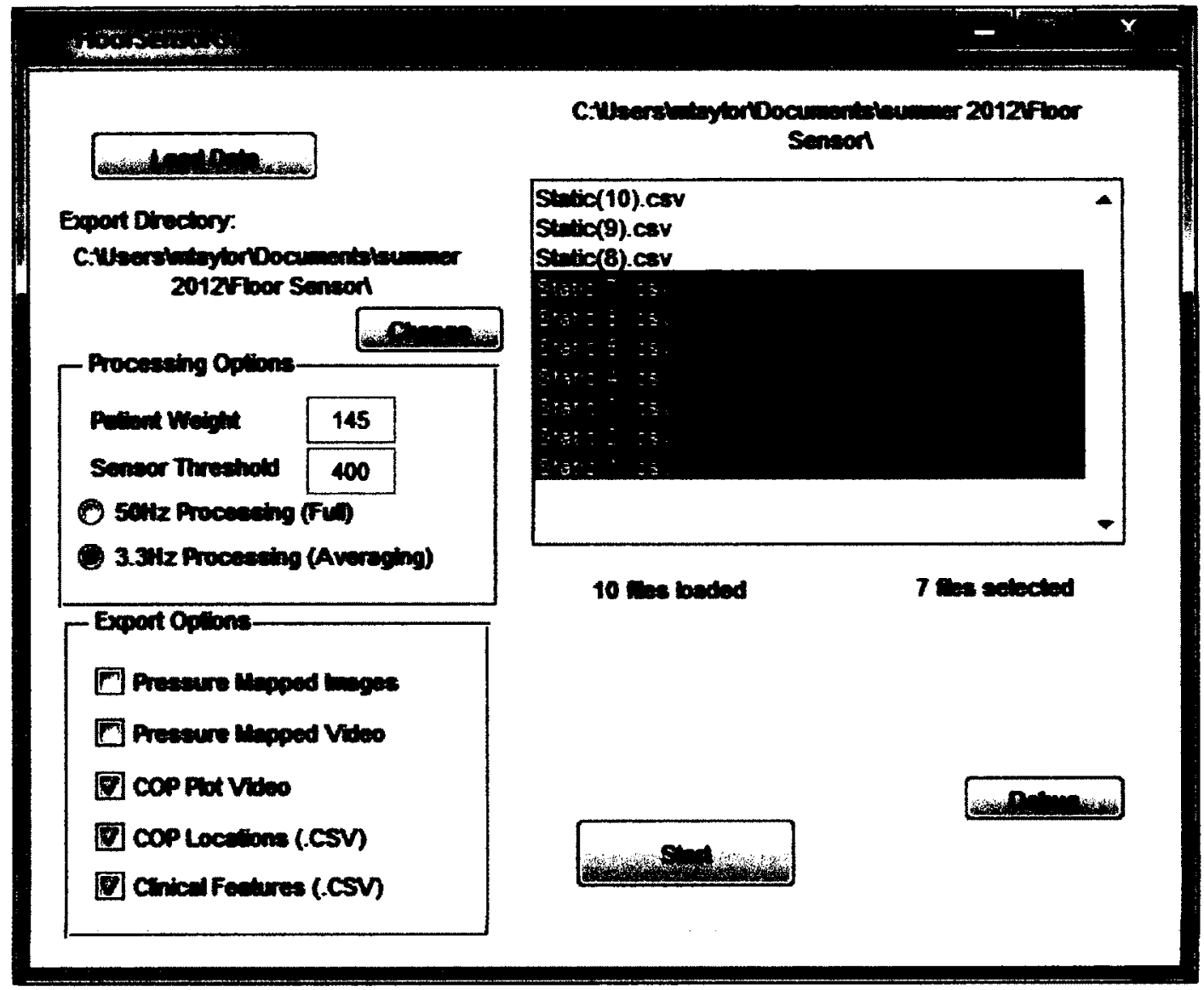

Figure 15: Floor Sensor GUI 


\subsection{Bed sensor}

This section, 4.2 is entirely for the bed mat based pressure sensor array. A novel processing algorithm, one of the engineering contributions from this thesis is discussed. Design considerations are made which lead to the development of a processing algorithm to meet the objectives of the thesis and requirements of the researchers.

\subsubsection{Signal feature extraction}

To extract clinically relevant information the raw pressure data must be processed into a form that can be analyzed easily and reliably. The simplest form of analyzing the data was to look at the pressure reading values as they changed over time, however with 72 sensors, the best way to observe all of them were to sum the pressure values for each sample. We call this the pressure sum (PrS). The equation for the pressure sum is defined in Equation 1 and is the summation of the values for sensors 1 to 72 at that sample (t).

Equation 1: Pressure Sum

$$
\operatorname{PrS}(t)=\sum_{i=1}^{72} s_{i}(t)
$$

The pressure sum for an entire recording can be processed in a relatively quick amount of time as no complex mathematics is involved. However there is a limited amount of information that can be extracted from this feature. It is the best feature for determining bed occupancy. When the 
person is not in the bed, we say the sensor is unloaded, there is a static value for PrS. Depending on which pressure sensor array was used and what mattress is placed on top of it, the static unloaded pressure sum is usually between 18000 and 19500 .

The pressure sum increases rapidly when a person enters the bed, or the capture region of the sensor array. Using the pressure sum it is easy to say the person is either sitting, lying, kneeling, or standing on the bed, however it is almost impossible to discern which of these states it is using PrS alone. This is because we only have a measure of total pressure, it would be expected that if the person was sitting on the bed, with their entire weight within the region of the sensor, this would be the same $\operatorname{PrS}$ as if they were lying on the bed, with their entire weight within the sensor field. The PrS range when loaded varies based the weight of the participant; we have seen values from 24000 to 60000 .

When the person exits the bed they would place their feet on the floor and sit on the edge of the bed, we would expect the PrS to be less in this case as some portion of the person's weight is outside of the pressure sensor field. Therefore we could use this to discern between sitting and lying states, however this is not the case. The sensor field is a square of 34 " $\mathrm{x} 34$ " and when a person of average size is lying in the bed some of their weight, be it their head or legs, is outside the field of capture. This lowers the PrS value and in many cases it was impossible to discern between sitting and lying using $\operatorname{PrS}$ alone because the values are so close.

\section{Pressure concentration}

To better determine if the person is sitting or lying we needed to examine the concentration of pressure. It's expected that when the person is lying their pressure is spread across a larger area, 
more disperse, compared to when they are sitting on the edge where it is entirely concentrated. In order to examine the pressure concentration we needed to convert the pressure values from columns into more visual representations, we needed to create pressure mapped images.

\section{Pressure mapped images}

To convert the data into images, a grid matrix needed to be created where each sensor number was placed in its correct geographic location. A $9 \times 8$ matrix was created in MATLAB with empty values. For each sample, a new matrix was loaded and all 72 pressure readings were placed in their correct location. The pressure readings are represented by ADC values and can vary between 0 and 3072; therefore it was necessary to normalize the values into the range of 0 to 255 to be able to convert them into greyscale images.

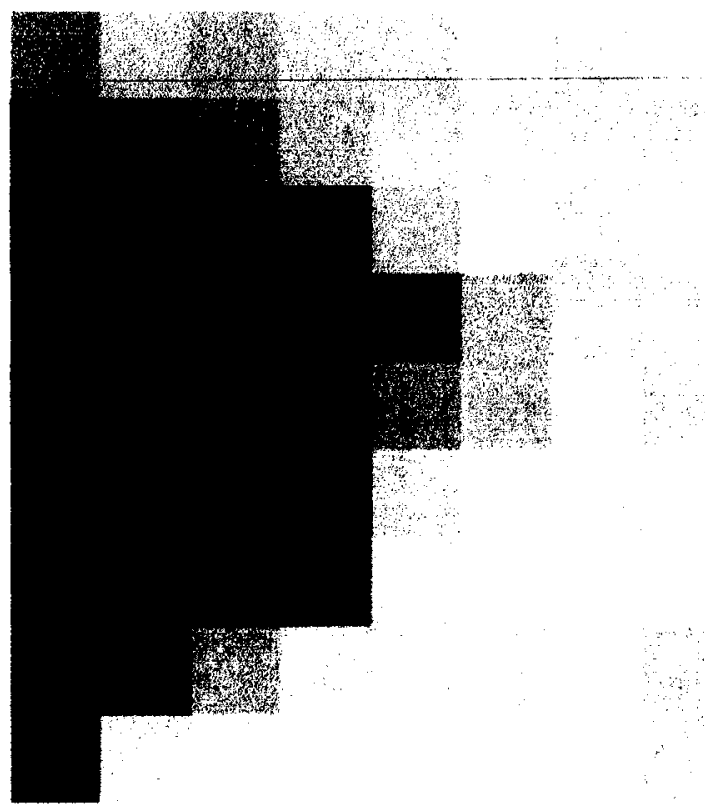

Figure 16: Pressure mapped image showing concentrated pressure (person sitting)

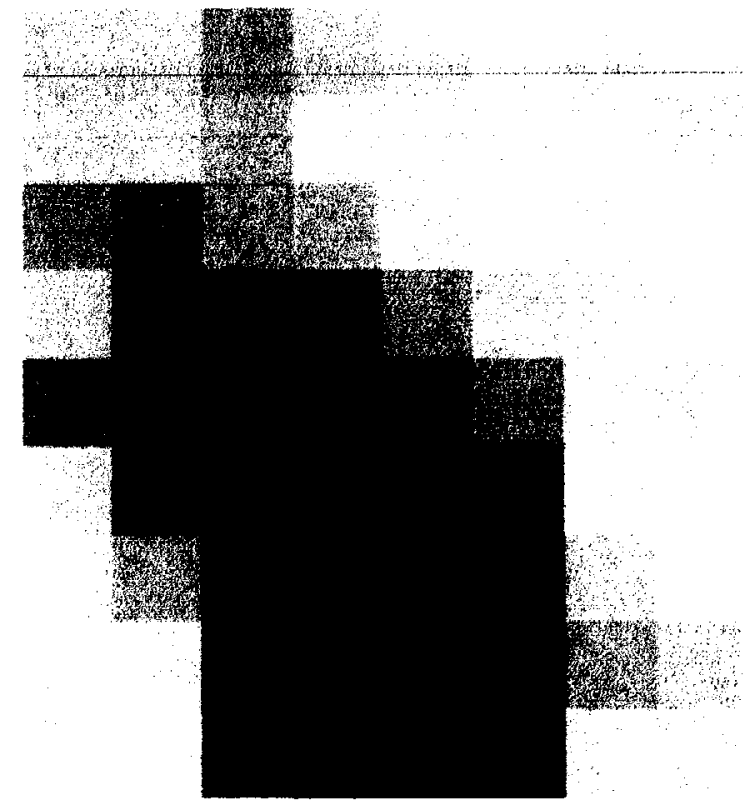

Figure 17: Pressure mapped image showing dispersion (person lying) 
By observing the pressure mapped images manually we could easily tell the difference between concentrated pressure near the edge which would indicate the person was sitting, as shown in

Figure 16 and when the pressure was disperse across the vertical field when they were lying down, as shown in Figure 17. This characteristic was easily discovered with the human eye however it needed to be detected by software in order to automate the clinical information extraction process. The first method of quantitatively analyzing the pressure concentration was by counting the number of active sensors.

The first step was to determine whether the sensor was active or not, a fixed threshold would have been a simple solution, however we found that when unloaded the sensors did not have a fixed reading. The unloaded readings varied from 150 to 350 , we could not use an average between either as that would cause us to count some of the sensors as active all the time. We also could not use a threshold of 350 because in some instances when light pressure was applied, as would be seen under a hand, to one of the sensors with a low unloaded reading, that light pressure may only generate a reading of 300 which would be below the threshold. The best solution was to calculate the average value of each sensor in the unloaded state over a 1 second. The equation to calculate the average unloaded value is shown below, where $i$ is the individual sensor and must be calculated for each of the 72 sensors, $t$ is the sample and the average is calculated using 20 samples ( 1 second at $20 \mathrm{~Hz}$ ).

\section{Equation 2: Sensor average}

$$
\operatorname{savg}_{i}=\left(\sum_{t=1}^{20} s_{i}(t)\right) / 20
$$


With a threshold determined for each of the 72 sensors we could then look at the recording and determine which sensors are active by simply seeing if the reading is greater than the threshold. The software increments a counter every time a sensor is greater than the threshold, this is expressed mathematically below.

Equation 3: Number of Active Sensors

$$
\begin{gathered}
\text { NumActive }=\sum_{i=1}^{72} \operatorname{Active}\left(s_{i}(t)\right) \\
\text { Where } \operatorname{Active}\left(s_{i}(t)\right)=\left\{\begin{array}{l}
1 \text { if } s_{i}(t)>\operatorname{savg}_{j} \\
0 \text { otherwise }
\end{array}\right.
\end{gathered}
$$

The number of active sensors can give an idea of the concentration of pressure on the mat. When the person is lying down we would assume that more sensors would be active, than if the person was only sitting on the edge.

\section{Center of Pressure}

To improve on this solution of discerning between lying and sitting, we examined the movement of the pressure. We do this by calculating the coordinate location of the center of pressure. This solution takes into consideration both the number of active sensors and their readings. The center of pressure is calculated by finding the centroid of the image. A centroid is the geometric center of a figure in two dimensions. However since we wish to consider the value of the sensors we need to take an intensity-weighted centroid of the image. The center of pressure involves calculating the average of the sensor values in both the $\mathrm{x}$ and $\mathrm{y}$ direction. The equation for the COP in the $x$ direction is given in Equation 4. $\operatorname{PrS}(t)$ is the pressure sum from Equation $1, x_{i}$ is the column number of the sensor and $s_{i}(t)$ is the value of the sensor at sample $t$. The equation for 
the COP in the $y$ direction is given in Equation $5 . \operatorname{PrS}(t)$ is the pressure sum from Equation $1, y_{i}$ is the row number of the sensor and $s_{i}(t)$ is the value of the sensor at sample $t$. The mat is orientated in the bed so the $\mathrm{x}$ axis corresponds with horizontal movement and the $\mathrm{y}$ axis corresponds with vertical movement this is illustrated in Figure 18.

Equation 4: Center of Pressure in $\mathrm{X}$ direction

$$
\operatorname{COPx}(t)=\frac{1}{\operatorname{PrS}(t)} \sum_{i=1}^{72} x_{i} s_{i}(t)
$$

Equation 5: Center of Pressure in Y direction

$$
\operatorname{COPy}(t)=\frac{1}{\operatorname{PrS}(t)} \sum_{i=1}^{72} y_{i} s_{i}(t)
$$




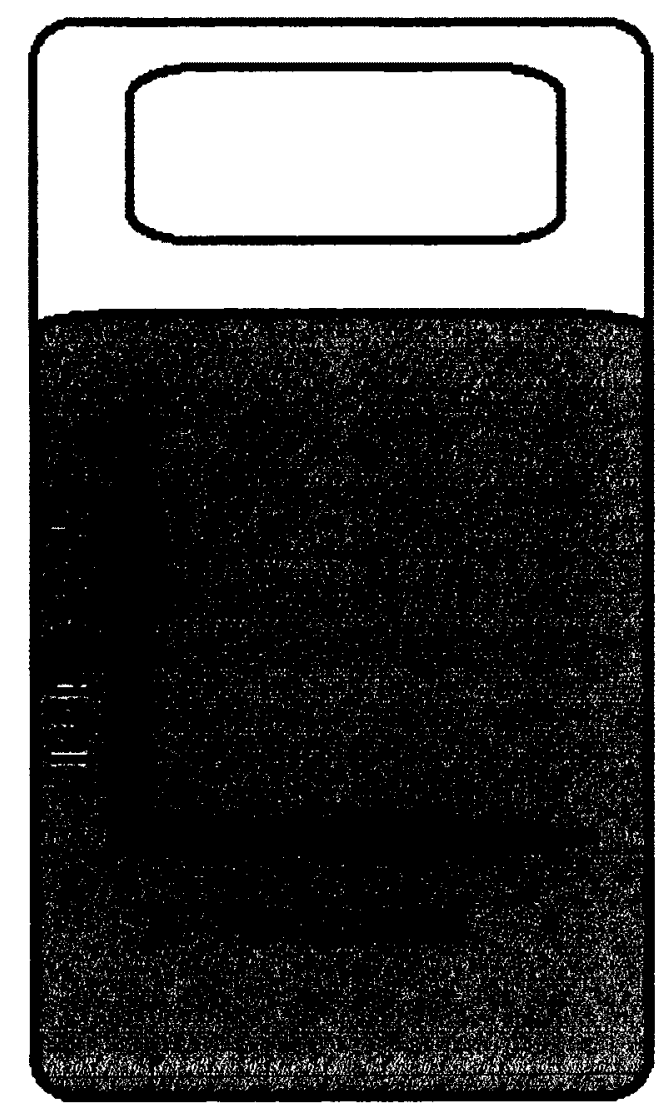

Figure 18: Directions of center of pressure

\section{Example: Center of Pressure}

Calculating the center of pressure is best explained using a visual example. First we will create a simple image with the dimensions $9 \times 8$; this is the same dimensions of an image that would be formed using the bed mat. We will place a rectangular image structure towards the upper right hand side. The values of the pixels or simulated sensor readings will be binary to simply the calculation. The image is shown in Figure 19. 


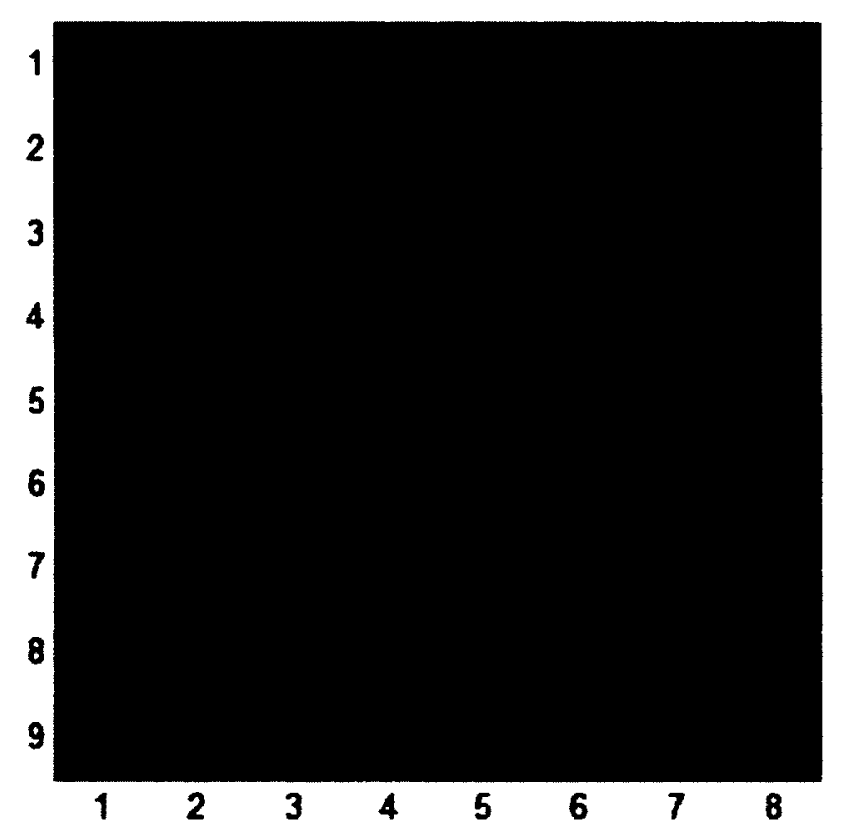

Figure 19: Basic image used for COP calculation

The image can be represented by the matrix shown in Table 3. The first step in the calculation is to create two new matrices with each value or pixel set to its $\mathrm{x}$ or $\mathrm{y}$ coordinate respectively. Table 4 shows the matrix created for solving the COP in the $\mathrm{x}$ direction; Table 5 shows the matrix created that will be used for the $y$ direction.

Table 3: Matrix representation of basic image for COP calculation

\begin{tabular}{|llllllll|}
\hline 0 & 0 & 0 & 0 & 0 & 0 & 0 & 0 \\
0 & 0 & 0 & 0 & 1 & 1 & 1 & 0 \\
0 & 0 & 0 & 0 & 1 & 1 & 1 & 0 \\
0 & 0 & 0 & 0 & 1 & 1 & 1 & 0 \\
0 & 0 & 0 & 0 & 1 & 1 & 1 & 0 \\
0 & 0 & 0 & 0 & 1 & 1 & 1 & 0 \\
0 & 0 & 0 & 0 & 0 & 0 & 0 & 0 \\
0 & 0 & 0 & 0 & 0 & 0 & 0 & 0 \\
0 & 0 & 0 & 0 & 0 & 0 & 0 & 0 \\
\hline
\end{tabular}


Table 4: Matrix for solving COP in $\mathrm{x}$ direction

\begin{tabular}{|llllllll|}
\hline 1 & 2 & 3 & 4 & 5 & 6 & 7 & 8 \\
1 & 2 & 3 & 4 & 5 & 6 & 7 & 8 \\
1 & 2 & 3 & 4 & 5 & 6 & 7 & 8 \\
1 & 2 & 3 & 4 & 5 & 6 & 7 & 8 \\
1 & 2 & 3 & 4 & 5 & 6 & 7 & 8 \\
1 & 2 & 3 & 4 & 5 & 6 & 7 & 8 \\
1 & 2 & 3 & 4 & 5 & 6 & 7 & 8 \\
1 & 2 & 3 & 4 & 5 & 6 & 7 & 8 \\
1 & 2 & 3 & 4 & 5 & 6 & 7 & 8 \\
\hline
\end{tabular}

Table 5: Matrix for solving COP in y direction

\begin{tabular}{|llllllll|}
\hline 1 & 1 & 1 & 1 & 1 & 1 & 1 & 1 \\
2 & 2 & 2 & 2 & 2 & 2 & 2 & 2 \\
3 & 3 & 3 & 3 & 3 & 3 & 3 & 3 \\
4 & 4 & 4 & 4 & 4 & 4 & 4 & 4 \\
5 & 5 & 5 & 5 & 5 & 5 & 5 & 5 \\
6 & 6 & 6 & 6 & 6 & 6 & 6 & 6 \\
7 & 7 & 7 & 7 & 7 & 7 & 7 & 7 \\
8 & 8 & 8 & 8 & 8 & 8 & 8 & 8 \\
9 & 9 & 9 & 9 & 9 & 9 & 9 & 9 \\
\hline
\end{tabular}

The second step is to use the sensor values or pixel values to modify the $\mathrm{x}$ and $\mathrm{y}$ COP solving matrices. Quite simply we multiply the original image pixel values by the values in the solving matrices. The result is shown in Table 6 and Table 7.

Table 6: Modified solving matrix for COP $\mathrm{x}$ direction

\begin{tabular}{|llllllll|}
\hline 0 & 0 & 0 & 0 & 0 & 0 & 0 & 0 \\
0 & 0 & 0 & 0 & 5 & 6 & 7 & 0 \\
0 & 0 & 0 & 0 & 5 & 6 & 7 & 0 \\
0 & 0 & 0 & 0 & 5 & 6 & 7 & 0 \\
0 & 0 & 0 & 0 & 5 & 6 & 7 & 0 \\
0 & 0 & 0 & 0 & 5 & 6 & 7 & 0 \\
0 & 0 & 0 & 0 & 0 & 0 & 0 & 0 \\
0 & 0 & 0 & 0 & 0 & 0 & 0 & 0 \\
0 & 0 & 0 & 0 & 0 & 0 & 0 & 0 \\
\hline
\end{tabular}


Table 7: Modified solving matrix for COP y direction

\begin{tabular}{|llllllll|}
\hline 0 & 0 & 0 & 0 & 0 & 0 & 0 & 0 \\
0 & 0 & 0 & 0 & 2 & 2 & 2 & 0 \\
0 & 0 & 0 & 0 & 3 & 3 & 3 & 0 \\
0 & 0 & 0 & 0 & 4 & 4 & 4 & 0 \\
0 & 0 & 0 & 0 & 5 & 5 & 5 & 0 \\
0 & 0 & 0 & 0 & 6 & 6 & 6 & 0 \\
0 & 0 & 0 & 0 & 0 & 0 & 0 & 0 \\
0 & 0 & 0 & 0 & 0 & 0 & 0 & 0 \\
0 & 0 & 0 & 0 & 0 & 0 & 0 & 0 \\
\hline
\end{tabular}

The third step is to sum all the values in each of the modified matrices and divide this by the sum of the values in the original image (PrS shown in Equation 1). The result gives us the coordinate location of the center of pressure in the $\mathrm{x}$ and $\mathrm{y}$ direction. It is shown below.

COP-x Direction:

Sum of values in table $5=90 \quad \operatorname{PrS}=15$

$$
\operatorname{COP}-\mathrm{x}=(90 / \operatorname{PrS})=(90 / 15)=6
$$

COP-x Direction:

Sum of values in table $6=60 \quad \operatorname{PrS}=15$

$$
\operatorname{COP}-\mathrm{y}=(60 / \operatorname{PrS})=(60 / 15)=4
$$

The center of pressure is at $(6,4)$. For the purpose of this example, the COP has been overlaid on the original image and is shown in red. This is shown in Figure 20. 


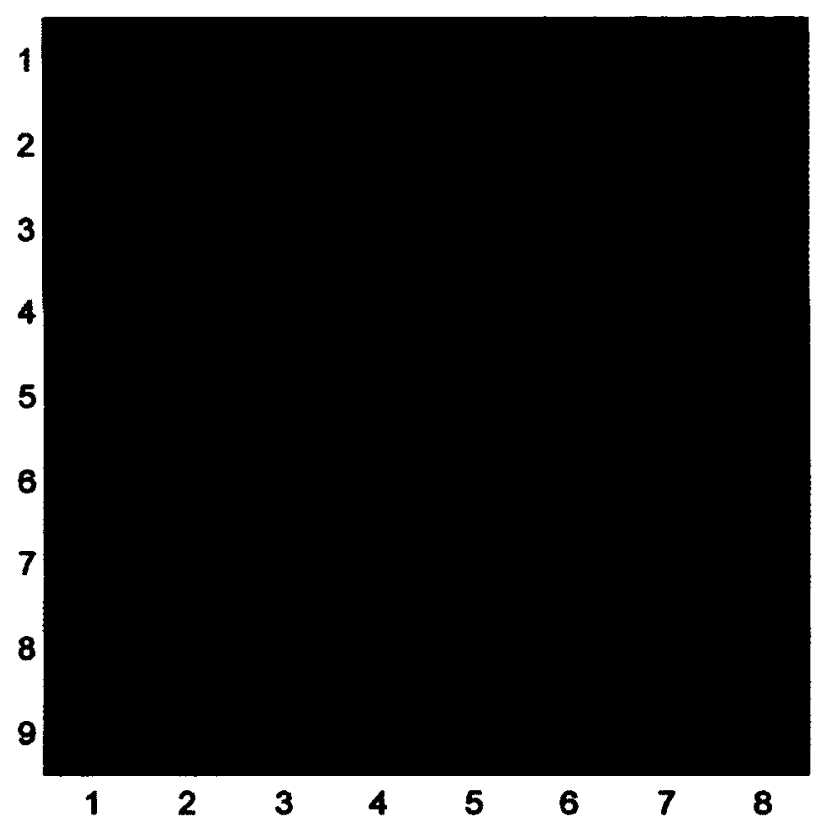

Figure 20: Basic image for COP calculation solved. COP in red.

\section{Observing features over time}

Three features have been defined, the pressure sum $(\operatorname{PrS})$, the pressure concentration determined by the number of active sensors (NumActive), and the coordinates of the center of pressure (COP-x, COP-y). The pressure recordings span over months however we can generate plots over any amount of time, to determine the best feature for extracting clinical information we plotted each of them over a 24 hour period.

The 24 hour period starts at 12:00 (noon) day 1 and ends 12:00 (noon) on day 2. This allows us to plot the entire night time, presuming the participant sleeps at night this is where the important data resides. Figure 21 is a 24 hour plot of the pressure sum with the $x$ axis in the actual time of day and a second plot in seconds; the two other plots are the coordinate locations for the center 
of pressure, the $\mathrm{x}$ direction and the $\mathrm{y}$ direction for one participant. By briefly observing the plots we see there are two distinct portions, the first when the features have a fixed value, and where there is constant fluctuation. It is intuitive that when the feature values are fixed there is no activity seen by the pressure mat. From the 24 hour plots we want to extract clinically relevant information.
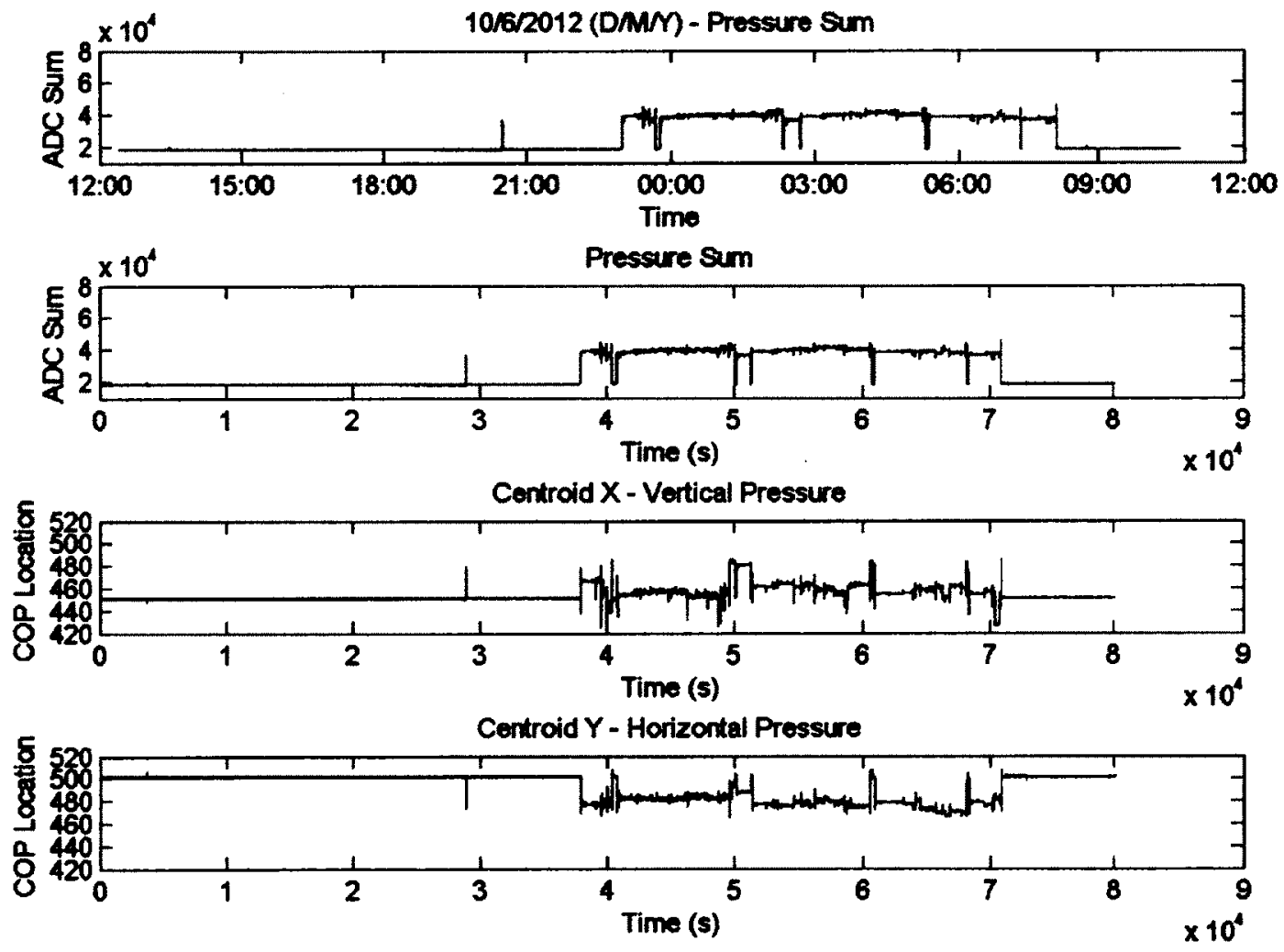

Figure 21: Example of features for 24 hour recording from P382 


\subsubsection{Clinical feature extraction}

There are numerous clinically relevant features we can extract from the pressure mat data. Some simple features are the amount of total time spent in bed, number of bed exits, hours of continuous bed occupancy, time of exit. Clinical features related to bed exit and entries require more complex processing. All of these features are important for patient monitoring; we will now discuss how they are extracted.

\section{Bed occupancy features}

The first set of features are related to bed occupancy, they are simple to calculate, but are required as a pre-processor for more complex features. Bed occupancy is determined by using either the pressure sum (PrS) or the number of active sensors (NumActive). A threshold for either feature is specified and the data is scanned through, when the PrS meets the threshold the sample number is logged. We continue scanning through the data, when the feature value drops below the threshold, that sample number is logged. We then take the time difference between the samples to determine duration of the bed occupancy. The entire data range (generally 24 hours at a time) is scanned through. Every time bed occupancy is detected we log the timestamp under clinical feature "bed entry time", when lack of occupancy is detected we log the timestamp under "bed exit time", these two clinical features are exported into a CSV file. The duration of the bed occupancy is calculated and exported. The total bed occupancy time is the sum of all the bed occupancy during the data range, this feature is exported. The number of bed exits is the sum of the "bed exit time" feature and this is exported. These features give clinicians with information 
to help monitor the patient's sleep habits and routine. It can help find abnormalities in the sleep pattern that the patient may not realize themselves.

The bed occupancy features are summarized in the example shown in Table 8 . There were 3 entries and exits detected. The durations are expressed in seconds.

Table 8: Example showing bed occupancy features

\begin{tabular}{|l|l|l|l|}
\hline Bed entry time & 13.26 .37 .312 & 13.36 .45 .359 & 13.51 .07 .171 \\
\hline Bed exit time & 13.31 .41 .750 & 13.37 .10 .875 & 14.13 .23 .734 \\
\hline Duration (s) & 304.4 & 25.55 & 1336.45 \\
\hline Total bed occupancy duration (s) & 1666.4 & \\
\hline Total number of bed exits & 3 & \\
\hline
\end{tabular}

\section{Bed exit times}

The more complex clinical features are the bed exit and entry measures. These include the lie-tosit and sit-to-stand times. One of the objectives of this research was to automate the process of measuring these clinical features. Generally the bed exit and entry times are less than 5 minutes, and with hours of pressure data, we need to quickly scan through it to the data range required to perform these measures. To determine the bed exit timings we work backwards from the exit time, determining bed occupancy was simple using a threshold based pressure sum. We zoom in on the data range that allows us to observe all the exit features. Using the same 24 hour data set used to create Figure 21, we have manually zoomed into the last bed exit in the recording. This was at approximately 8 am. The zoomed plots are shown in Figure 22. 

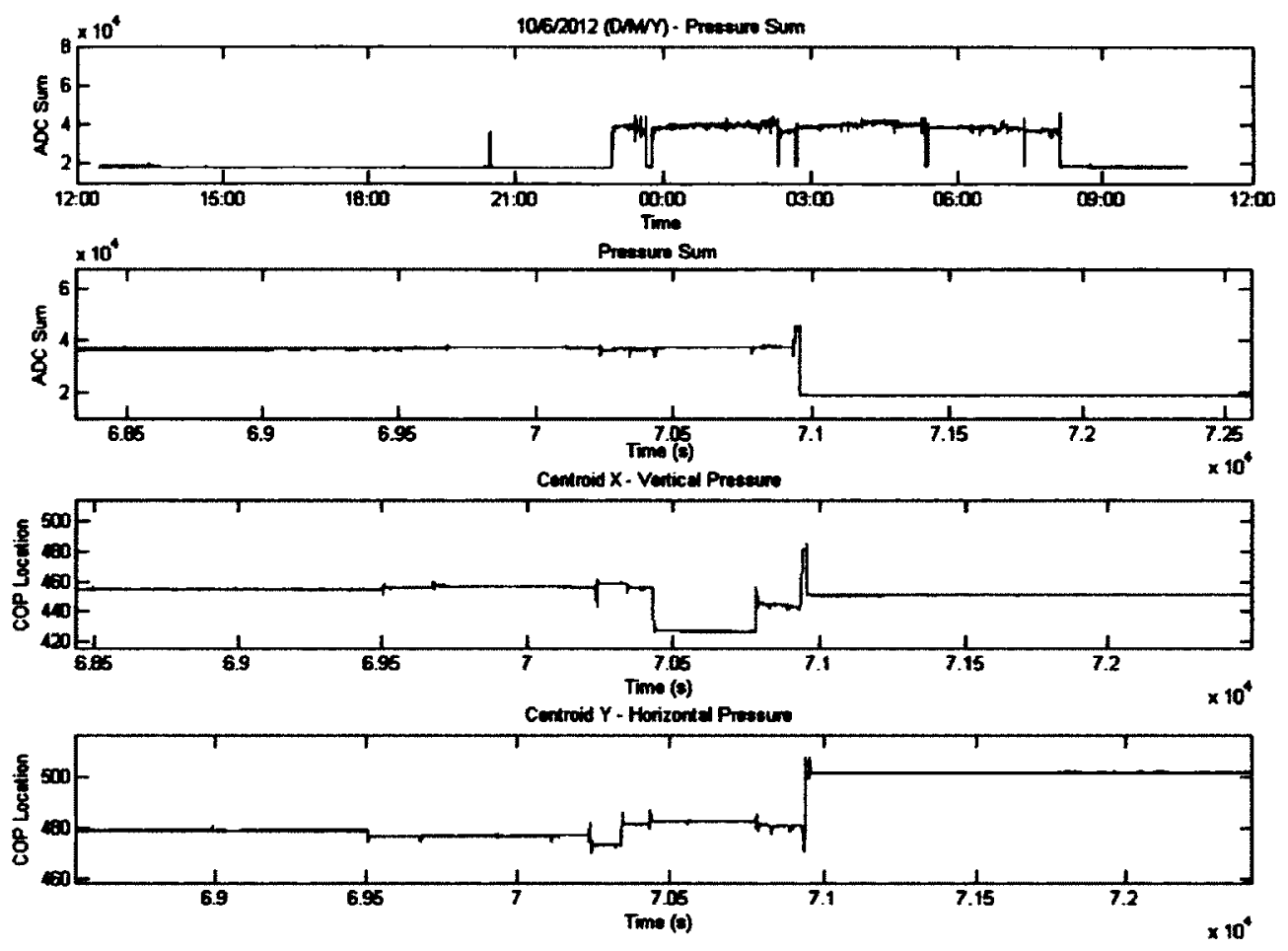

Figure 22: Zoomed in region of the bed exit
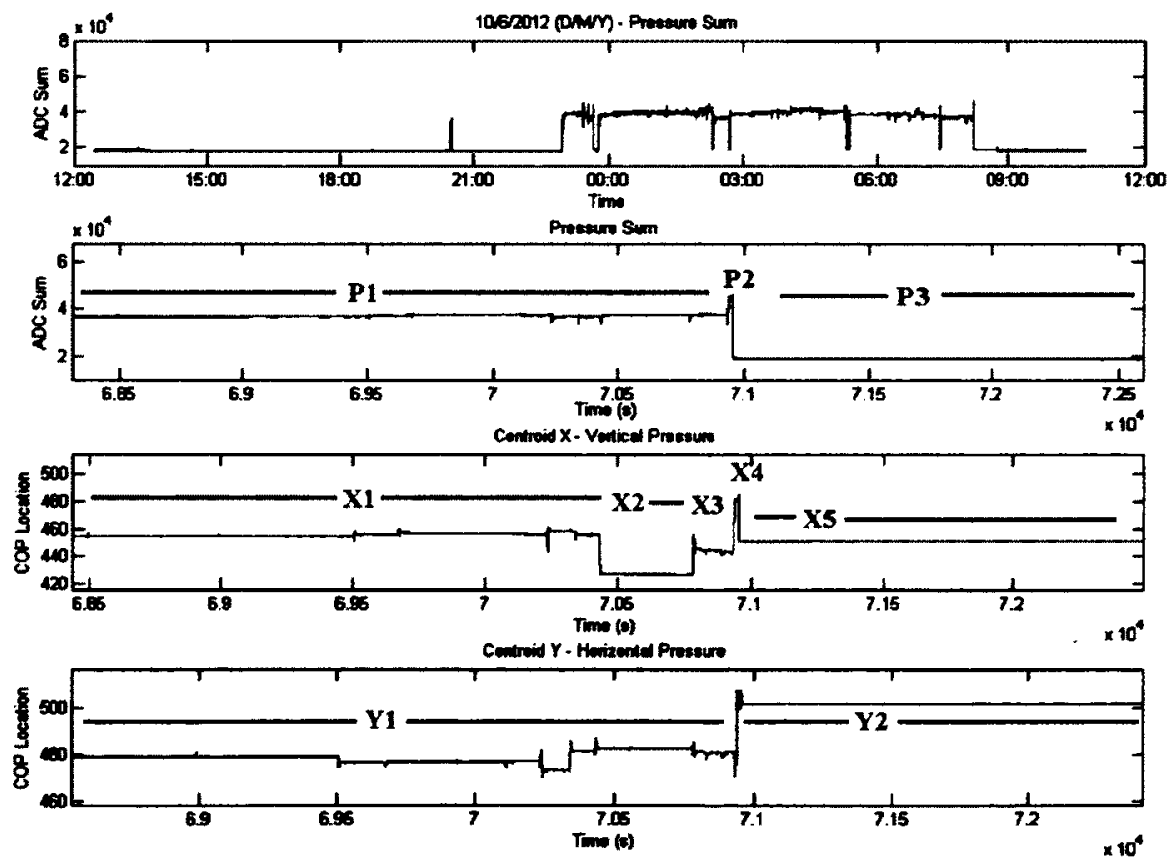

Figure 23: Zoomed in region of the bed exitwith annotations 


\section{Example: Manual bed exit timing extraction}

Figure 23 is identical to Figure 22 however it has been annotated with the various states that will be described in this example. By visually examining the plots we can see the patient has exited that bed just before $7.1 \times 10^{4}$ seconds. The pressure sum plot shows a distinct change in state from a steady $3.8 \times 10^{4}$ (State P1) to a brief increase to $4.2 \times 10^{4}$ (State P2) to a drop to $2 \times 10^{4}$ (State P3) where it remains constant. Using the COP in the $\mathrm{x}$ direction we see more changes in state, the signal is fairly steady around 455 (State X1), then it drops abruptly to 425 (State X2). It then increases to 440 (State X3), then are large brief increase to 480 (State X4), before dropping to 450 (State X5) and remaining flat. Using the COP in the $y$ direction the values fluctuate around 480 (State Y1), then jump to 500 (State Y2) just before the exit.

The components of the exit time we are trying to extract are the lie-to-sit and sit-to-stand times. Therefore we need to be able to determine the patient's state from the feature values. The best feature to use for this example is the COP in the $\mathrm{x}$ direction. A zoomed in plot of only COP-x during the bed exit transfer is shown in Figure 24. 


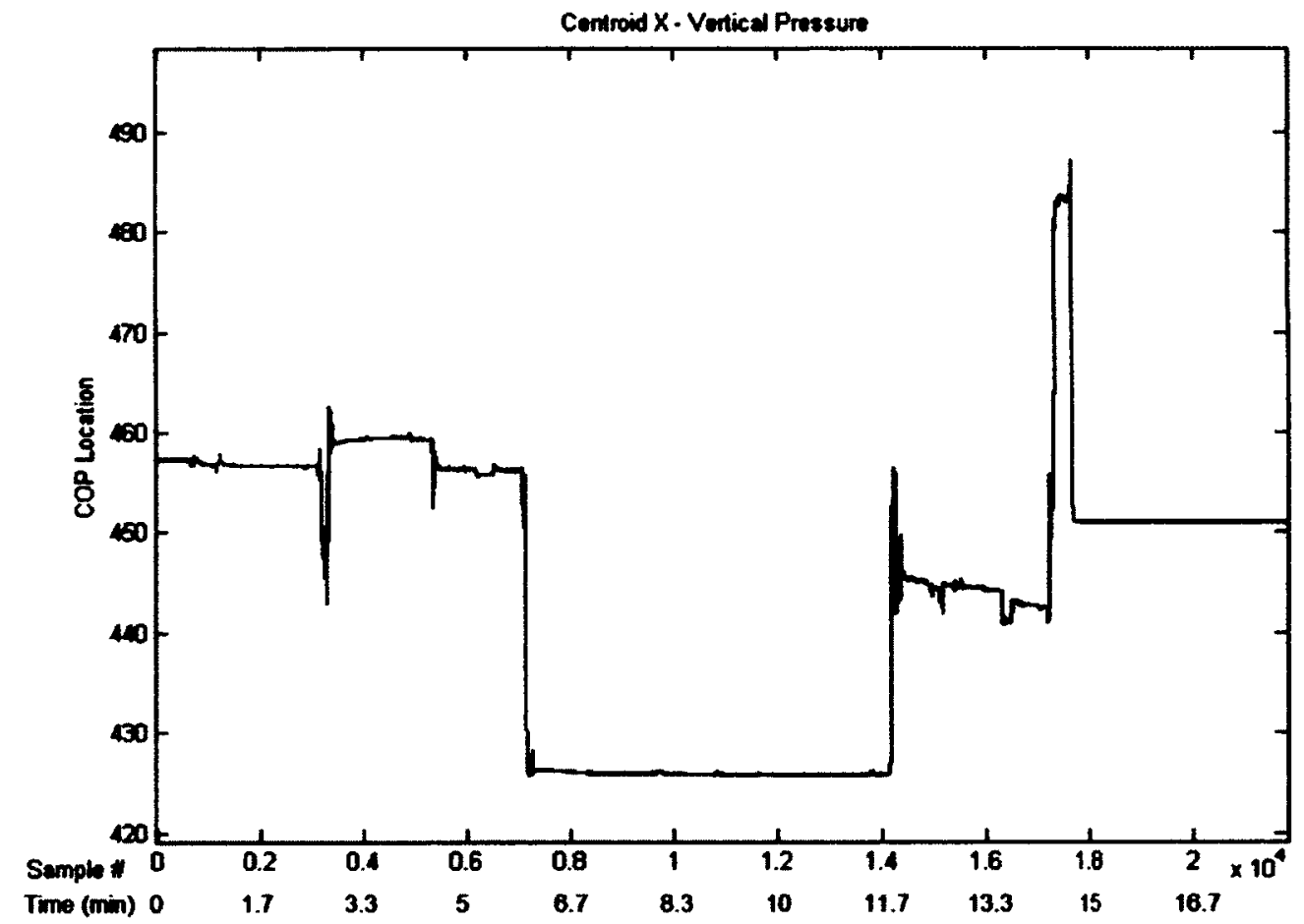

Figure 24: Bed exit transfer using COP-x feature

Various points of interest were labelled. As shown in Figure 25. Each of the points will be examined using pressure mapped images to determine the patient's position in the bed. This will allow us to determine appropriate start and end points for lie-to-sit and sit-to-stand transfers. 


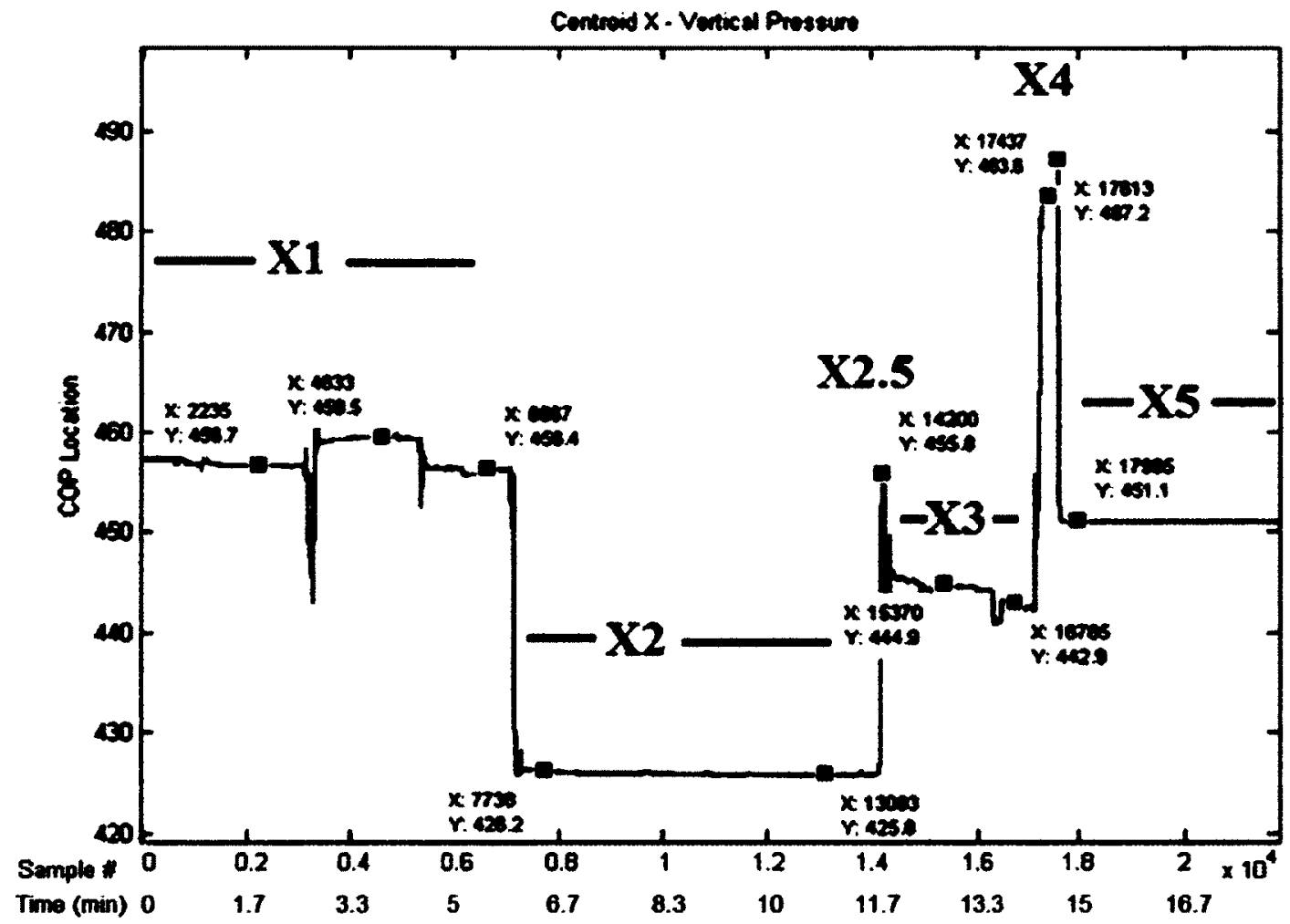

Figure 25: Bed exit transfer using COP-x feature. Annotated with points of interest

When the value was initially 456 , we called this state $\mathrm{X} 1$ the patient is lying down, they then change positions this is reflected in the center of pressure moving lower on the mat to a value of 426, we called this state $X 2$. This holds steady for over 5 minutes. There is then another change in position which causes the center of pressure to move upwards, it peaks at 455 , we will call this state $\mathrm{X} 2.5$, and then evens out around $\mathbf{4 4 5}$ for several minutes, this is state $\mathrm{X} 3$. There is another change in position, the center of pressure moves upwards, this is because the patient has moved into the sitting position, and this is called state $\mathrm{X} 4$. There is a short period of time in the sitting position before they exit the bed and the center of pressure drops to 451 , this is state X5. 
Pressure mapped images were created from each of the labelled points in Figure 25 . The images were generated using both non-filtering and smooth-filtering methods, both will be shown. State $\mathrm{X} 1$ contained 3 points these are shown in Figure 26 . In state $\mathrm{X} 1$ the patient is in the lying position, this is determined by manual visual analysis.

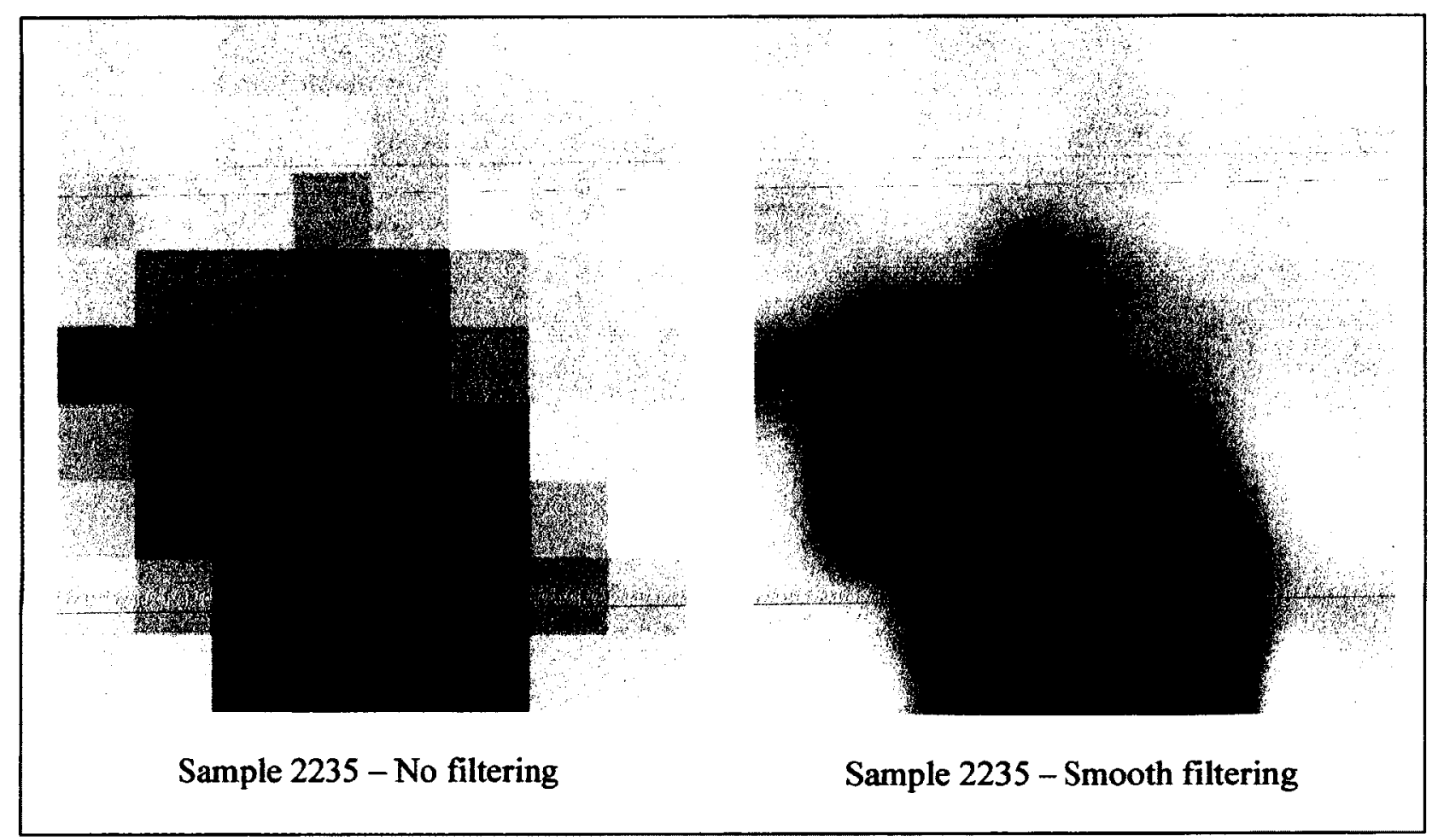




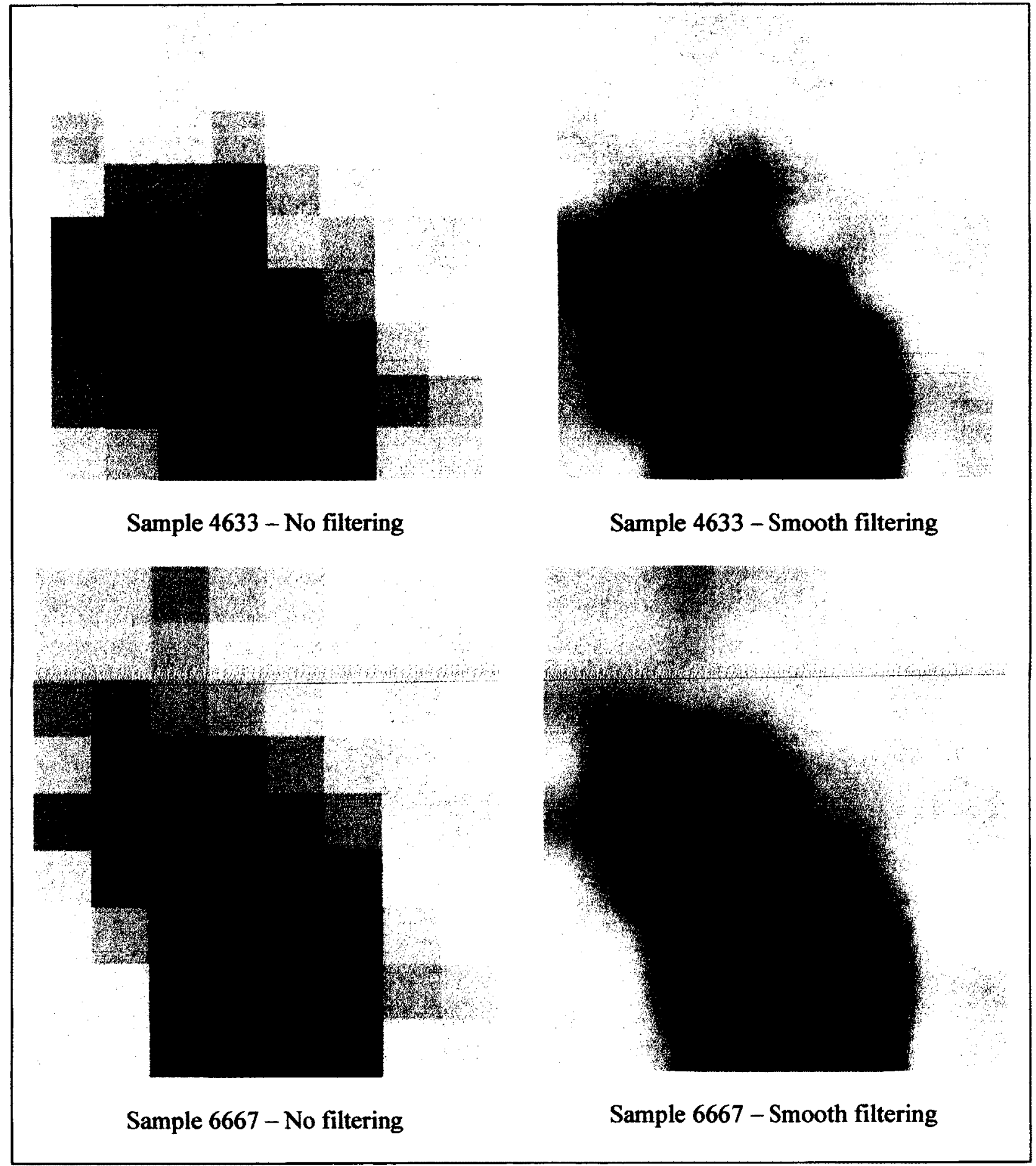

Figure 26: Pressure mapped images of patient lying in state $\mathrm{X} 1$ 
State X2 contained 2 points these are shown in Figure 27 . In state $X 2$ the patient is in the lying position, however they have rolled over to the right side of the bed from the center of the bed in state $\mathrm{X} 1$, this was determined by manual visual analysis. 


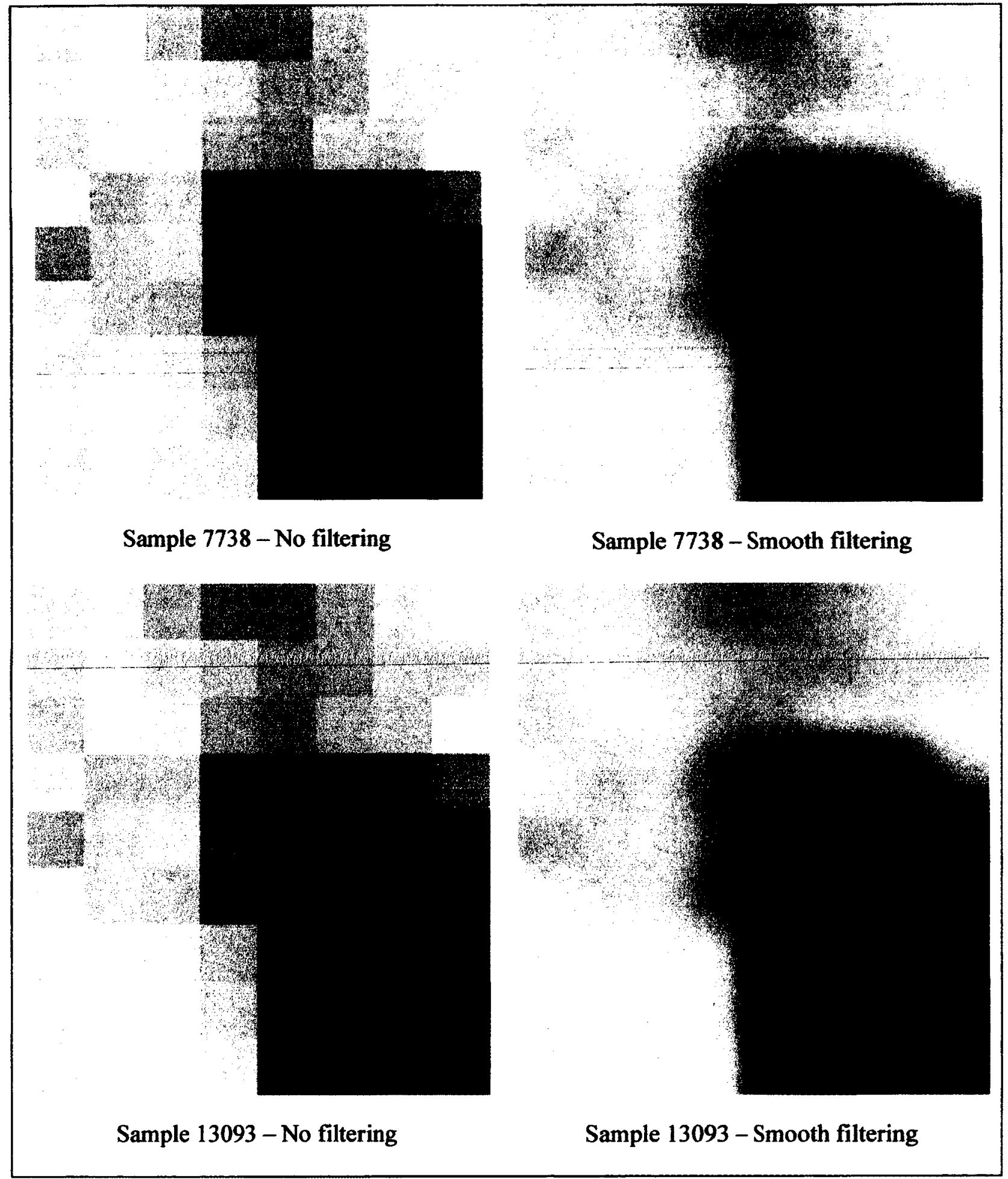

Figure 27: Pressure mapped images of patient lying in state $\mathrm{X} 2$ 
State X2.5 contained 1 point, pressure mapped images were created and are shown in Figure 28 . In state $\mathrm{X} 2.5$ the patient is in the lying position, however they have rolled over from the right side of the bed back to the center of the bed, the same position we saw in state $X 1$, this was determined by manual visual analysis.

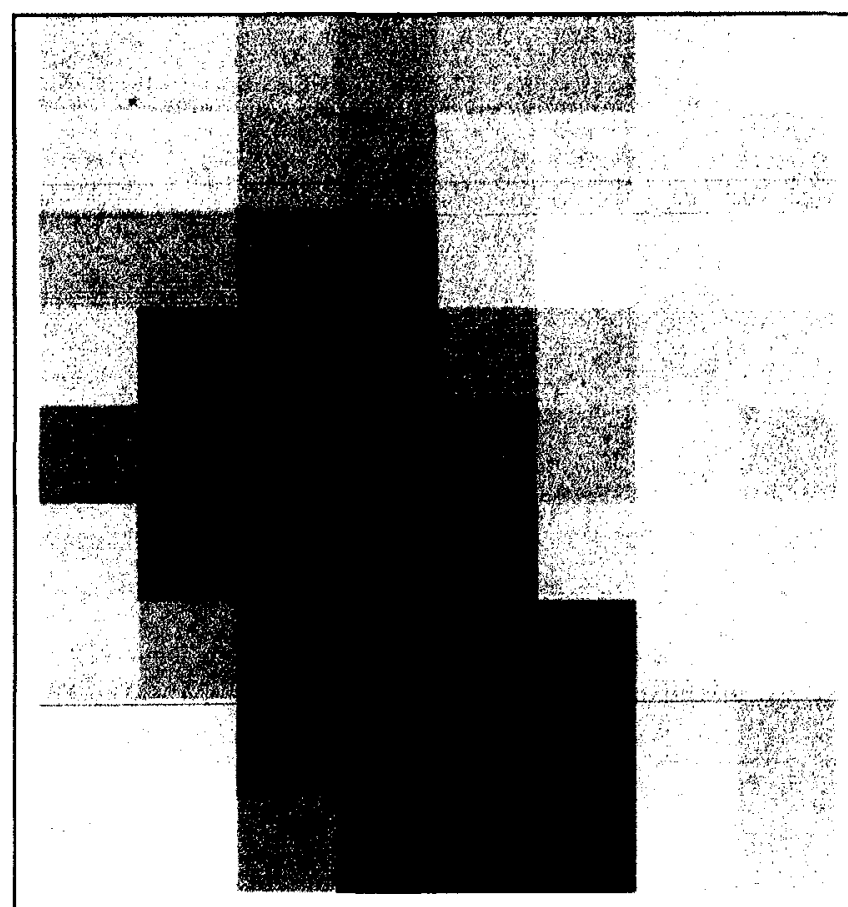

Sample 14200 - No filtering

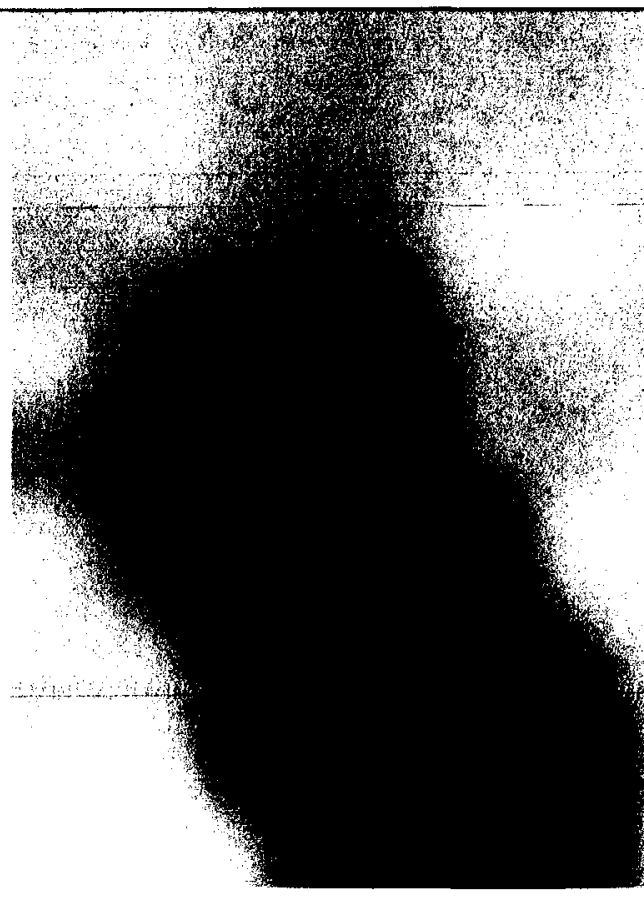

Sample 14200 - Smooth filtering

Figure 28: Pressure mapped images of patient lying in state X2.5

State X3 contained 2 points these are shown in Figure 29. In state X3 the patient is in the lying position, however they have shifted their weight slightly as compared to state $\mathrm{X} 2.5$, this is the final lying position before they begin the LtS transfer, and this was determined by manual visual analysis. 


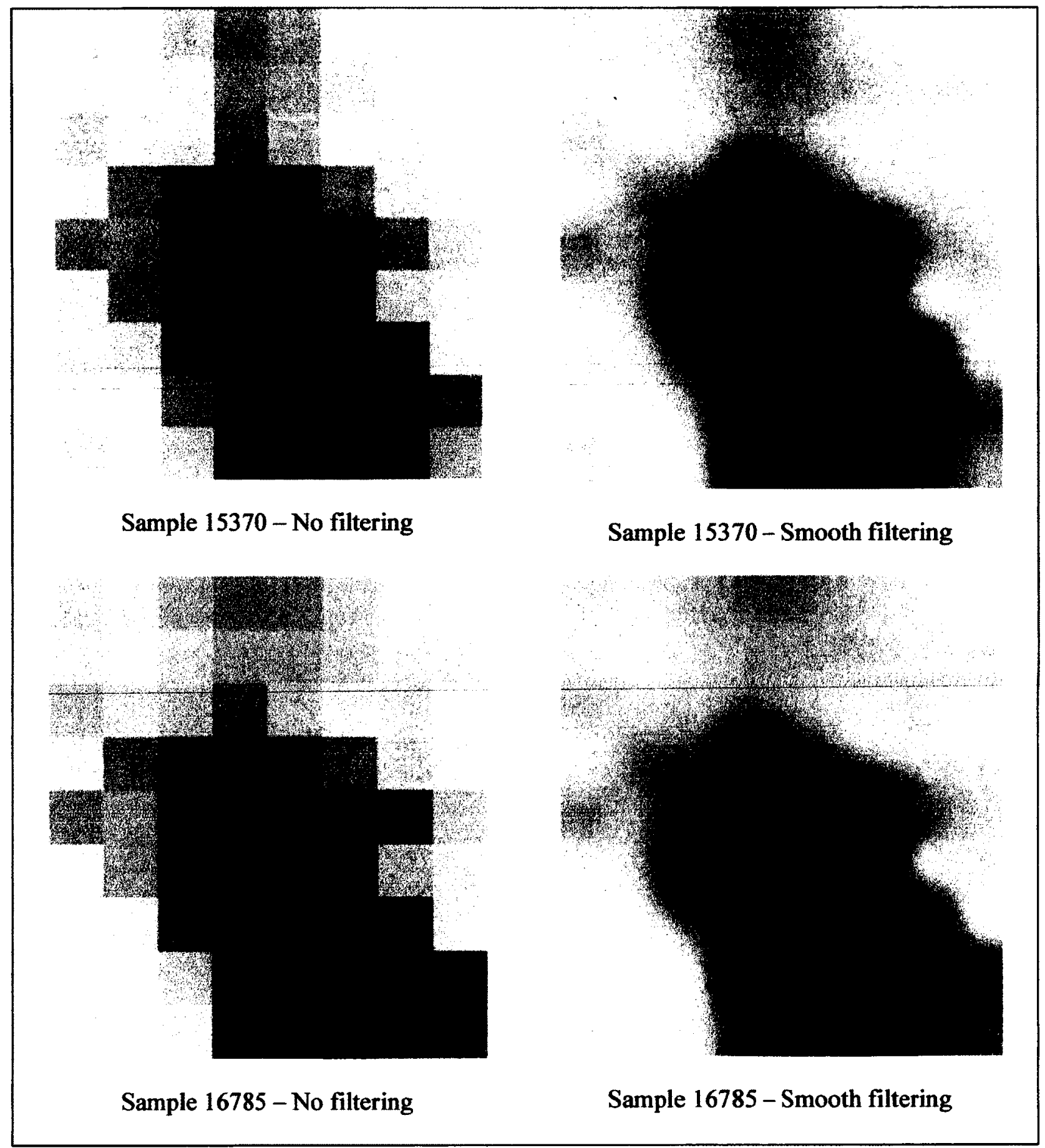

Figure 29: Pressure mapped images of patient lying in state X3

State $\mathrm{X} 4$ contained 2 points these are shown in Figure 30. In state $\mathrm{X} 4$ the patient is in the sitting position, they have transferred from the lying position in state X3. In the second sample of state 
$\mathrm{X} 4$ the pressure is moving closer to the left edge this is because this is the start of the StS transfer, and this was determined by manual visual analysis.

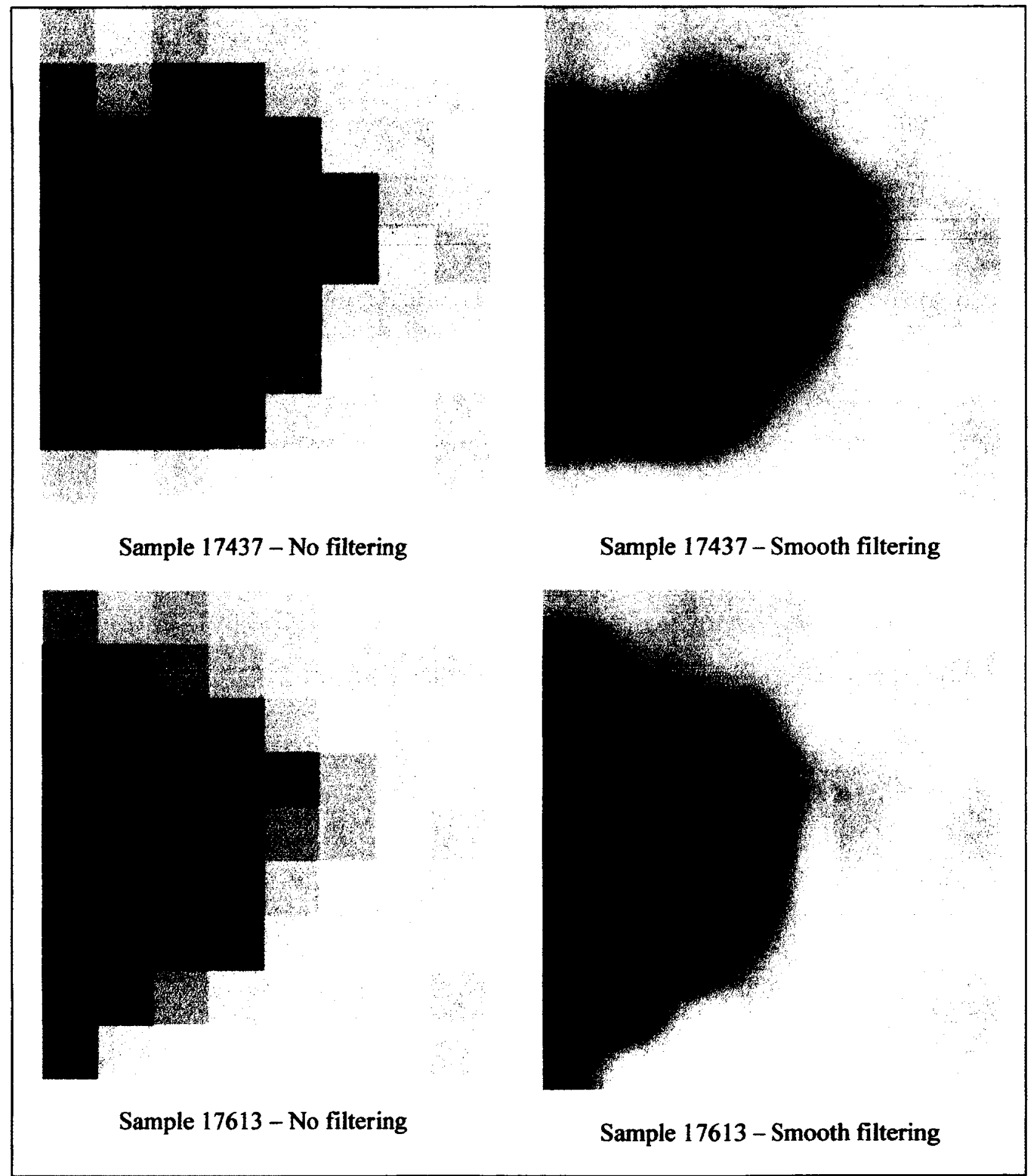

Figure 30: Pressure mapped images of patient in sitting position in state $\mathrm{X} 4$ 
State X5 contained 1 point and is shown in Figure 31. In state X5 the patient has successfully completed the StS transfer and is out of the bed. The pressure mapped image is inherently blank as there is no longer and weight on its sensor field.

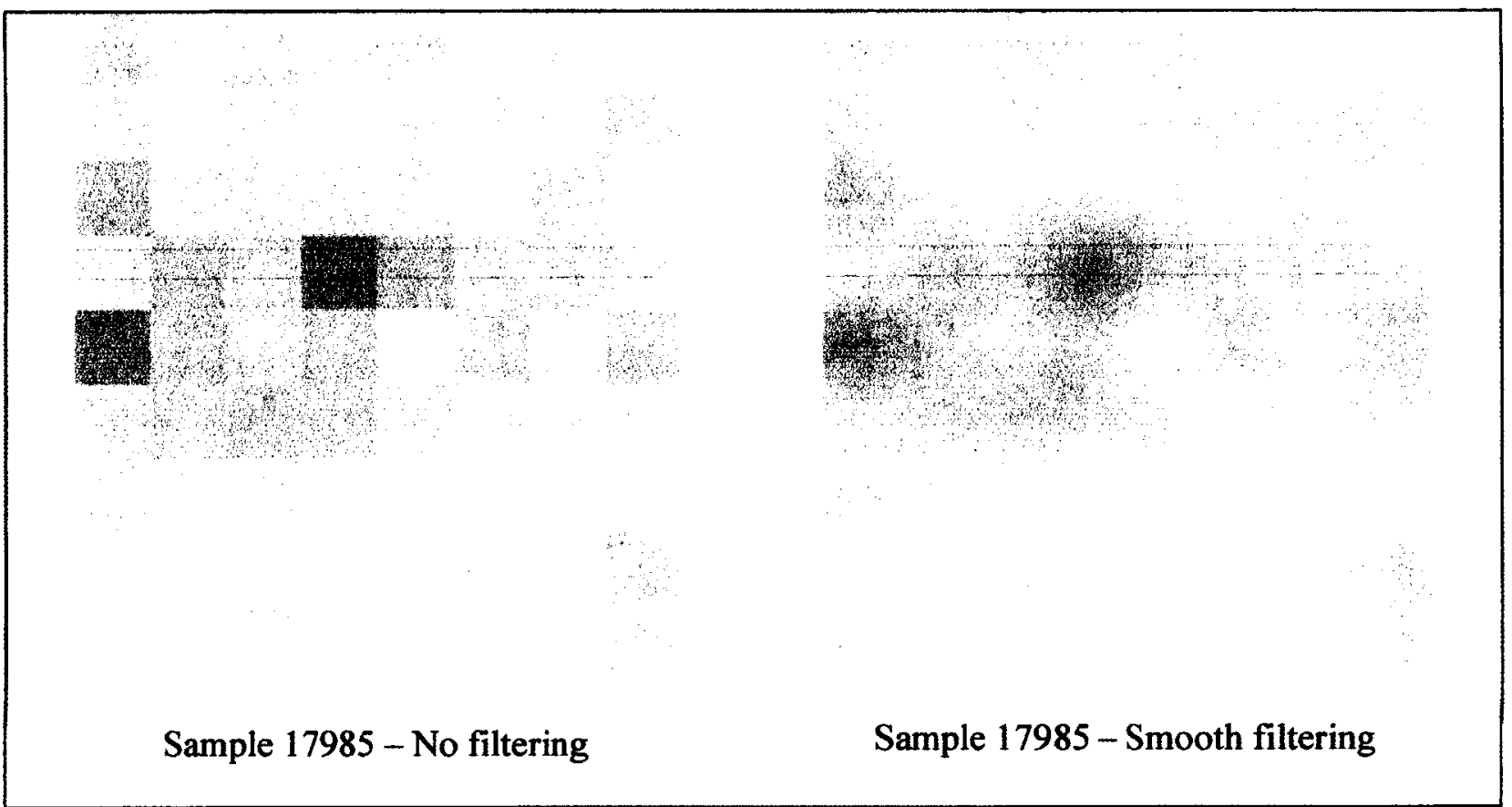

Figure 31: Pressure mapped images of patient out of bed, state X5

Using the pressure mapped images in combination with the extracted features of PrS, COP-x and COP-y we are able to determine what position the patient is in. Using this information we are able to extract the bed exit timings, lie-to-sit, sit duration, and sit-to-stand times. By manually processing a couple exits from a single patient a pattern is usually found. We found the patient generally exits in the same manor, which is they move from a lying position to a sitting position to an exit. The extracted features related to the center of pressure for each position is examined and we can find a consistent range. Once the normal ranges for the COP-x or COP-y values are determined we can use software automation to calculate the bed exit timings, without the need of manual visual analysis of pressure mapped images. 


\subsubsection{Exporting data, plots, images}

The software is designed to give every option available for data output. Single recording files to multiple files can be loaded. This gives the operator the ability to process a batch of files quickly with requiring any input after the setup procedure. Once the data is loaded and export directory is specified. The following exported information can be selected:

(1) Generate plots for the following features:

a. PrS, COP-x, COP-y

b. Use sample number, seconds, or actual time of day for $\mathrm{x}$-axis

c. Generate plots for each file ( 1 hour) or over several hours, the start and end time must be specified, usually this is 12 noon -12 noon the following day.

(2) Export features to a CSV file, this could include:

a. PrS, COP-x, COP-y

(3) Detect bed occupancy and export:

a. No activity, continuous activity, and bed entry and exit timestamps

(4) Process the bed exits and extract clinical information

a. Export LtS, Sit time, StS times to CSV

(5) Create pressure mapped images and save in PNG format

(6) Create pressure playback video and save in AVI format

All of the options are available from the main GUI, it should be noted that option (3) must be selected in order to perform option (4). 


\subsubsection{Implementation of automation}

One of the objectives of this thesis was to develop a robust processing algorithm that would be able to handle the long term pressure mat recordings. Essentially we needed an automated system for extracting the clinical information. A flowchart was created with 5 main steps in the processing algorithm, 1) loading data, 2) preprocessing, 3) extracting signal features, 4) extracting clinical features, 5) exporting processed data. The flowchart is shown in Figure 32.

The first stage of the algorithm is loading the data; a flowchart showing the individual steps in this stage was created and shown in Figure 33. As stated previously, single or multiple recording files can be loaded. The data is then sorted into chronological order. The recording files are checked for any discrepancies in start and end times. If a recording file is shorter that the specified recording time of 1 hour, then we know a failure has occurred, and the operator is informed. After checking the files for integrity they can be opened and the values can be read. We now move on to stage 2 , the preprocessing. 


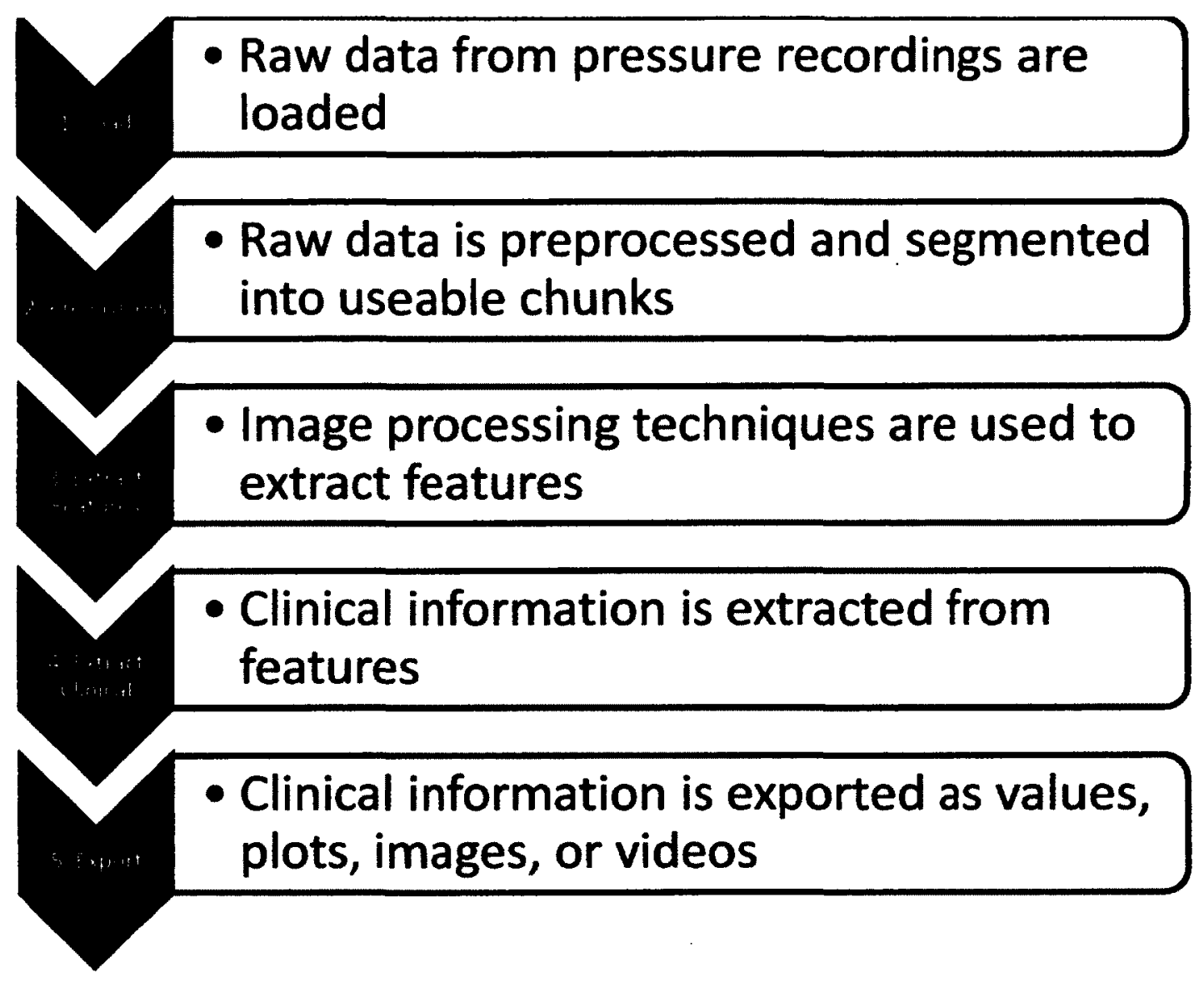

Figure 32: Flowchart of automated processing algorithm for bed mat sensor 


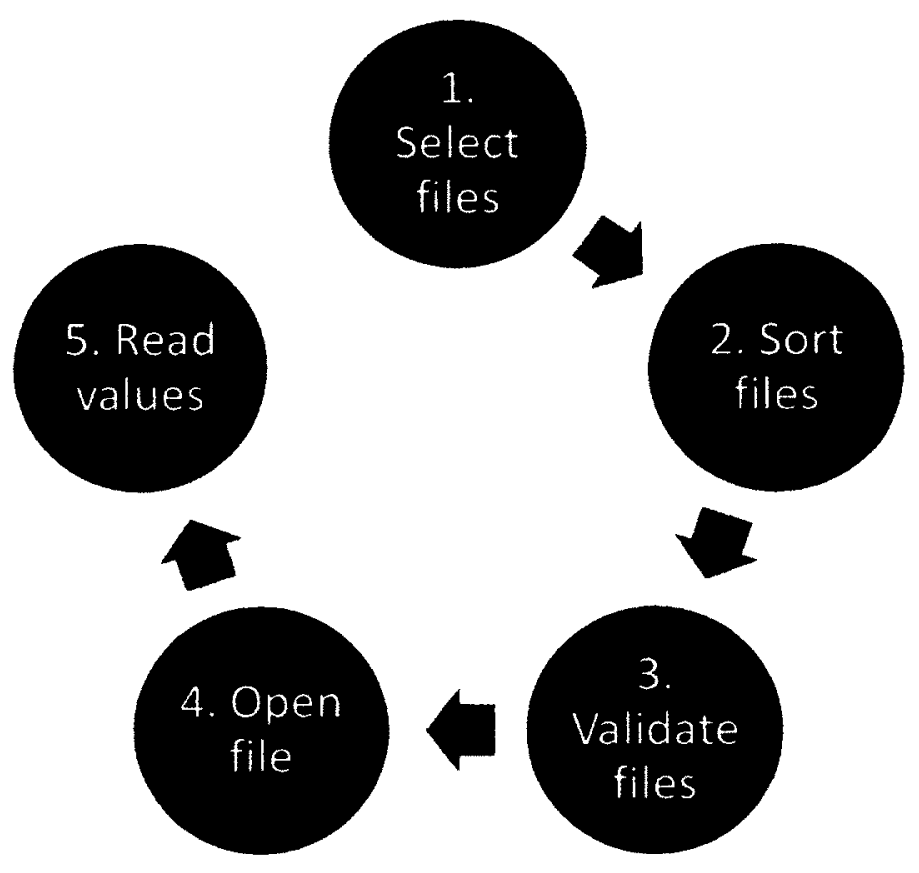

Figure 33: Flowchart for Step (1) Loading data

Stage 2 deals with the preprocessing required before extracting the clinical information, the major steps involved are summarized in the flowchart shown in Figure 34. To automate the extraction of clinical features from the pressure data we started with quickly scanning through the data and classifying each recording as having:

1) No activity

2) Continuous occupancy (sleep)

3) Entry or Exit has occurred

The classifier is based on a threshold value set on the pressure sum (PrS) feature. When the patient data is less than the threshold for the entire recording it is classified as (1) no activity. When the patient data is greater than the threshold for the entire recording it is classified as (2) continuous occupancy. If the recording begins with no activity, then the threshold is met, the 
timestamp this occurs is logged as an entry, class (3). If the recording has activity then the PrS drops below the threshold this event is logged as an exit under class (3).

Using this simple scan and classification we are able to extract the bed occupancy features from section 4.2.2:

1) Export the time at which a bed entry occurs

2) Export the time at which a bed exit occurs

3) Calculate the number of entries

4) Calculate the number of exits

5) Calculate the each bed occupancy time

6) Calculate the total bed occupancy time over 24 hours

The software creates a CSV file with the first column the name of the recording file processed. Then it creates a column for each entry and exit that has occurred with the time. An example of this is shown in Table 9.

Table 9: Bed occupancy detection results

\begin{tabular}{|l|l|l|l|}
\hline$\#$ & Filename & Entry & Exit \\
\hline 1 & 'data_sn4353_2012_01_21_23_56_38' & 0 & 0 \\
2 & 'data_sn4353_2012_01_22_00_56_39' & 0 & 0 \\
3 & 'data_sn4353_2012_01_22_01_56_40' & 0 \\
4 & 'data_sn4353_2012_01_22_02_56_41' & & 0 \\
5 & 'data_sn4353_2012_01_22_03_56_42' & 0 \\
6 & 'data_sn4353_2012_01_22_04_56_43' & \\
7 & 'data_sn4353_2012_01_22_05_56_44' & 0 \\
8 & 'data_sn4353_2012_01_22_06_56_45' & 0 \\
9 & 'data_sn4353_2012_01_22_07_56_46' & 0 \\
10 & 'data_sn4353_2012_01_22_08_56_47' & 0 \\
11 & 'data_sn4353_2012_01_22_09566_48' & 0 & 0 \\
12 & 'data_sn4353_2012_01_22_10_56 49' & 0 & 0 \\
\hline
\end{tabular}


We see that the first two files have 0 s for the entry and exit columns, this is because no activity was detected. The third file shows a value of 11353 in the entry column and a 0 in the exit column, this means that an entry was detected at sample time 11353 and remained continuous activity throughout the recording. Files $4-9$ have a 1 in the entry column and 0 in the exit column, this means that at the beginning of the recording there was activity and it was continuous. The $10^{\text {th }}$ file begins with activity, signified by the 1 in the entry column, however in the exit column the value is $\mathbf{4 2 3 4 5}$. This means there was a bed exit at sample time 42345 .

In summary, this example shows there was a bed entry at sample time 11353 in file 3 , and then there was continuous bed occupancy until sample time 42345 in file 10 . There was 1 bed entry and 1 bed exit over this 12 hour of data set. The total bed occupancy time can be calculated by taking the difference between the timestamps of the exit and entry.

Since we know when the bed exit occurs, we will segment the data into two parts. A truncated 5 to 10 minute window just before the exit will be created. This smaller portion of data is passed to stage 3 , the extraction of signal features. This smaller data range will decrease the processing time required to perform stage $3-6$, extracting the bed exit timings. 


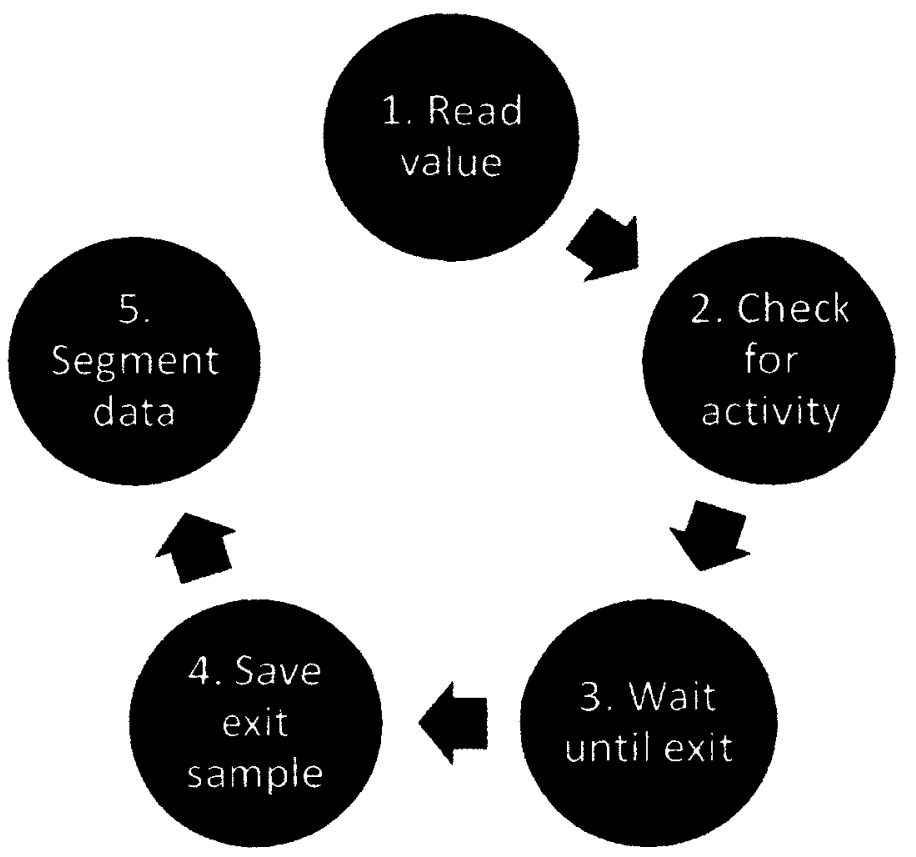

Figure 34: Flowchart for Step (2) Preprocessing data 


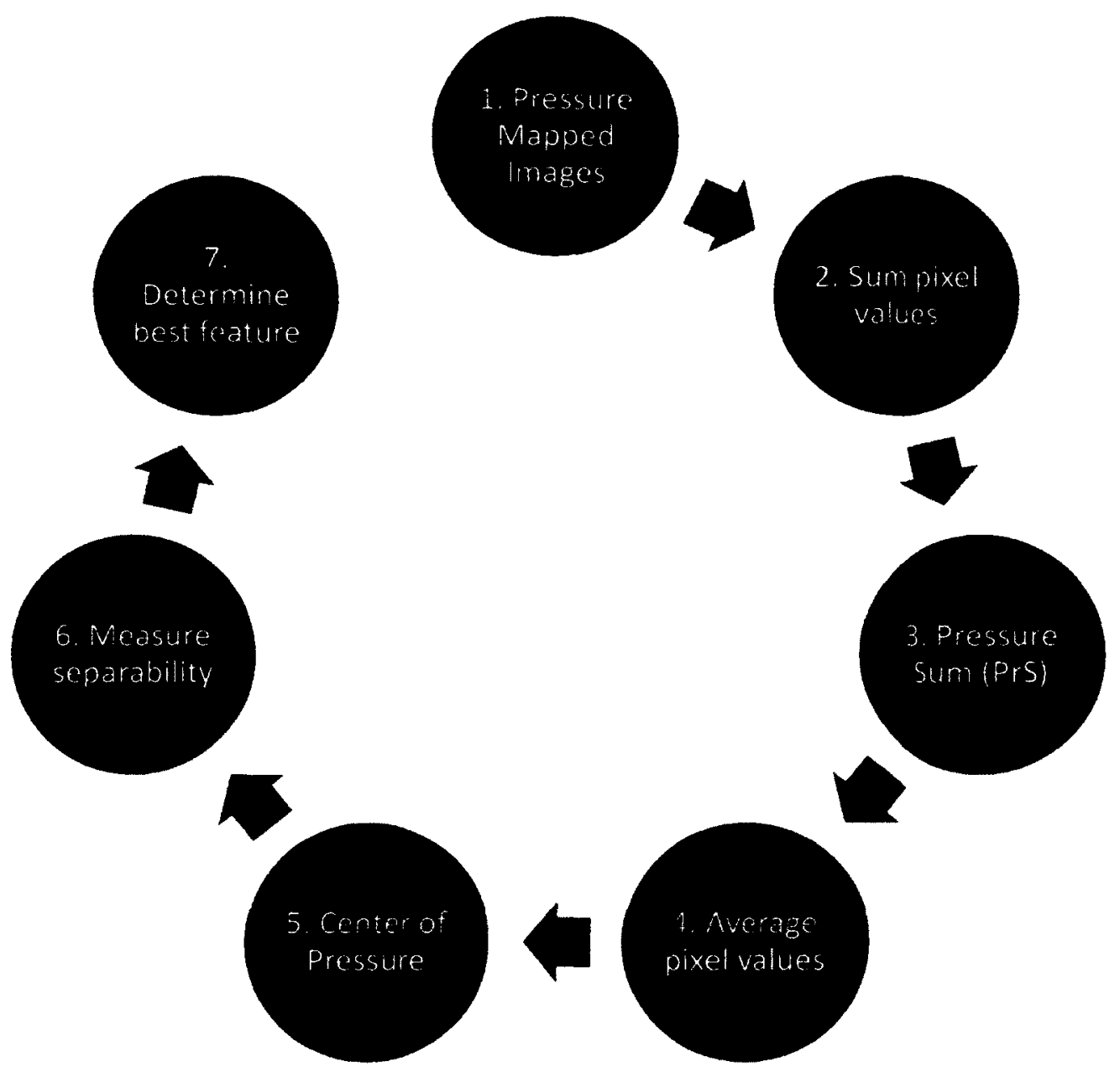

Figure 35: Flowchart of Step (3) Extracting features

The more complex clinical features including the lie-to-sit and sit-to-stand times can now be processed using the entry and exit information extracted using the first algorithm. To reduce the processing time required to calculate these features the recording where the bed exit occurs is truncated. The timestamp of the exit is loaded into the algorithm and a new file is created with a 5 or 10 minute window prior to the exit. This smaller file is then processed using the PrS, COP$x$, and COP-y feature extraction algorithm. 
Figure 35 summarizes the steps involved in stage 3, pressure mapped images are created, then the pixel values are summed giving the PrS. The pixel values are then averaged and the COP is calculated. Each of the extracted features is plotted and the lie-to-sit and sit-to-stand timings can be measured. This was done originally by manually selecting the point at which the lie-to-sit transfer occurred and ended based on visual inspection. The sit-to-stand transfer was determined the same way. An example of manually selected points on an exit is shown in Figure 37 . Because we have so many bed exits to process it would be helpful to automate the timing extraction process. Of the three features we can use to extract the timings, PrS, COP-x, COP-y, usually one has more easily differentiable states, the states being lying, sitting, and out as shown in Figure 36. In this example both COP-y and PrS have good separability in states. Once the best feature has been determined we move onto step 4, extracting clinical information.
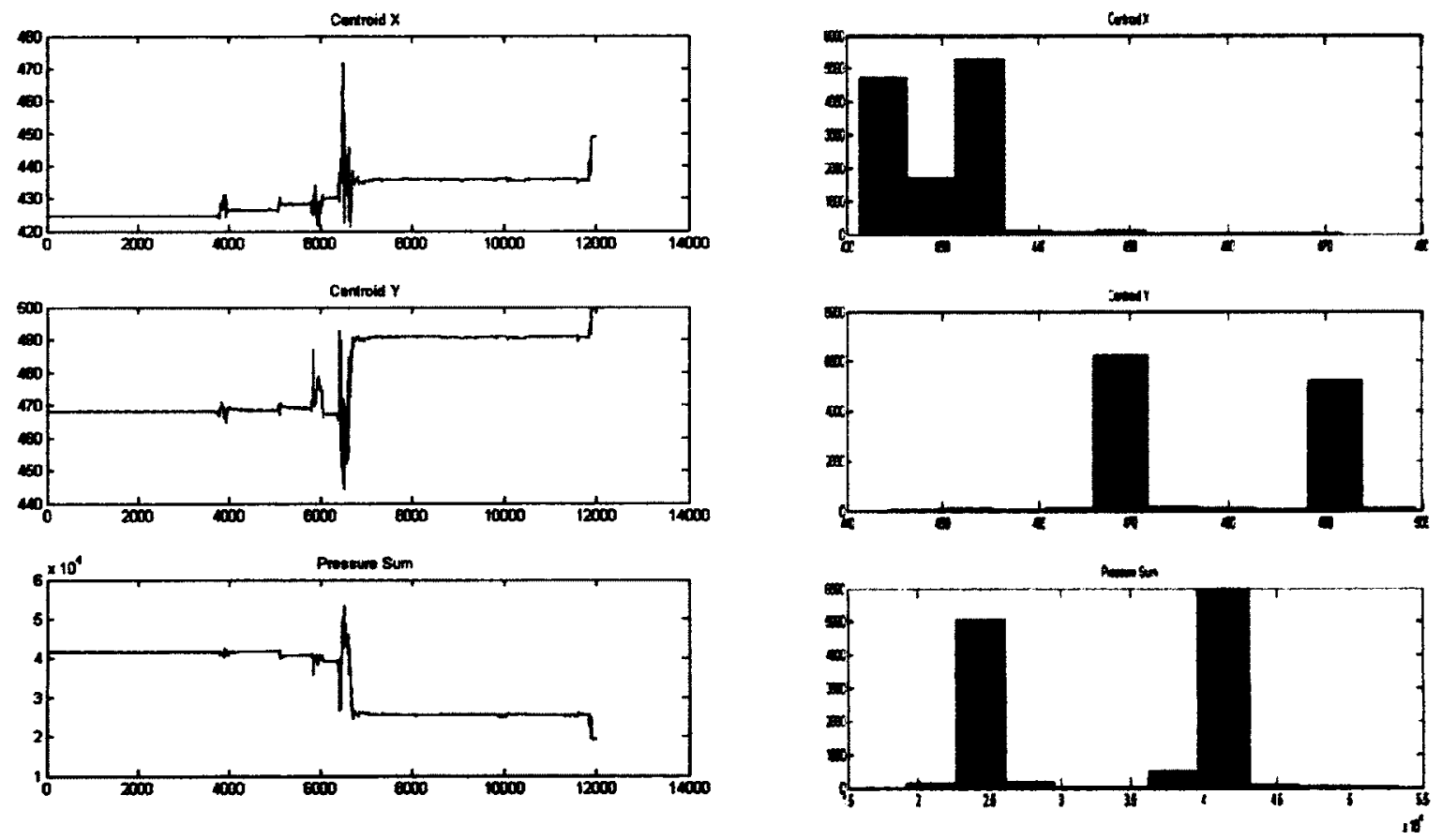

Figure 36: Probability distribution of features showing separability of states 


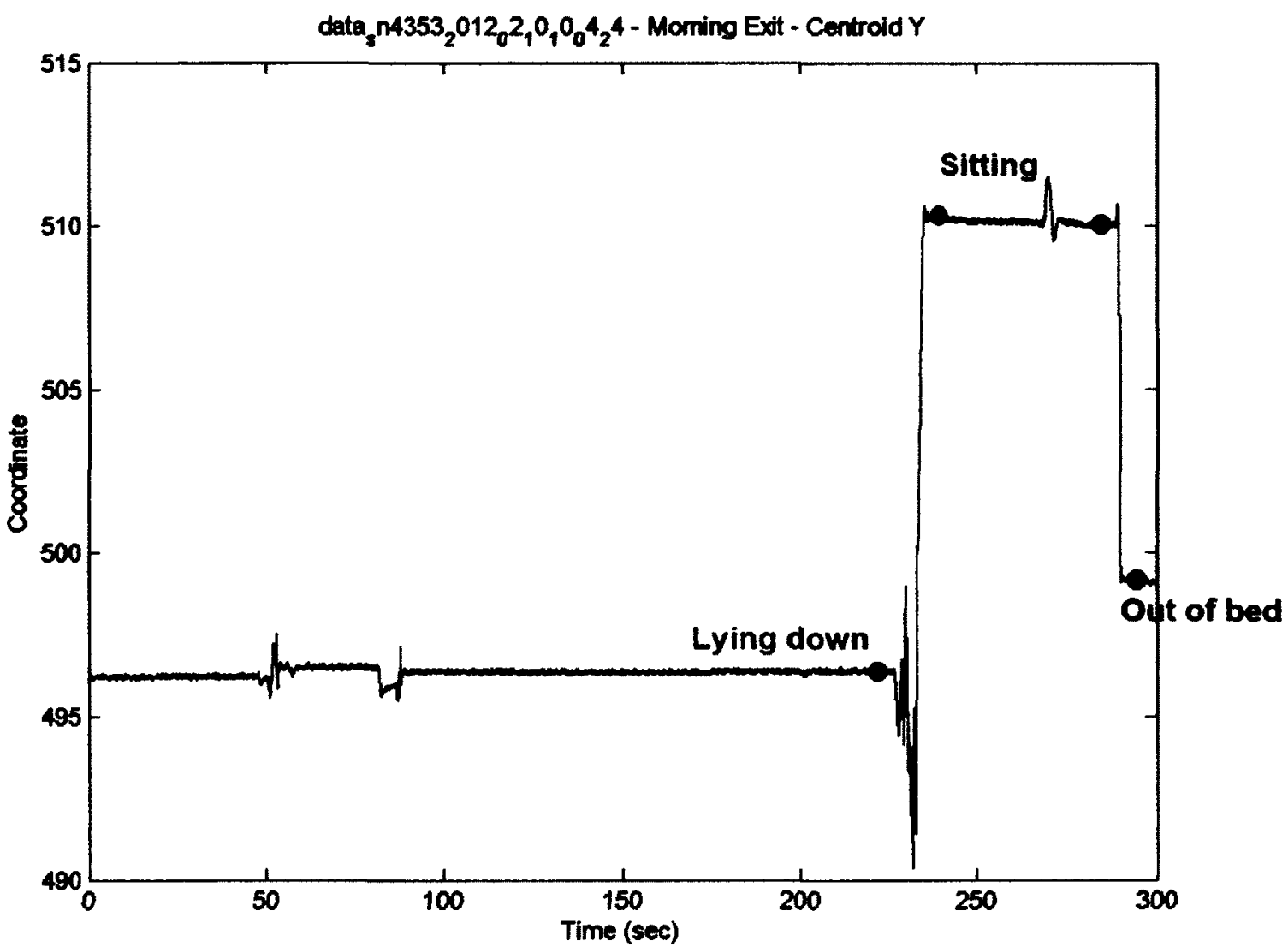

Figure 37: Stages of the bed exit. Manually selected

Using the bed exit data shown in Figure 37, we will explain the autonomous timing extraction algorithm. A flowchart was created to illustrate the processing required in step 4, extracting clinical information. The flowchart is shown in Figure 38. 


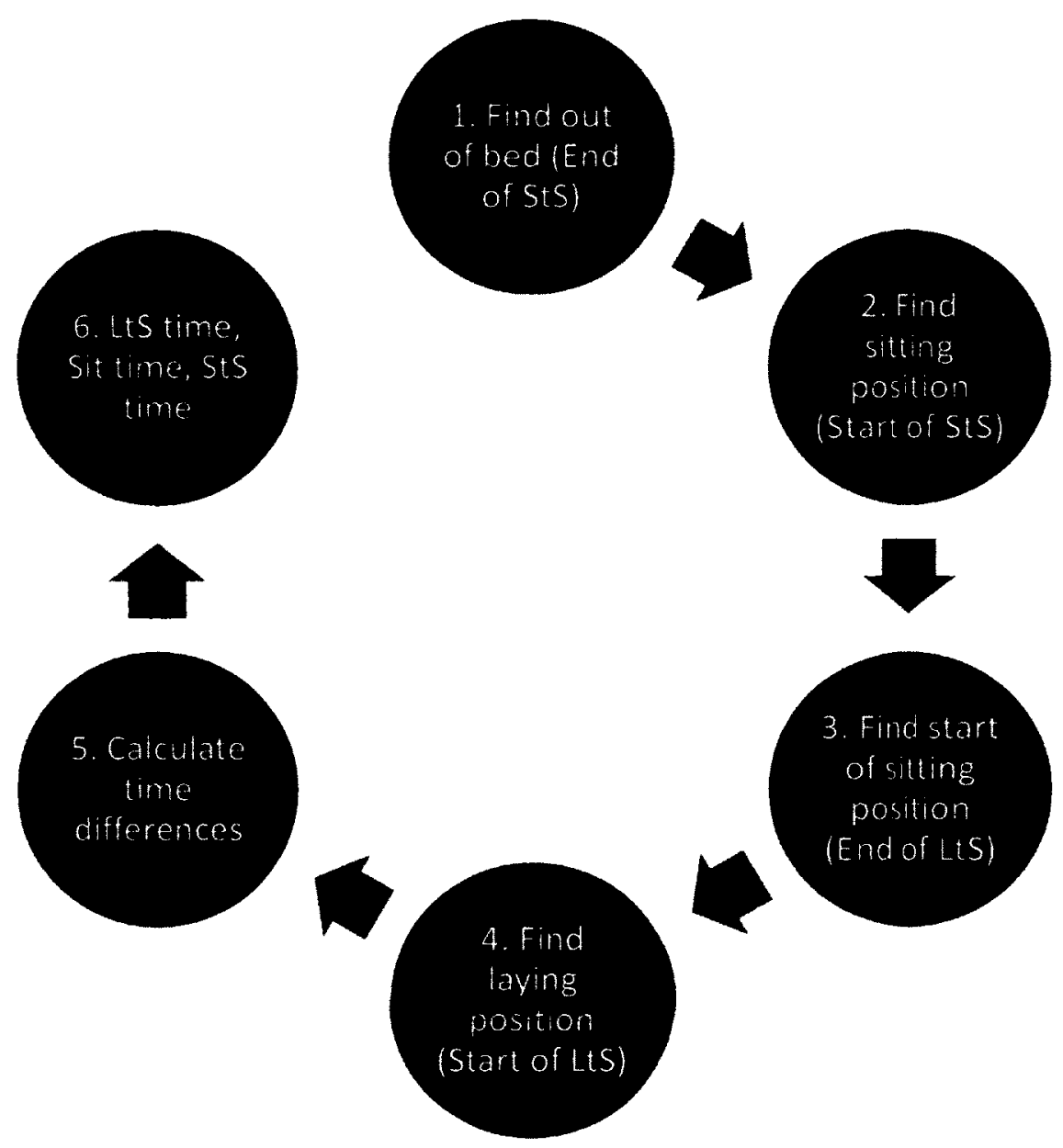

Figure 38: Flowchart of Step (4) Extracting clinical information from features

Using the truncated data, we know the first section of the data is when the patient is lying down. We need to determine when:

(1) Lying state end

(2) Sitting state begins

(3) Sitting state ends

(4) Out of bed 
We have access to the three features, PrS, COP-x, and COP-y. The simplest state to determine is (4) the out of bed state. The best feature for determining this is PrS, by simply using the same threshold that was used to determine bed occupancy for the full data set we will find the exact point that the patient is out of the bed. Using this point we can then work backwards to find the point when (3) the sitting state ends. This is shown in Figure 39. The out of bed state is annotated on both the PrS and COP-y state. COP-y was determined as the best feature for extracting the timings in this bed exit example.

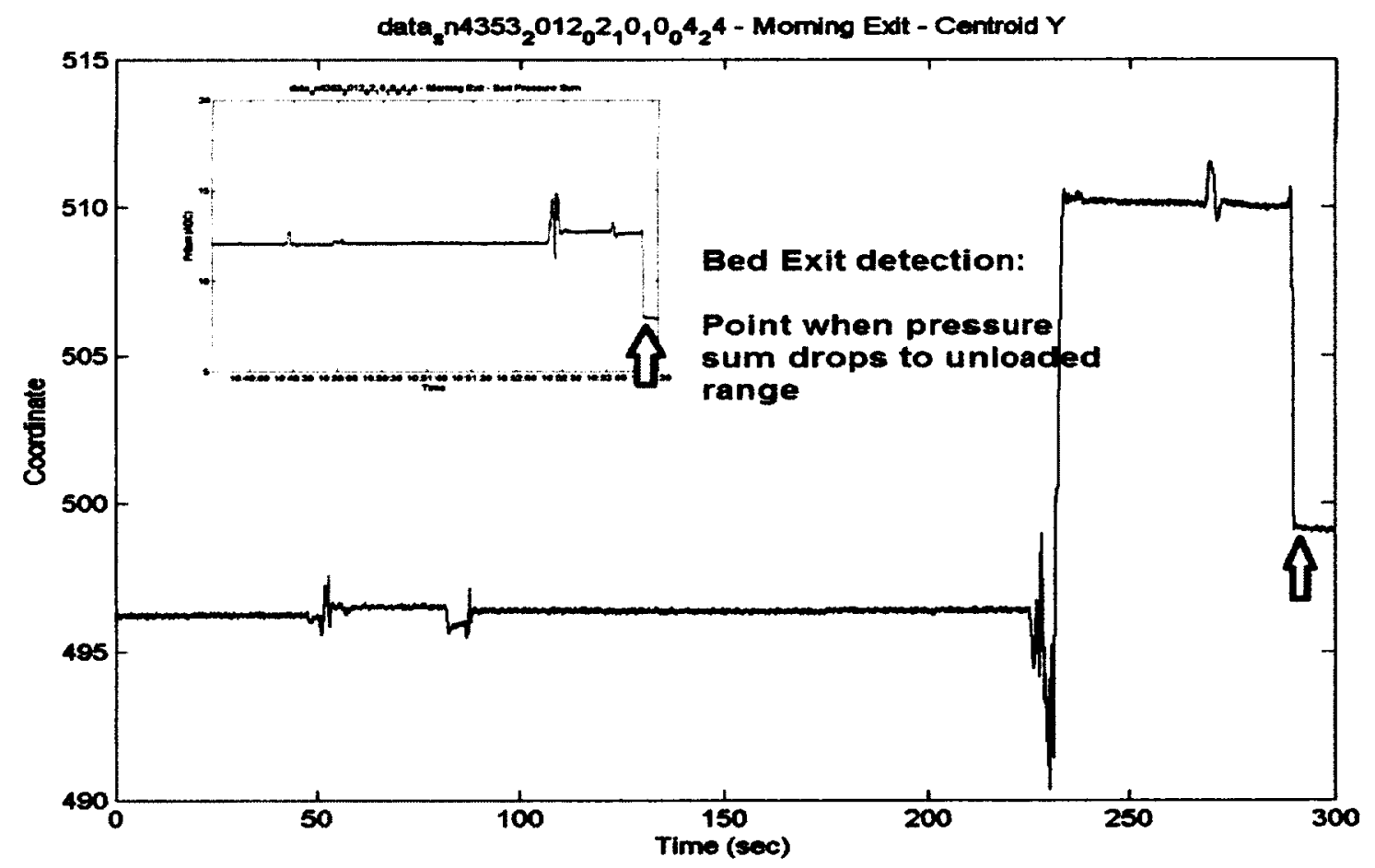

Figure 39: Extracting Timings: End of sit-to-stand 
As we look back from the exit point the waveform rises very steeply and then levels off. This portion of the data is the sitting state. The algorithm is designed to look back from the exit point and examine the COP-y value for stability. A threshold is set for what is defined as sitting in the COP-y feature. A value $>508$ could be used. The values above the threshold are analyzed for stability. We define stability as being with the normal range, which was configured as the average value with some buffer space of $+/-20 \%$ The values must stay within this range for a minimum of $1 \mathrm{sec}$ for the algorithm to continue, this is to ensure this transfer contains some portion of sit time. The algorithm looks for the last point the COP-y feature is within the normal range before it drops, this point would be end of the sit time and the starting point for the sit-tostand calculation. Figure $\mathbf{4 0}$ shows this progression in the algorithm.

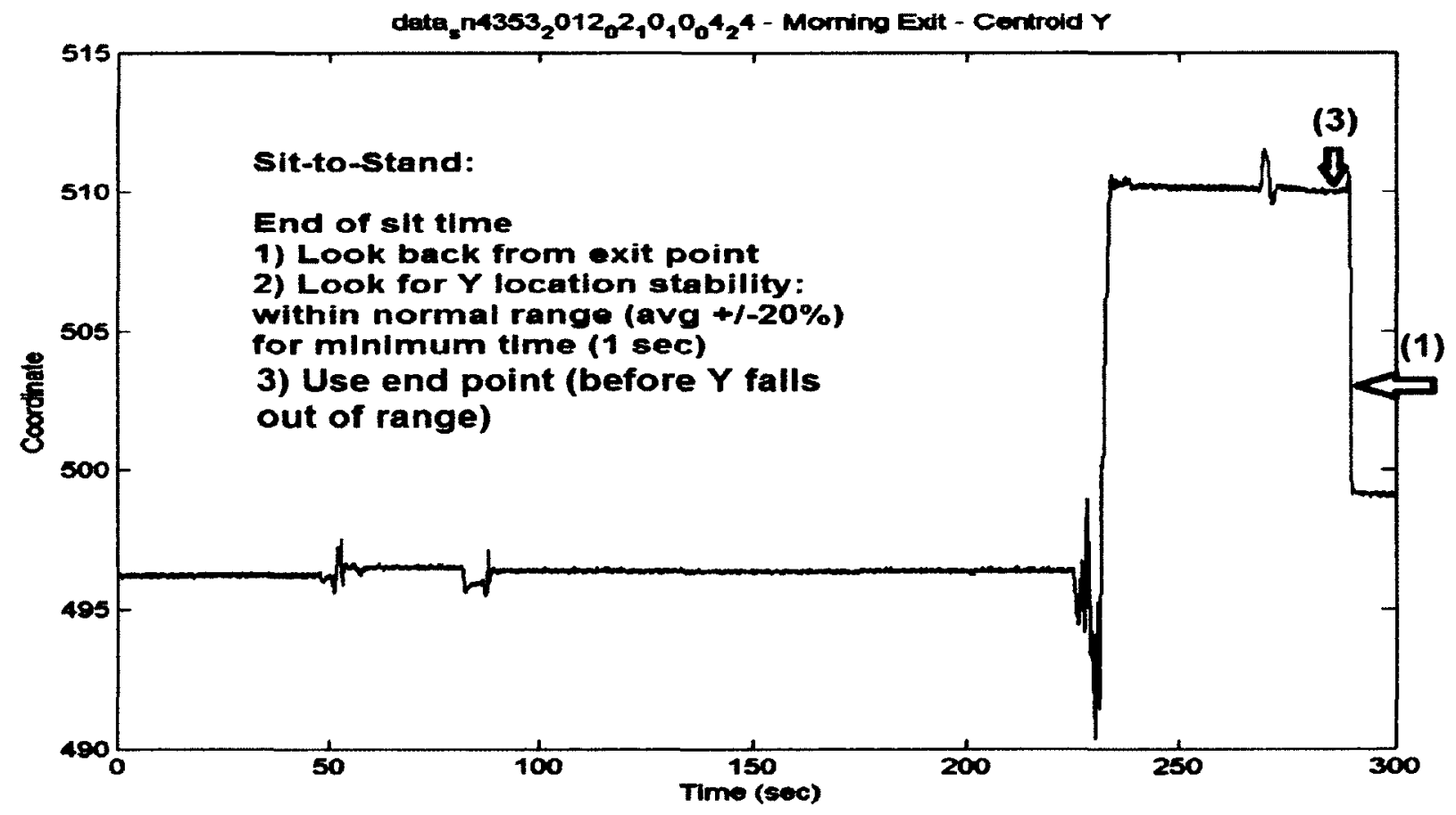

Figure 40: Extracting Timings: End of sit time (start of StS) 
We now have the start and end point for the sit-to-stand transfer, these points are saved and the time difference between them is calculated and exported in the StS column.

The next step is to determine when (2) sitting state begins. This is fairly simple to do, since we have already determined what the normal range for sitting is we look back from the sitting end point until the value moves out of the normal sit range into the lying range. We then look forward from the lying range to the first occurrence that the COP-y is in the normal sitting range. This is shown in Figure 41. This point is known as the start of the sit time.

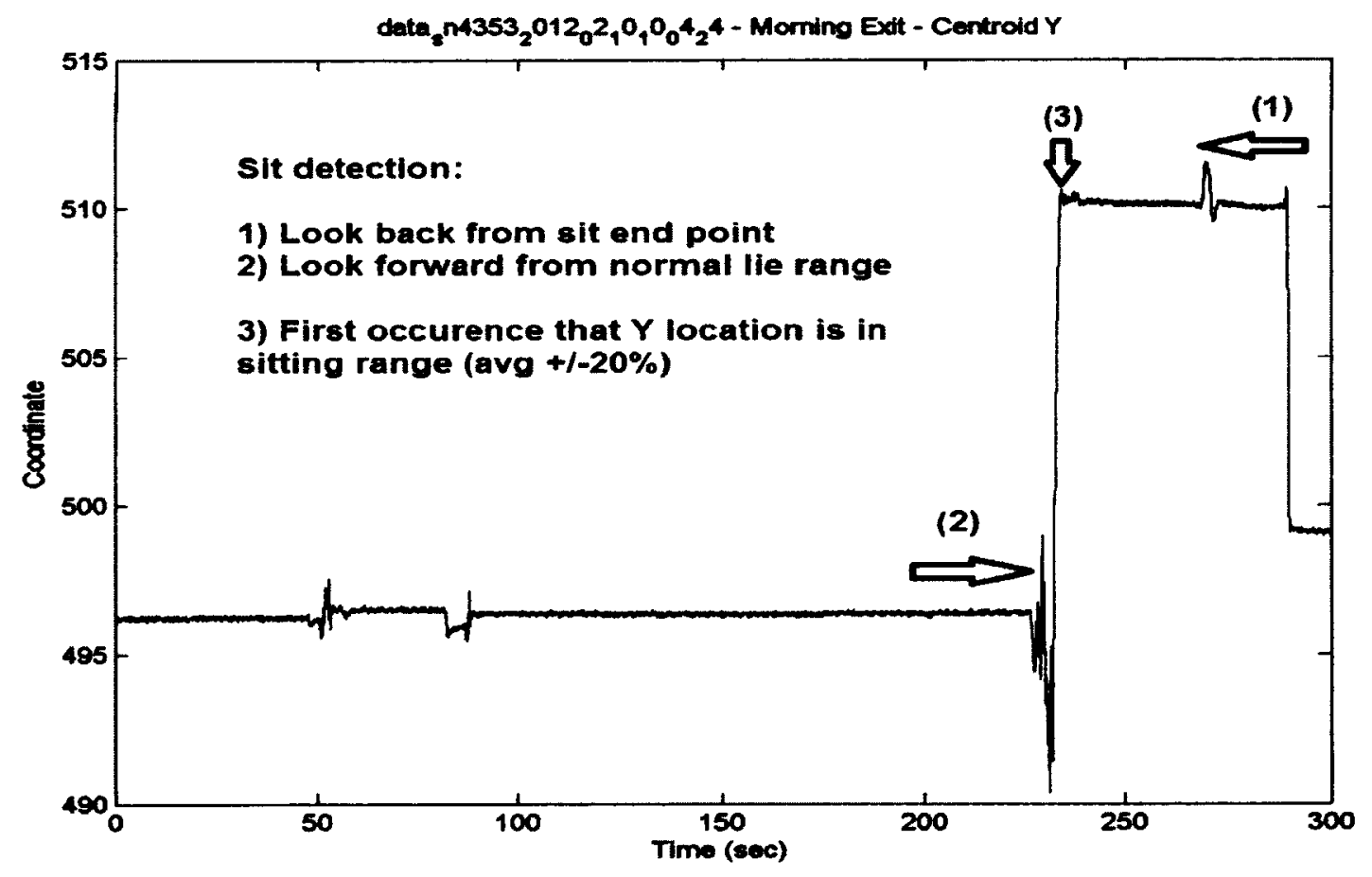

Figure 41: Extracting Timings: Start of sit time (end of LtS) 
Since we have both the start and end of the sitting phase we can calculate its duration and export this into the sit time column. We now need to determine the end of the lying phase in order to perform the lie-to-sit time calculation. Determining this is sometimes tricky because there is a lot of variability seen in the features during the lie-to-sit transfer, we need to ensure we capture the entire transfer to measure the time accurately. Therefore one of the rules we established was to look for a minimum time of stability in lying to ensure this was not part of the transfer. We begin by looking backwards from the beginning of the sit stage; once the COP-y value enters the normal lying range we examine the values for stability. We continue checking the values backwards and if they remain in the normal range for at least 5 seconds we can proceed with the next step. The next step is to look forward from the normal range to the last point of stability; this point is the end of stable lying and is the beginning of the lie-to-sit transfer. This is shown in Figure 42. The timestamp is saved and the lie-to-sit time is calculated by taking the difference between the end of lying timestamp and the start of sitting time stamp. These values are exported in to the lying timestamp and LtS columns. 


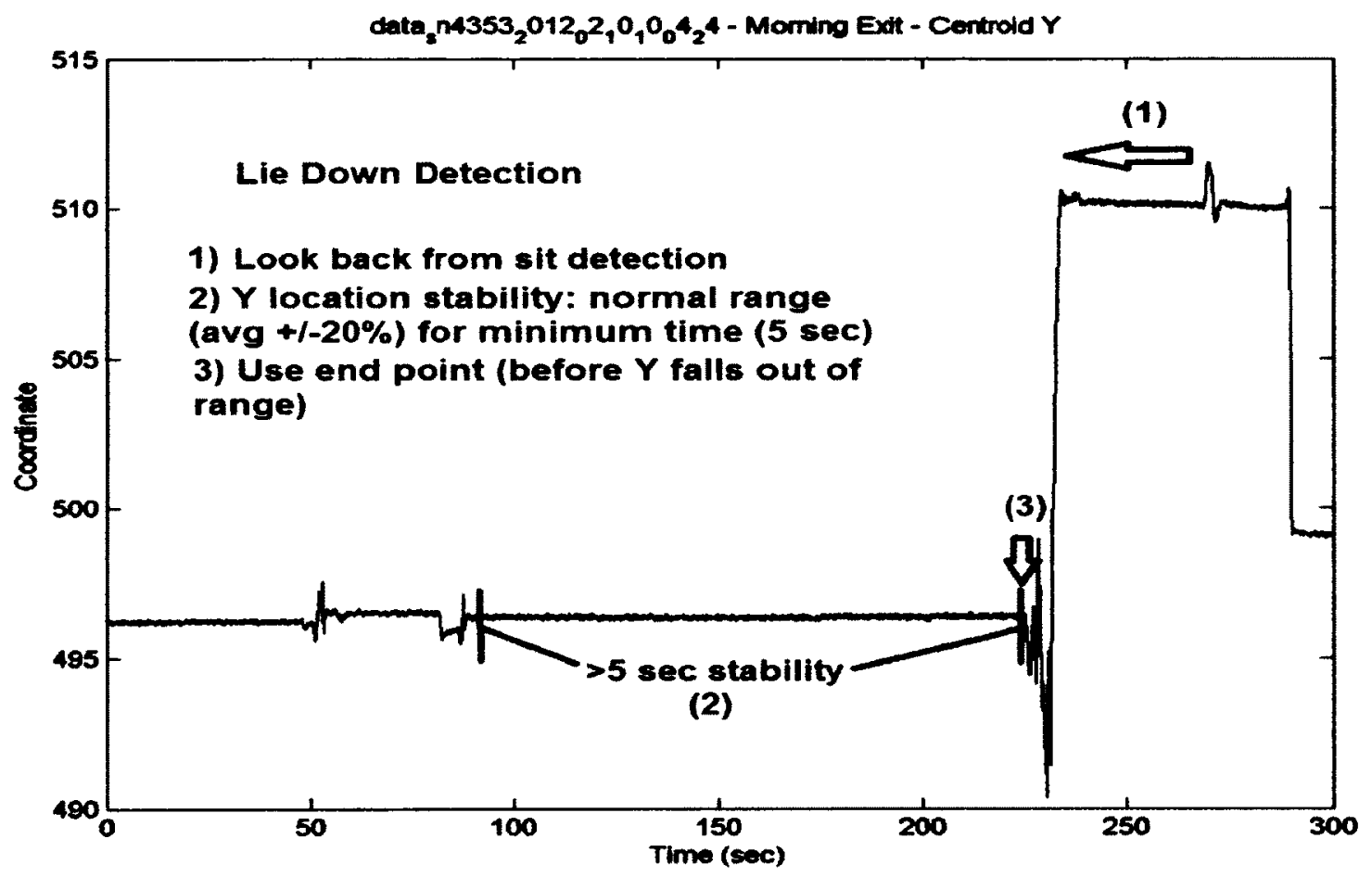

Figure 42: Extracting Timings: End of lie time (start of LtS)

Once the clinical information has been extracted the final stage is to output the processed data.

Several options for data output are available, which were discussed in section 4.2.4. To summarize these options, the clinical information can exported as values, plots, images, or videos. These options are configured from the main GUI, which will be discussed in 4.2.6.

\subsubsection{Feature comparison and performance}

A dataset containing one week of data, 7 bed exits from a real patient with the sensor installed in their home was processed using the automatic timing extraction algorithm. The four points required to calculate the LtS, sit time, and StS were automatically annotated on the plots. The plots and the timings were exported. 
The first exit was best extracted using the COP-x feature; it is shown in Figure 43.

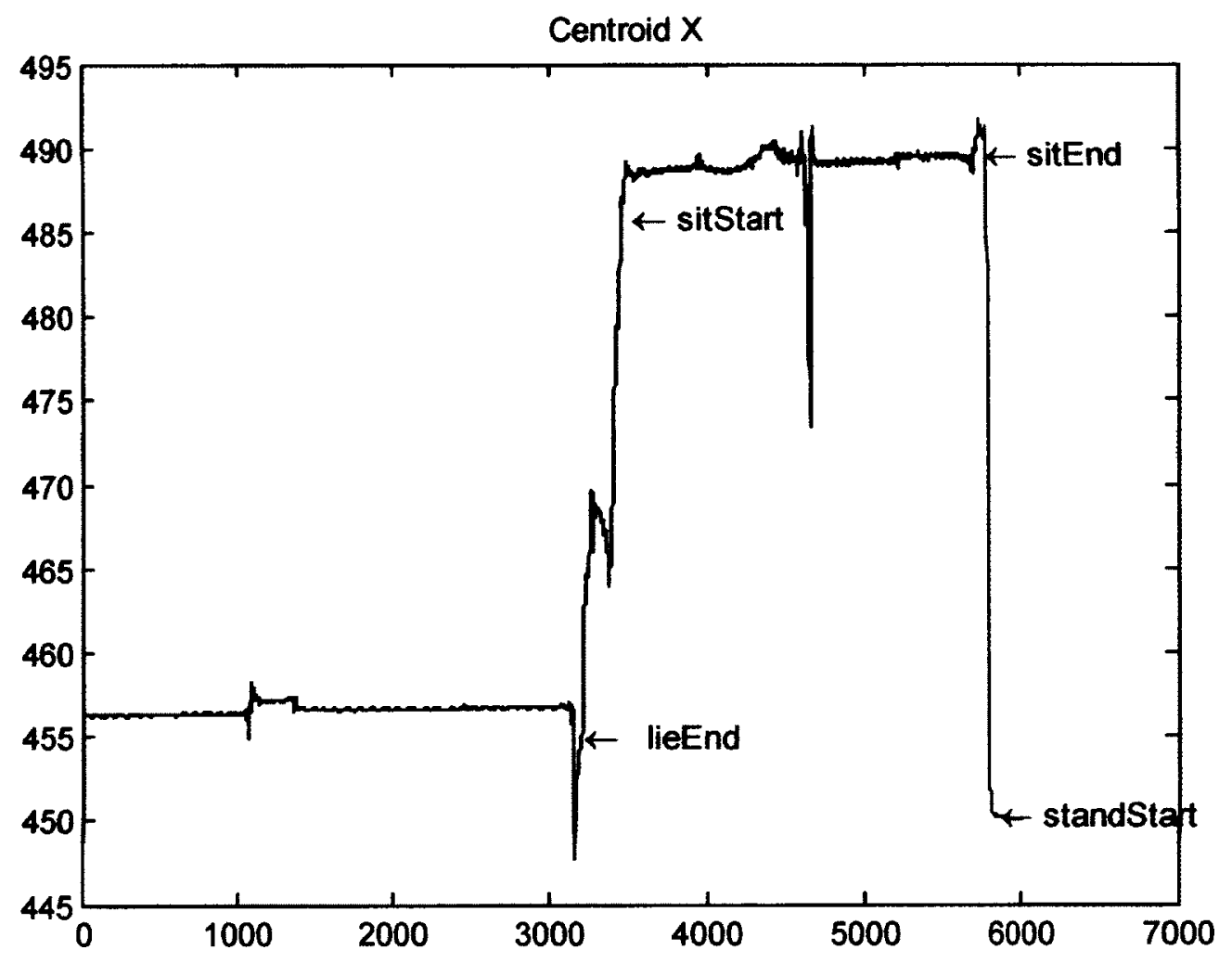

Figure 43: Bed exit 1 using automatic timing extraction. data_sn4352_2011_12_09_01_18_57

The extracted timings are shown in table 8.

Table 10: Bed exit 1 extracted timings

\begin{tabular}{|l|l|l|}
\hline LieToSit (s) & SitDuration (s) & SitToStand (s) \\
\hline 15.5500 & 111.6000 & 5.4500 \\
\hline
\end{tabular}


The second exit was best extracted using the COP-y feature; it is shown in Figure 44.

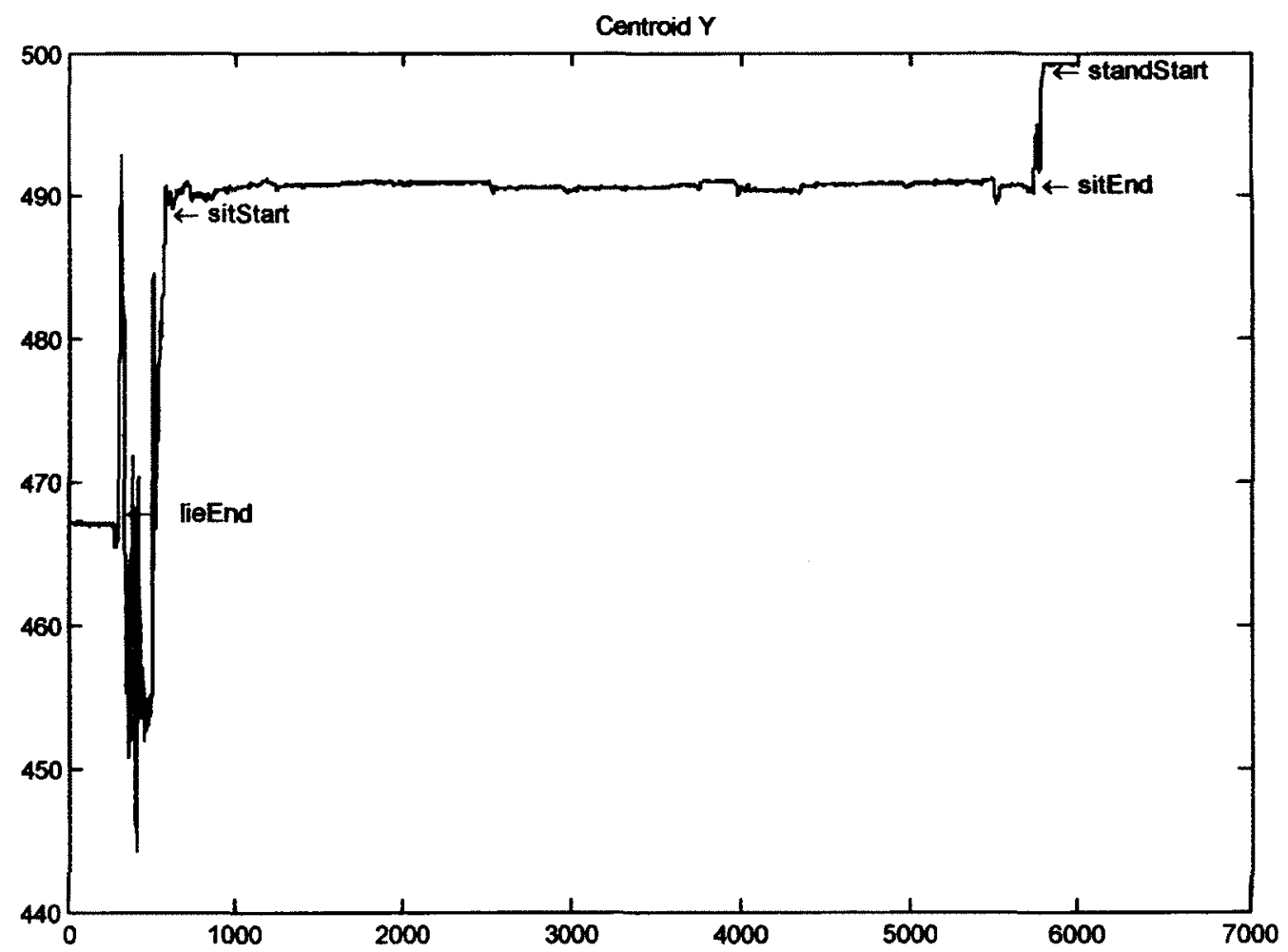

Figure 44: Bed exit 2 using automatic timing extraction. data_sn4352_2011_12_10_10_19_30

The extracted timings are shown in table 9.

Table 11: Bed exit 2 extracted timings

\begin{tabular}{|l|l|l|}
\hline LieToSit (s) & SitDuration (s) & SitToStand (s) \\
\hline 13.9500 & 258.7000 & 2.6000 \\
\hline
\end{tabular}


The third exit was best extracted using the COP-x feature; it is shown in Figure 45 .

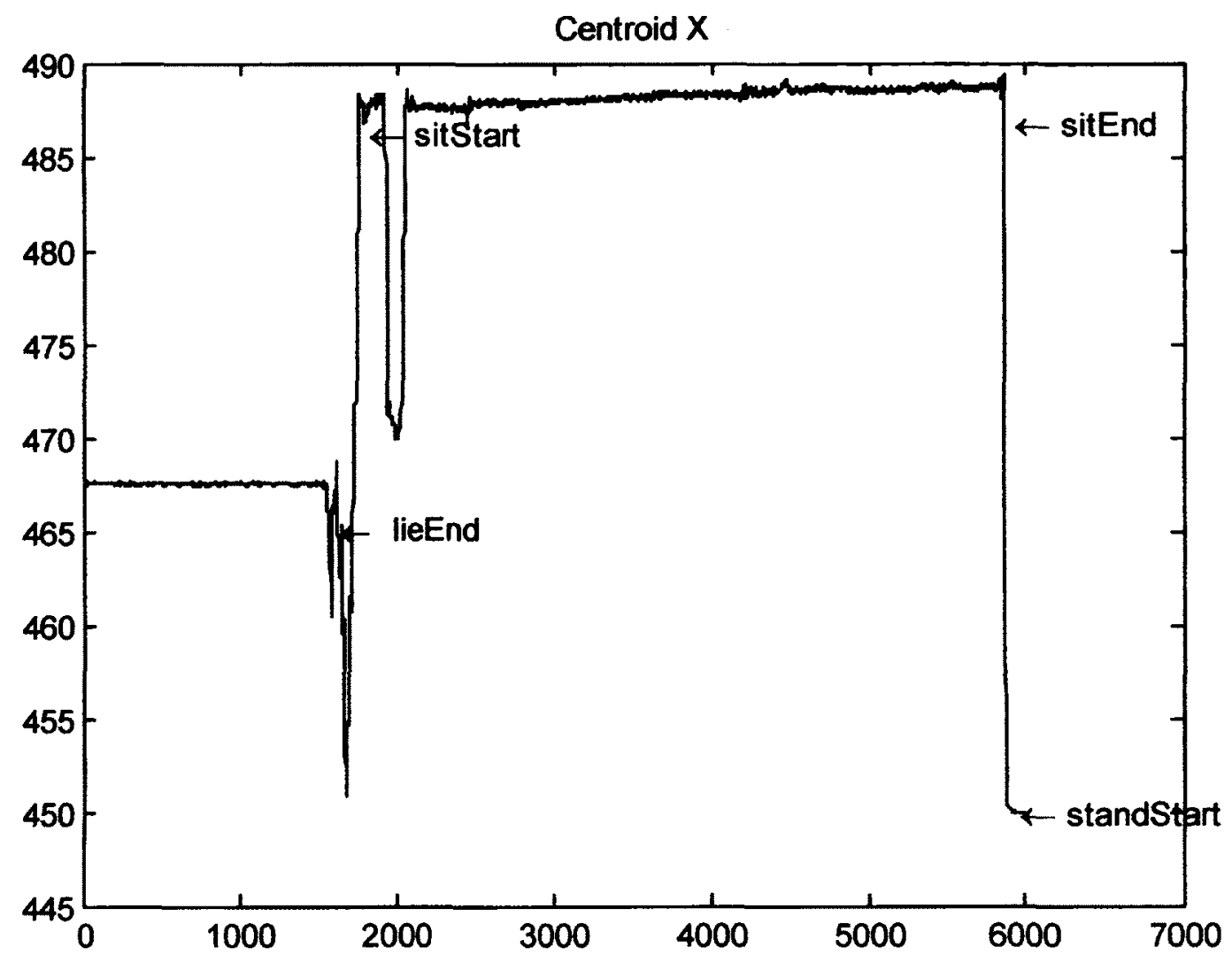

Figure 45: Bed exit 3 using automatic timing extraction. data_sn4352_2011_12_10_13_19_33

The extracted timings are shown in table 10.

Table 12: Bed exit 3 extracted timings

\begin{tabular}{|l|l|l|}
\hline LieToSit (s) & SitDuration (s) & SitToStand (s) \\
\hline 9.6000 & 205.5000 & 1.6000 \\
\hline
\end{tabular}


The fourth exit was best extracted using the COP-x feature; it is shown in Figure 46.

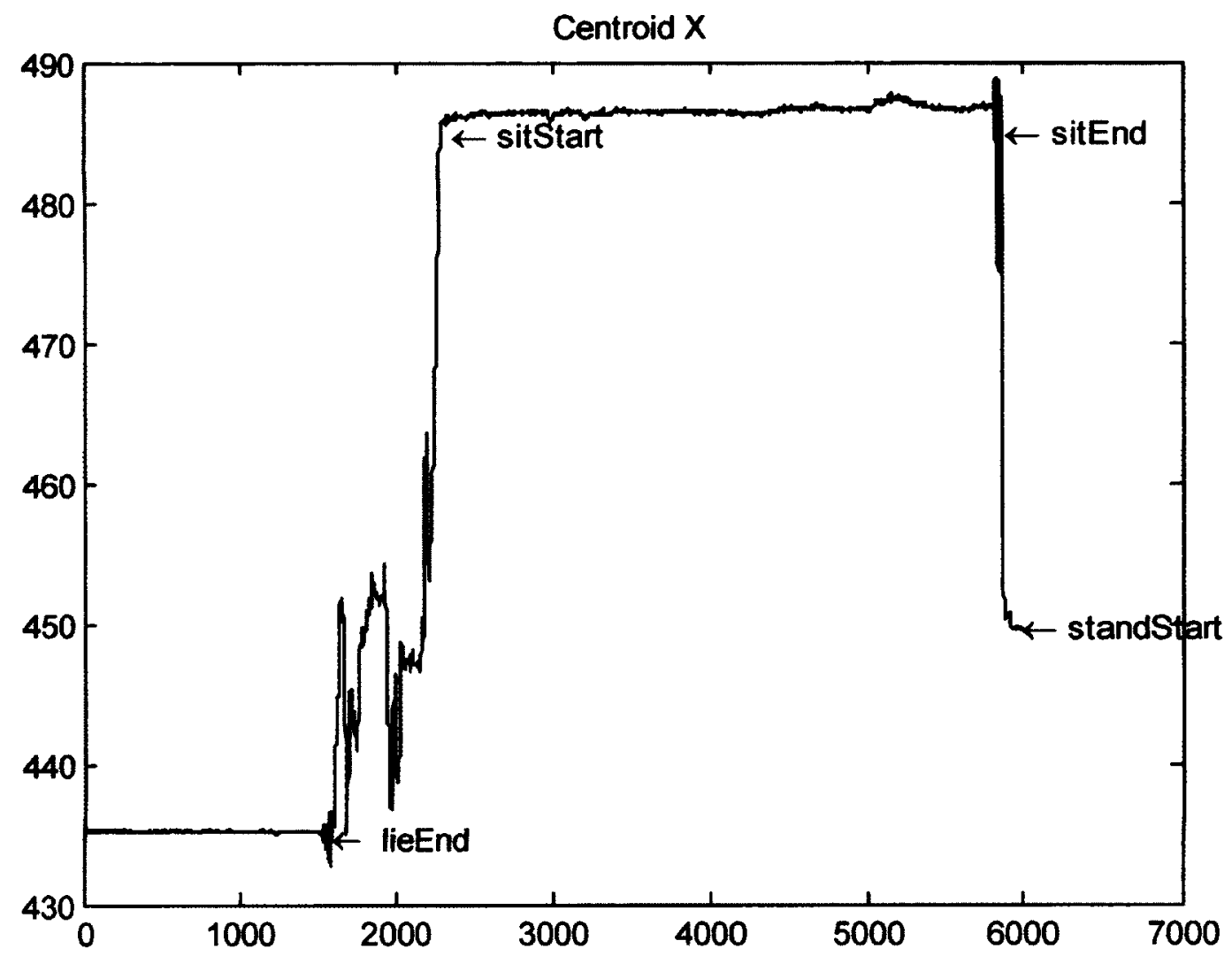

Figure 46: Bed exit 4 using automatic timing extraction. data_sn4352_2011_12_11_09_19_53

The extracted timings are shown in table 11.

Table 13: Bed exit 4 extracted timings

\begin{tabular}{|l|l|l|}
\hline LieToSit (s) & SitDuration (s) & SitToStand (s) \\
\hline 38.4500 & 176.5500 & 4.9500 \\
\hline
\end{tabular}


The fifth exit was best extracted using the COP-x feature; it is shown in Figure 47.

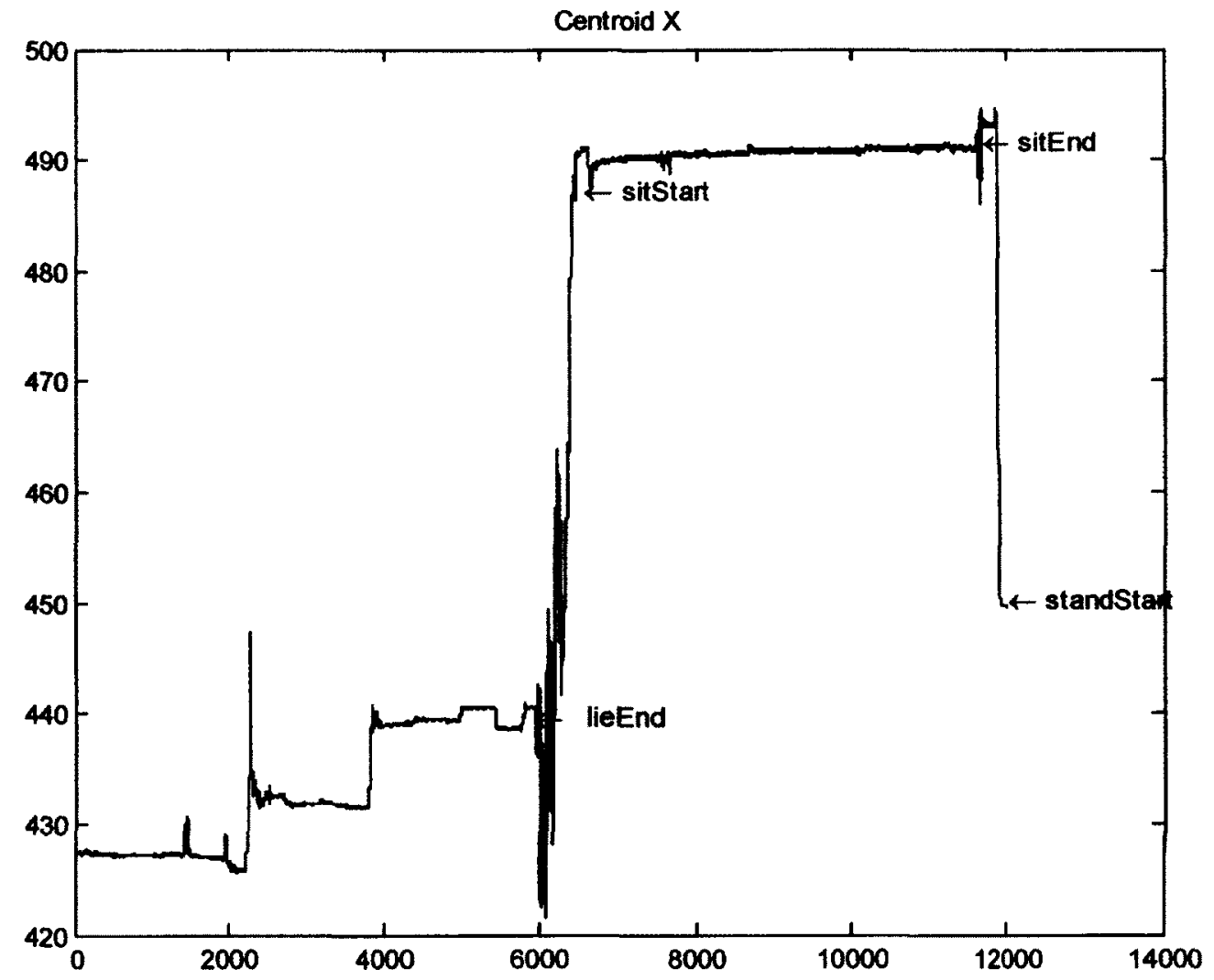

Figure 47: Bed exit 5 using automatic timing extraction. data_sn4352_2011_12_12_07_20_15

The extracted timings are shown in table 12 .

Table 14: Bed exit 5 extracted timings

\begin{tabular}{|l|l|l|}
\hline LieToSit (s) & SitDuration (s) & SitToStand (s) \\
\hline 32.1500 & 257.1500 & 16.3000 \\
\hline
\end{tabular}


The sixth exit was best extracted using the COP-x feature; it is shown in Figure 48.

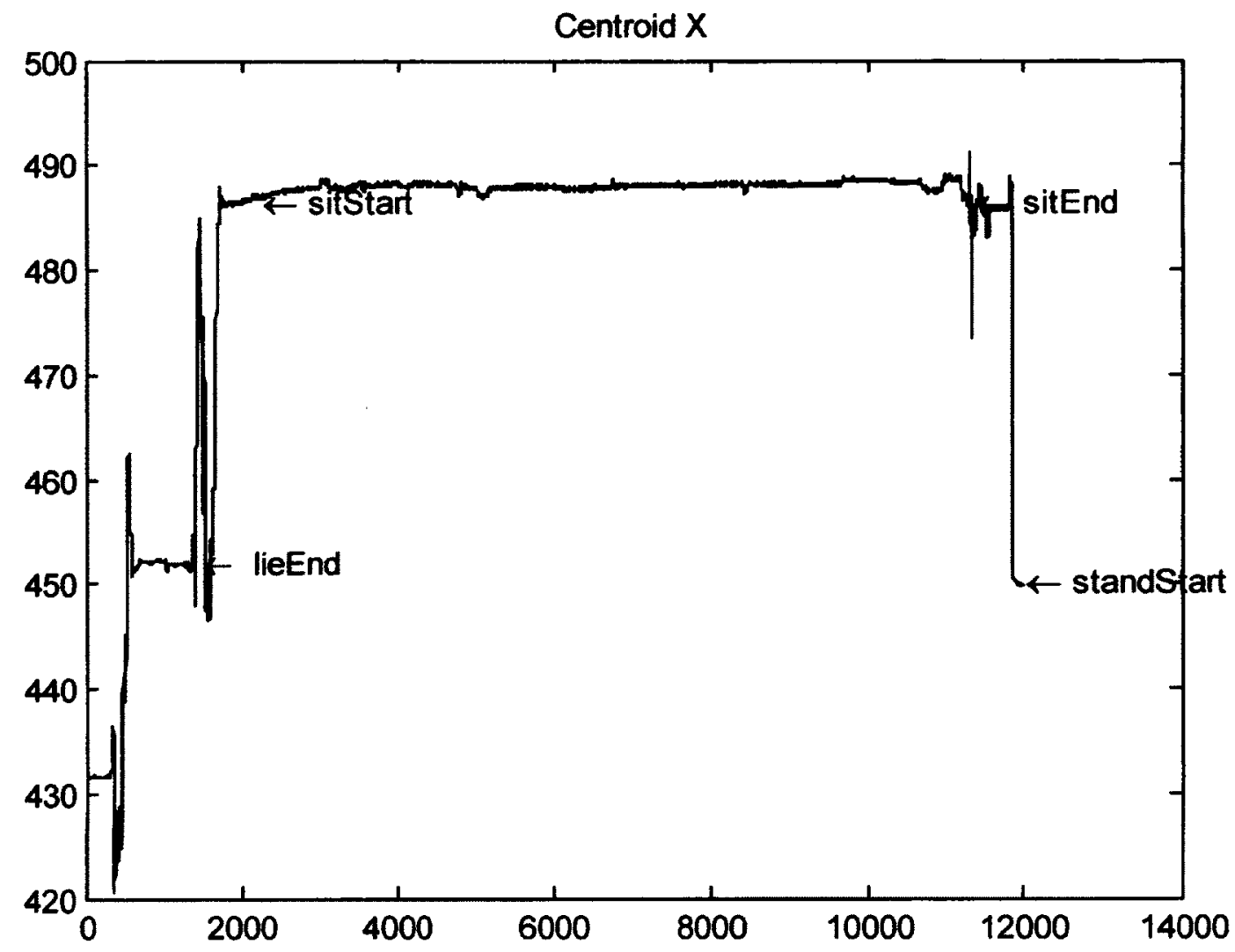

Figure 48: Bed exit 6 using automatic timing extraction. data_sn4352_2011_12_13_06_20_38

The extracted timings are shown in table 13.

Table 15: Bed exit 6 extracted timings

\begin{tabular}{|l|l|l|}
\hline LieToSit (s) & SitDuration (s) & SitToStand (s) \\
\hline 38.6000 & 458.9500 & 29.5500 \\
\hline
\end{tabular}


The seventh exit was best extracted using the COP-x feature; it is shown in Figure 49.

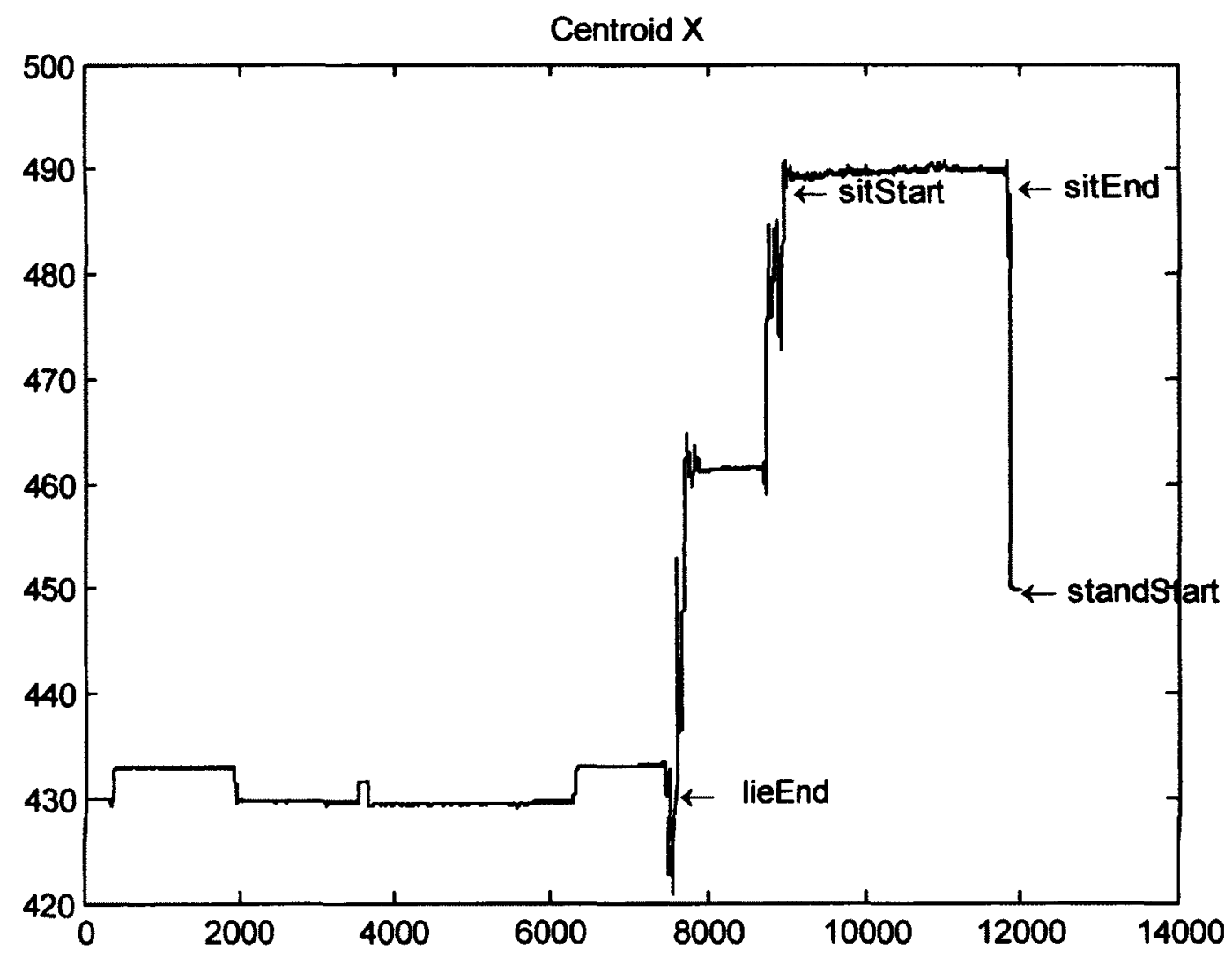

Figure 49: Bed exit 7 using automatic timing extraction. data_sn4352_2011_12_13_11_20_43

The extracted timings are shown in table 14.

Table 16: Bed exit 7 extracted timings

\begin{tabular}{|l|l|l|}
\hline LieToSit (s) & SitDuration (s) & SitToStand (s) \\
\hline 73.5000 & 144.9000 & 1.9500 \\
\hline
\end{tabular}


From the 7 bed exits, the best feature for extracting the timings was the COP-x. It was used to extract 6 or 7 bed exit timings. Generally the participants will exit the bed in the same manor every time; this section's purpose was to show the similarities in bed exits from an engineering perspective. Chapter 5 will examine the bed exits timings in a medical view.

\subsubsection{Design of GUI}

Two graphical user interfaces (GUIs) were developed for processing the bed mat data. The first GUI called Bed Sensor Research Tool Beta 1 is shown in Figure 50. This tool was developed for use by non-technical researchers. It features a simple interface with limited functions. The purpose of this software is to be able to generate plots over a full range or custom range of a recording file with the option of displaying any or all of the four features, PrS (real time), PrS, COP-x, COP-y. The software can also generate pressure mapped images or pressure playback videos to assist researchers in determining the patient's position in the bed. An option for smooth filtering was developed which uses bicubic interpolation in the image / video creation process to generate a more realistic appearance of pressure on the mat. Bicubic interpolation was found to be far superior to other interpolation methods such as bilinear interpolation or nearest neighbour. Bicubic uses 16 pixels (4x4), where bilinear uses the 4 pixels $(2 \times 2)$ that surround a given pixel. The intensity and location of the pixels are used to determine the value for the interpolated pixel. Resampling the images using the bicubic method created smoother pressure mapped images.

Using this tool, researchers are able to confirm the lie-to-sit and sit-to-stand transfers by using the pressure playback videos or images. 


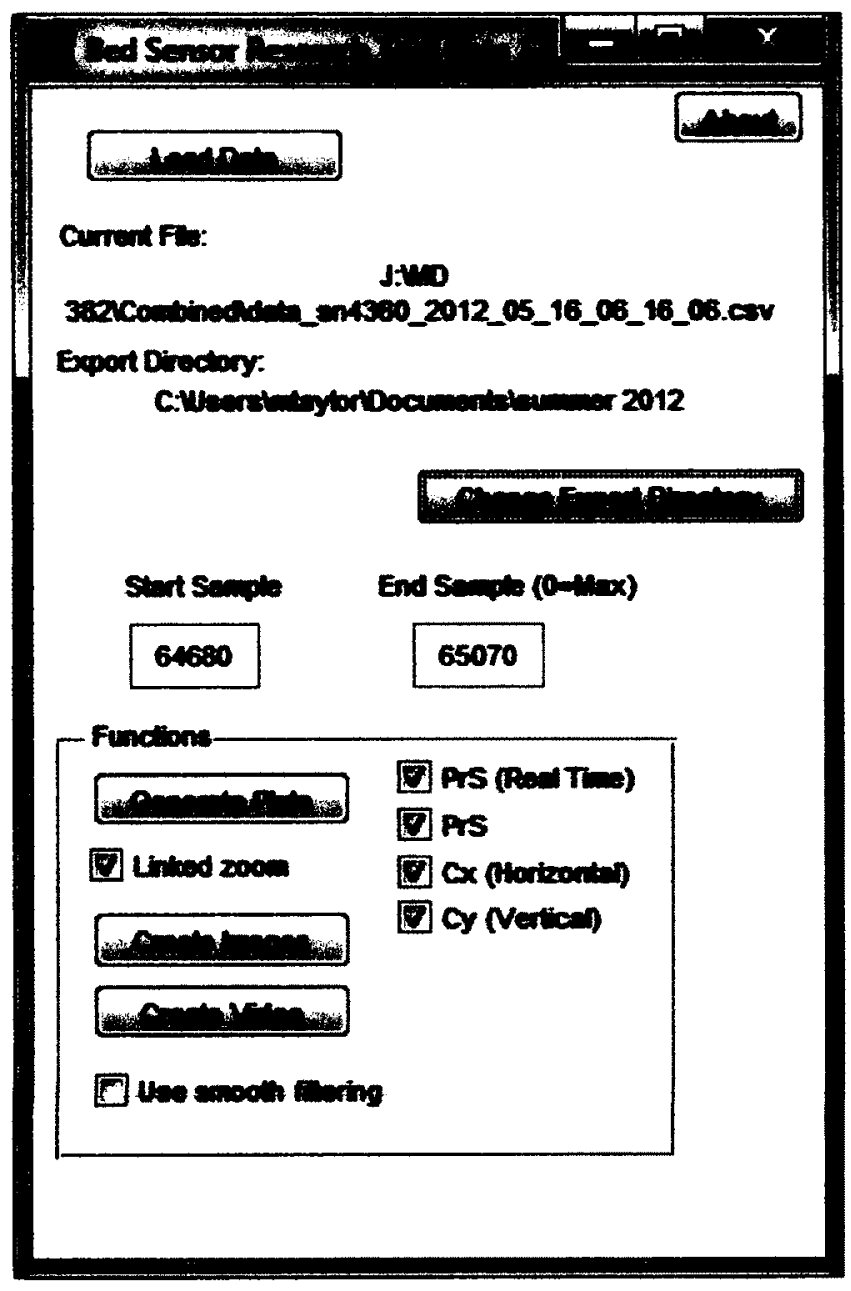

Figure 50: Bed Sensor Research Tool Beta 1

The other GUI developed for the bed mat data is the main control center for processing. This software is reserved for technical researchers use only as it requires specialized knowledge to operate. The GUI is shown in Figure 51. This GUI has access to a wide array of scripts developed for the bed mat data. Some of the functions are technical in nature for extracting features and clinical information. Other functions are for organizing data and checking its integrity. We will go through all the functions available in this tool. 
Pressure recordings are loaded by clicking "Select data"; they are listed in the box beside it. By clicking "Sort data", the recording files will be listed in chronological order. This function was necessary to develop because the files would otherwise be in alphabetical order, which created problems when the pressure sensor mat was replaced because of mechanical failure, the file naming scheme would change.

The next function is "Check data", this function scans through all the loaded recordings and checks for discontinuity. We know that each file should be 1 hour in duration, so this script checks the time between the files. When a discontinuity is found, usually due to a power failure, the file is flagged. Then the operator clicks "Separate data", this places all the continuous recording files into separate folders, this allows us to process all valid recording files in a batch and skip the invalid ones.

Since we have experienced problems with the pressure mat equipment failing we sometimes need to perform other tests on the data. We have seen that when a mat fails, sometimes the sensor values report abnormally high readings, even when in the unloaded state. Using the check sensors script, we configure the upper and lower limit to define the normal range for unloaded sensors. We select a recording file during the day, when we expect no bed occupancy, all the sensor values are scanned and a counter keeps track of how often each of the sensors reports an out of range value. The plot is then generated and displayed within the main control center GUI. This check can determine if specific sensors have failed or if the entire mat is no longer working properly. Similarly, a night recording could be used, when we expect some bed occupancy, if we don't see sensors out of the unloaded range, we know the mat is no longer working properly. 


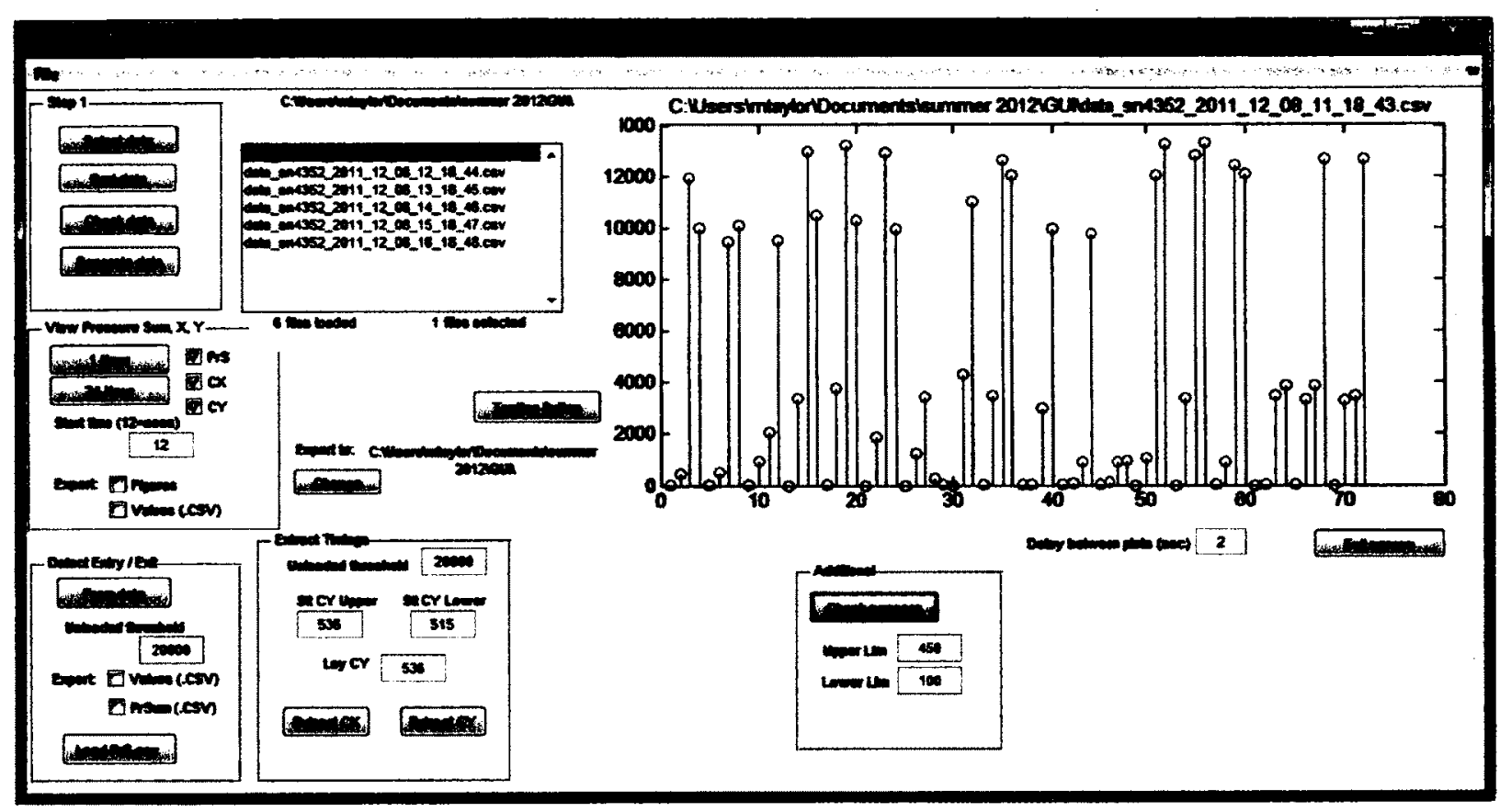

Figure 51: Bed Sensor Main Control Center

There are 3 groups of functions for extracting features and clinical information. The first group are for feature extraction, there are options for extracting PrS, COP-x, COP-y and exporting these as figures or as values into a CSV file. The figures can be created as one per file ( 1 hour) or one figure for 24 hours. The start time defines the 24 hour period and is usually 12 noon to 12 noon the following day, to capture the full night of data. The second group is to detect bed entries and exits; this calculation was explained earlier and basically works by comparing the PrS to the unloaded threshold. When entries, exits, no activity, and continuous activity are detected for the loaded data, a CSV file is exported giving the timestamps of each bed entry and exit. The next group of functions are for the bed exit timings extraction algorithm. Once the operator has set the normal range for the patient's positions, this includes setting the upper and lower limits for sitting and lying in the COP-x or COP-y feature, they can select to extract the timings either 
using COP-x or COP-y methods. Plots are generated and the extracted LtS, sit duration, StS times are exported.

The GUI allows us to process a large batch of recordings without any user effort after setting the required threshold values and options. This is the ideal way to deal with 100s of hours of recordings. 


\section{Chapter 5: Results}

\subsection{Floor Sensor Results}

We assessed the standing stability of an older adult using the processing algorithms discussed in section 4.1. The floor sensor was installed at the Bruyère Continuing Care facility and data were collected as part of an ongoing clinical intervention trial. With the consent of an older adult with chronic stroke, ten 60 -second recordings were made of quiet standing and lateral weight shifting over a two week period. Data were collected from a healthy young male and served as a comparison dataset. 


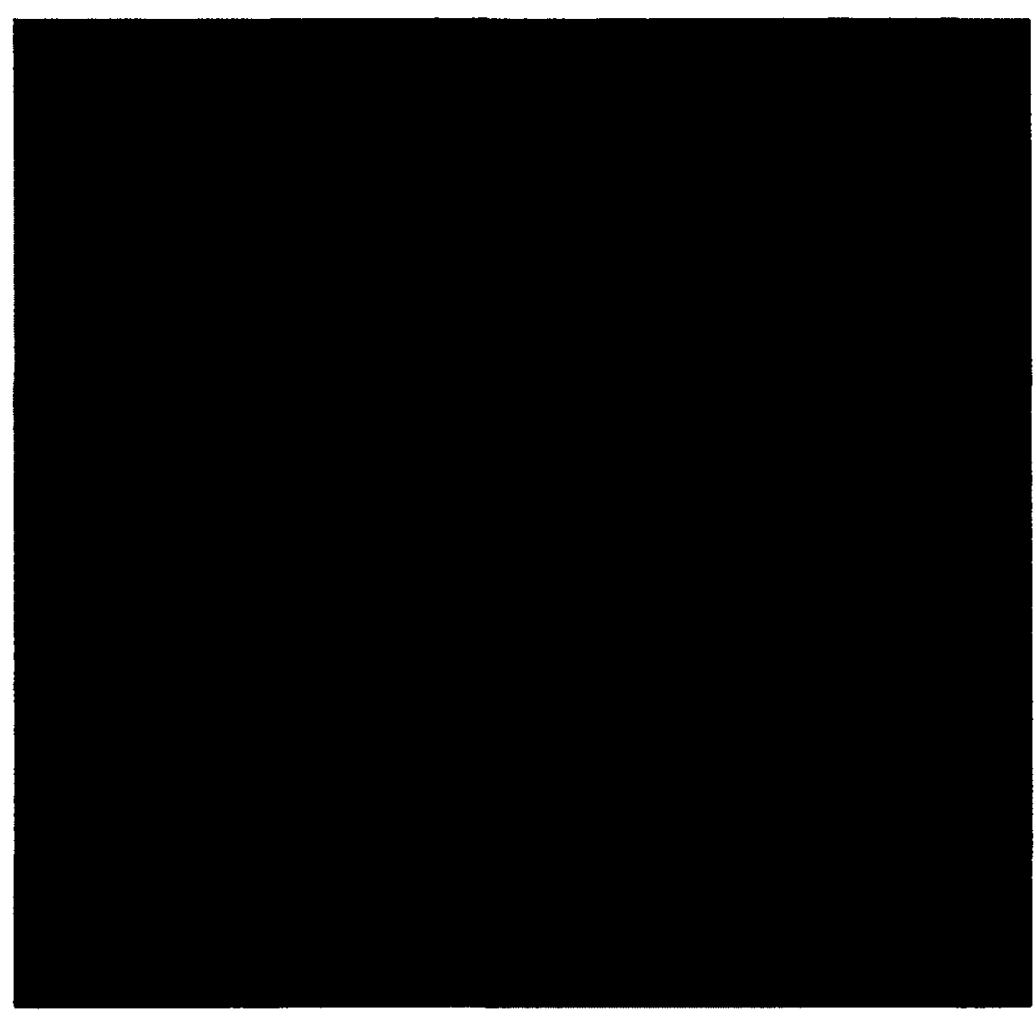

Figure 52: Young adult standing on sensor array with balanced pressure under each foot and symmetrical stance

The first three clinical features: lean ratio (amount of lean), footprint symmetry (asymmetry in weight bearing), and sway area were extracted from the quiet stance recordings. The fourth feature, movement velocity (dynamic balance), speed at which they can shift their weight is extracted from the lateral weight shifting recording. From each 60 second recording 200 samples are generated, one every $300 \mathrm{msec}$. The amount of lean is calculated by averaging the amount of pressure seen under each foot over the 200 samples. The pressure distribution under each foot is observed from the image output as well as the COP locations, an example of which was shown in Figure 52. The amount of sway is observed by plotting the COP locations over time, as shown in Figure 53 and Figure 54. The total area in which the COP has varied is also important as it may 
be useful in comparing between trials or participants. The speed at which the COP moves is calculated by averaging the speed over the 200 samples generated from the second recording.
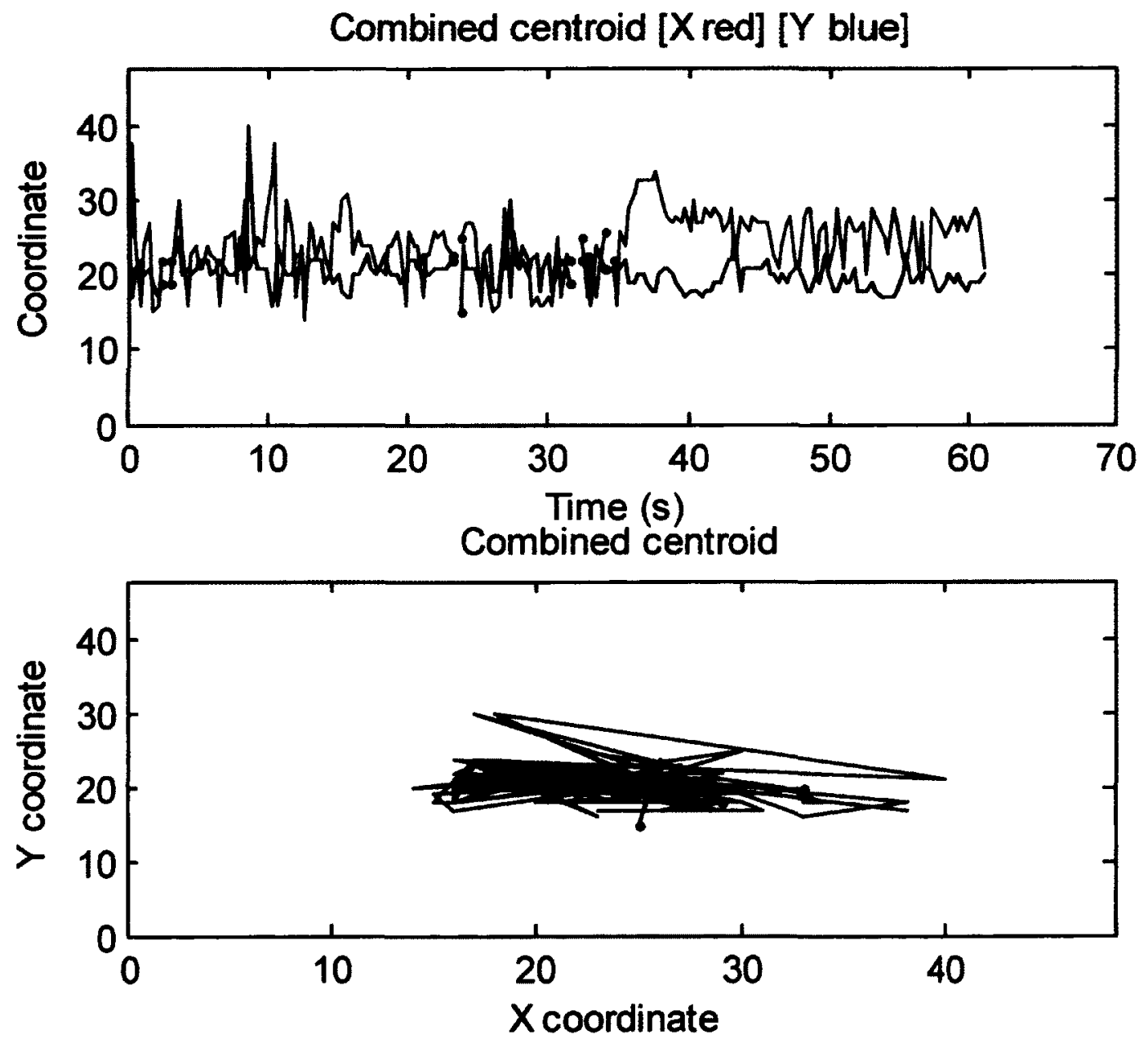

Figure 53: Example of COP over time for an older adult with standing stability issues. 

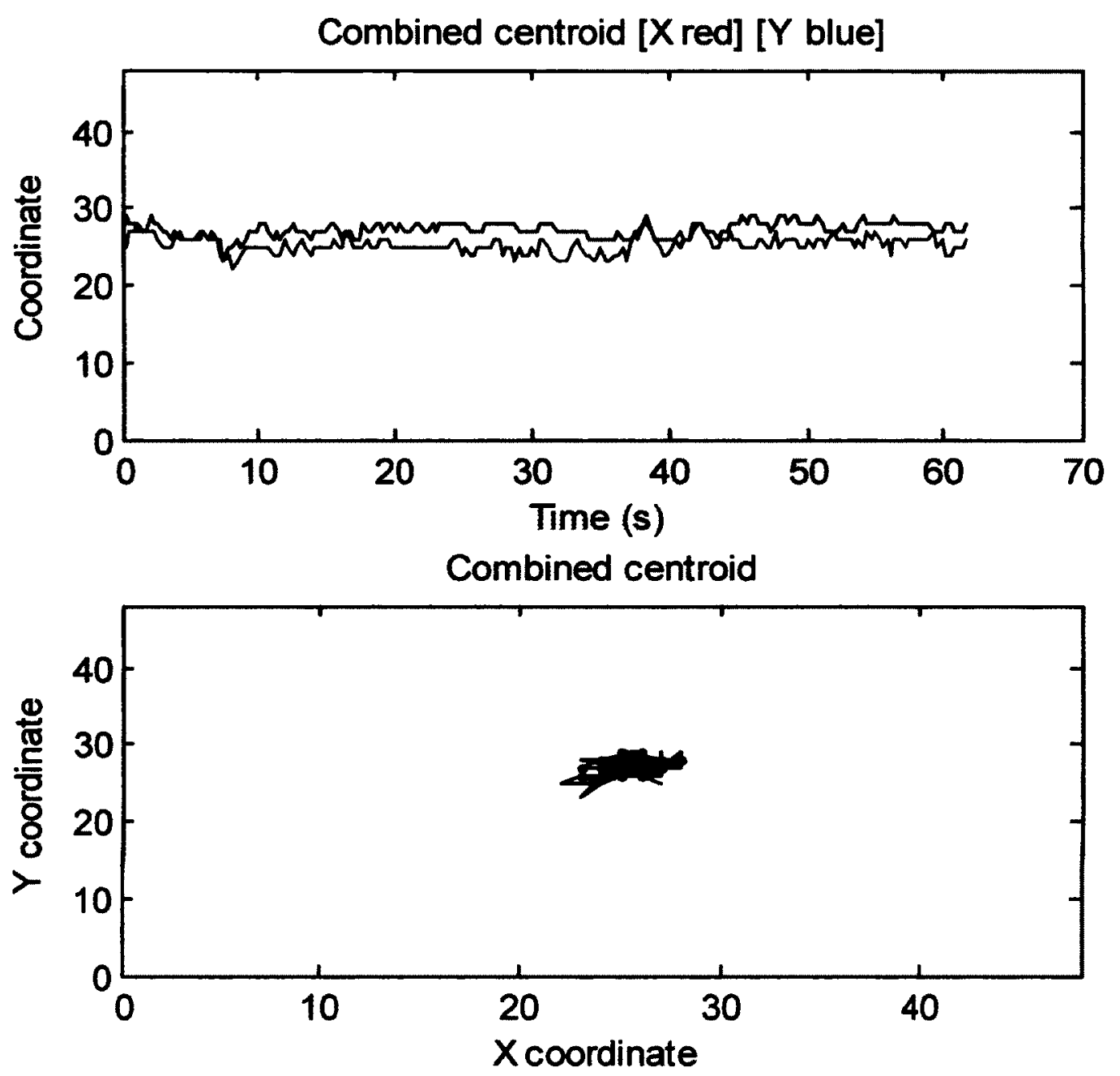

Figure 54: Example of COP over time for a young adult without standing stability issues

\subsubsection{Older adult compared to younger adult}

The clinical features can be plotted over time to assess whether the patient's standing stability has improved. The ratio of weight under each foot or amount of lean for each recording has been plotted in Figure 55. We see that the older adult always exerts more pressure under their left foot. There was no significant improvement over the two week trial. 
The average weight under each foot was calculated over the 10 recordings to give a result of $74 \%$ and $26 \%$ under the left and right foot respectively. A healthy individual should have an even amount of weight [38]; 50\% under each foot, which was confirmed with the data obtained from the young healthy adult.

The pressure distribution under each foot for a healthy individual should be even with the COP in the center of the footprint to maintain balance, as shown in Figure 52. Even pressure distribution under each foot was observed in the young healthy adult, however the older adult's COPs were slightly anterior to the center of the foot, as shown in Figure 56.

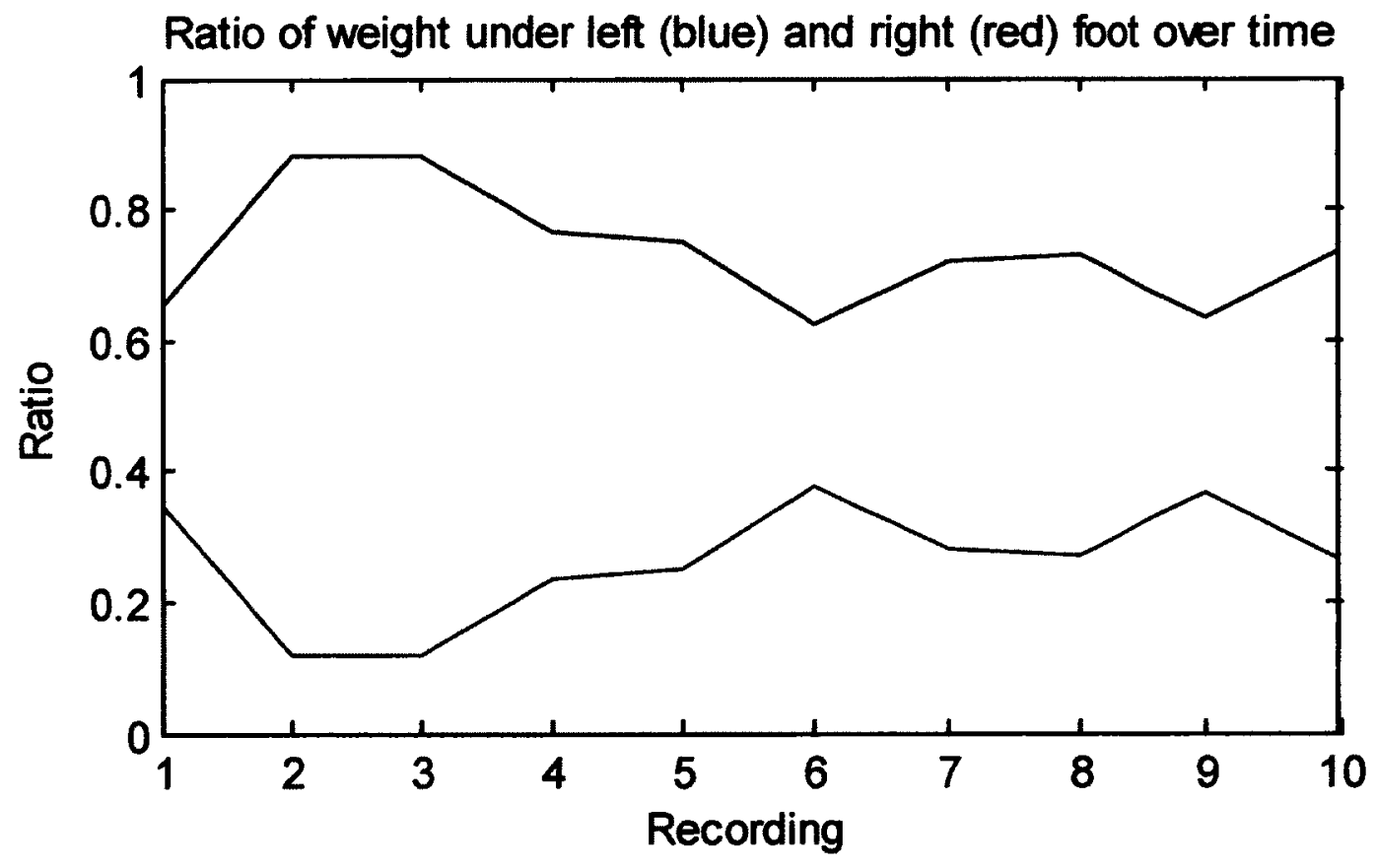

Figure 55: Amount of lean observed over time 


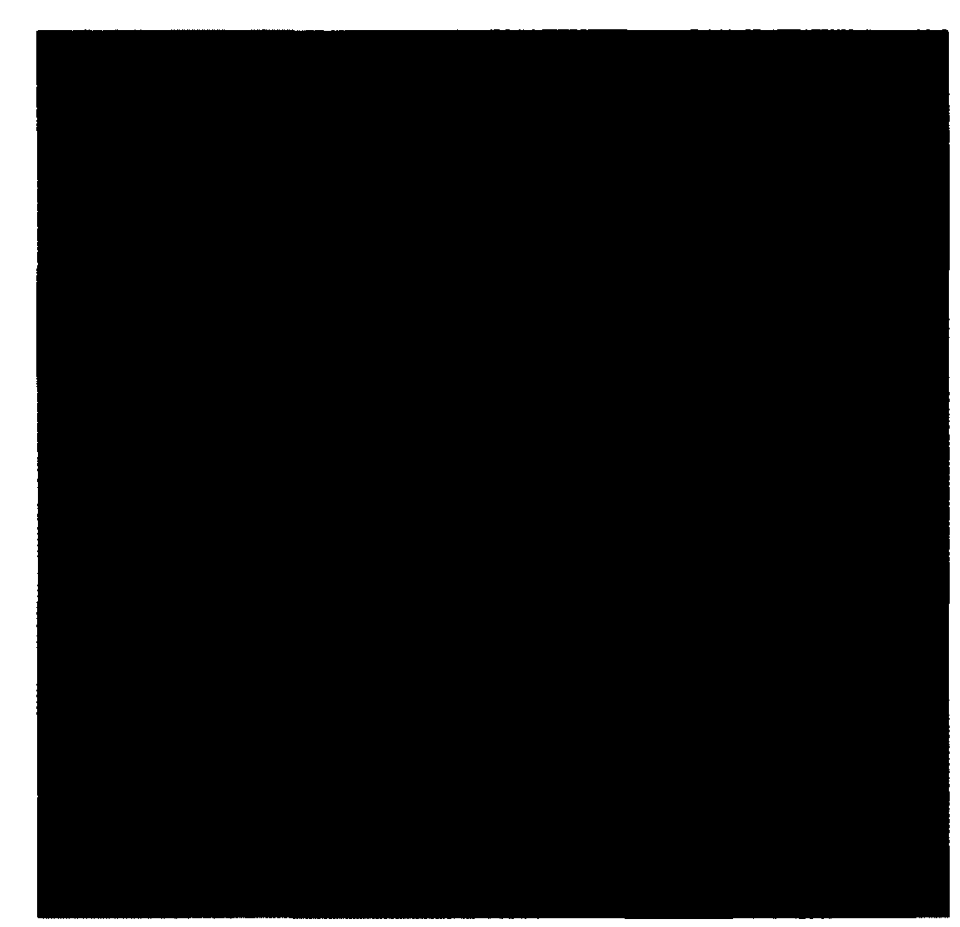

Figure 56: Older adult standing on sensor array. Calculated center of pressures shown illustrating an asymmetrical stance with greater weight on the left foot.

The third clinical feature was averaged over the 10 recordings resulting in a sway area of 360 $\mathrm{cm}^{2}$. For comparison, the healthy young adult had a sway area of $40 \mathrm{~cm}^{2}$.

The forth clinical feature, the speed of weight shifting was also different between the two participants. The average and maximum speed recorded in the older adult was $21 \mathrm{~cm} / \mathrm{s}$ and 54 $\mathrm{cm} / \mathrm{s}$, respectively. In the young adult, the corresponding values were $54 \mathrm{~cm} / \mathrm{s}$ and $89 \mathrm{~cm} / \mathrm{s}$ for the average and maximum values, respectively. 


\subsubsection{Comparison and discussion}

The four clinical features extracted from the pressure sensor technology provide quantitative information about stability measures that may assist a clinician in their assessment of a patient's standing stability. In addition to documenting stability, the clinician may be able to use this information to help personalize a treatment plan for the patient. Data recorded over time will provide the clinician with quantification of important parameters that contribute to clinical decisions regarding changes over time that could be due to intervention or physiological deterioration. This information could be used to improve the treatment program.

\subsubsection{Conclusion}

Using pressure sensitive floor sensors movements from a person standing on the sensor can be detected. Using the standing stability processing algorithm these movements are analyzed and important clinical features can be extracted. The clinical features are valuable measurements that contribute to the clinical profile when assessing the patient's standing stability. The ability to assess their standing stability will help to personalize treatment plans and improve recovery times for patients. Further research is being conducted to determine normal ranges of lean, area of sway, and movement velocity. It is the hope of the researchers to be able to classify people into levels of risk for falls based on their standing stability. 


\subsection{Bed sensor results}

The bed sensors have been installed into multiple patient homes for long term recording. The recorded data was processed using the algorithms discussed previously and clinical information was extracted. Using the long term recordings we were able to compare the clinical variables from an early recording to a recording approximately 6 months later. The patient reported they felt healthy at this time compared to earlier in the year. We found there was improvement in the clinical variables. We also analyzed the clinical features of a healthy older adult over a month to determine the variability of the clinical features extracted. These results will be discussed.

\subsubsection{Healthy older adult}

A healthy older male (64 years old) living in a senior's apartment consented to take part in a home monitoring study. The participant is a high functioning and very mobile senior citizen. The bed sensor was installed and data was recorded on a continuous basis for 23 days. The data was processed using the custom algorithms and visual inspection of pressure videos to determine the lie-to-sit (LtS), sit duration, and sit-to-stand (StS) time for the first transfer out of bed each morning.

The subject got up and out of bed on average at 9:17 AM the range was quite wide from 5:47 AM to 10:56 AM. Figure 57 shows the get up times for each day. His total transfer times were plotted for each day in Figure 58, the average was $71.8 \pm 68.7 \mathrm{~s}$. The phases of the bed exit were calculated, the LtS time for each day was plotted in Figure 59, and the average time for this phase was $16.4 \pm 7.3 \mathrm{~s}$. The amount of time he spend sitting between the LtS and StS phases was 
measured and plotted for each day in Figure 60 , the average time spent sitting was $49.8 \pm 65.9 \mathrm{~s}$. The final phase of the transfer, the StS time was plotted for each day in Figure 61, the average time was $5.6 \pm 2.6 \mathrm{~s}$. The bed exit times are summarized in Table 17.

Table 17: Bed exit timings for older adult

\begin{tabular}{|l|l|l|}
\hline Phase & Mean (s) & SD (s) \\
\hline Lie-to-Sit (LtS) & 16.4 & 7.3 \\
\hline Sitting & 49.8 & 65.9 \\
\hline Sit-to-Stand (StS) & 5.6 & 2.6 \\
\hline TOTAL & 71.8 & 68.7 \\
\hline
\end{tabular}

Get Up Time

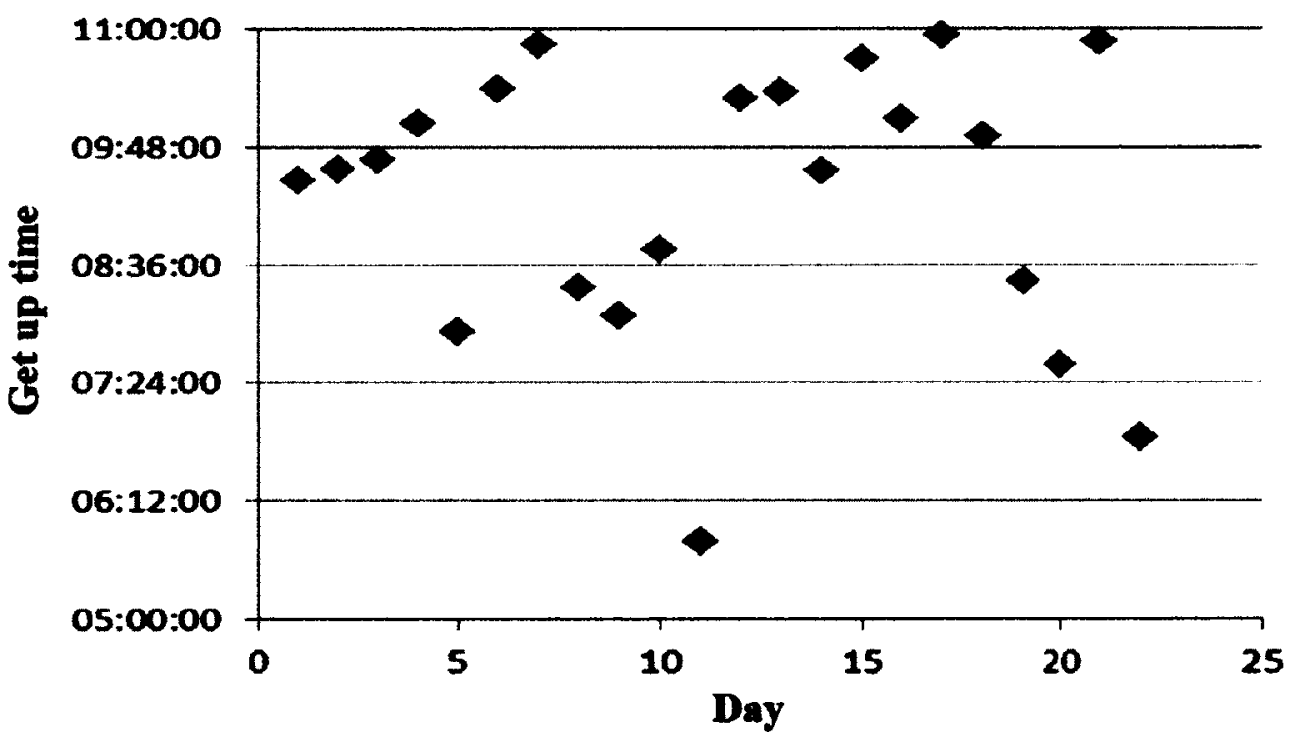

Figure 57: Get up time of older adult 


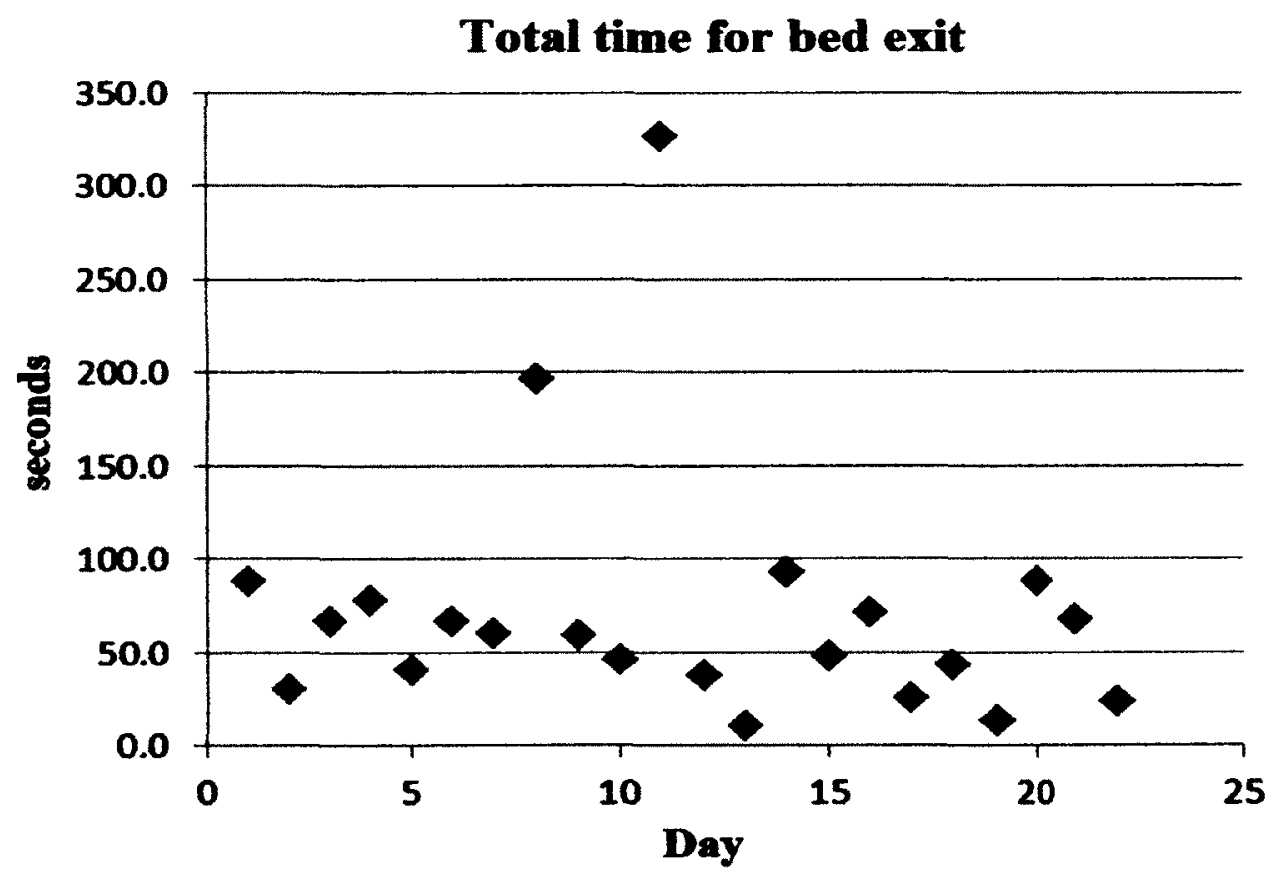

Figure 58: Total time for bed exit

LtS: Time to sit up from a lying position

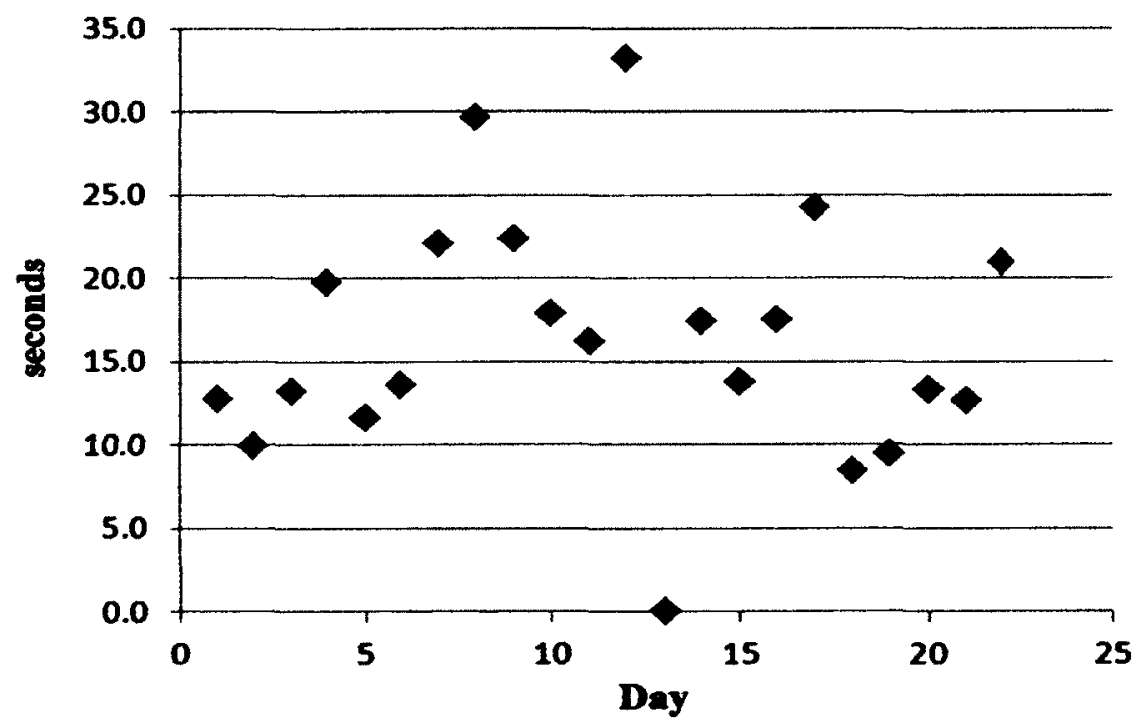

Figure 59: Time to sit up from a lying position 
Time spent in the sitting position

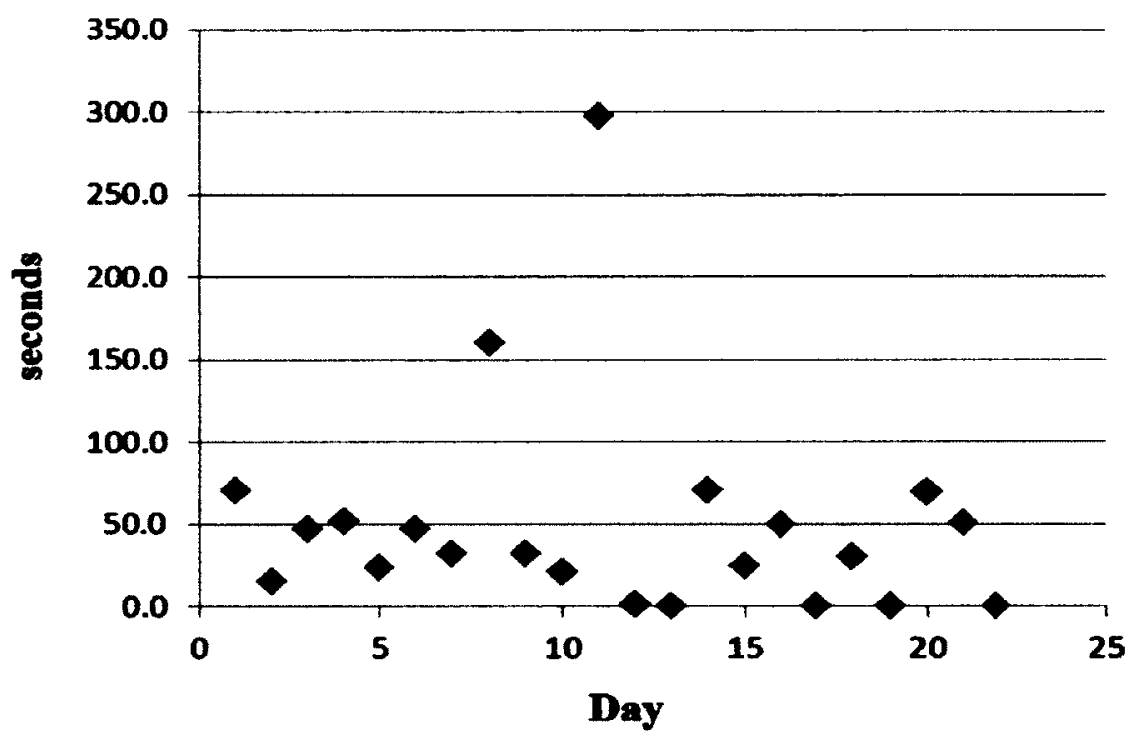

Figure 60: Time spent in the sitting position

StS: Time to stand up from sitting position

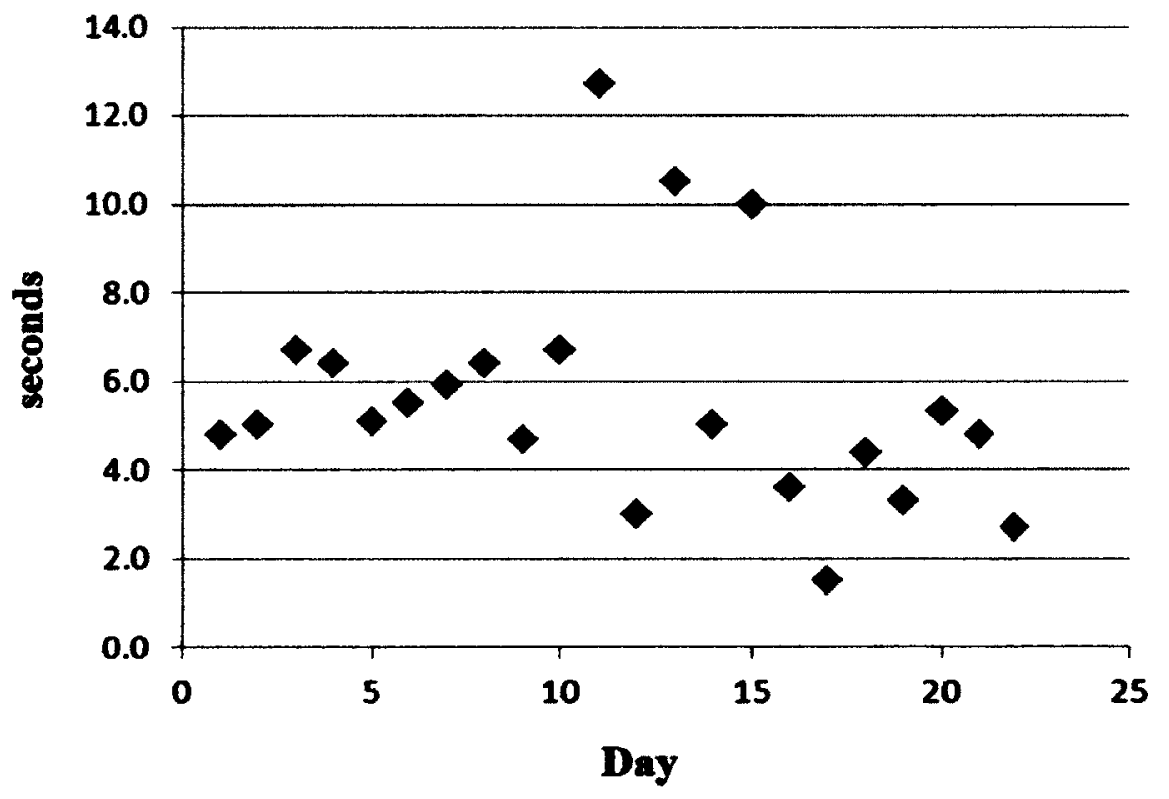

Figure 61: Time to stand up from sitting position 
We believe this is the first time longitudinal bed transfer timing data has been collected in an older adult in their home. This level of variability in morning transfers will create challenges in designing an automated mobility change detector.

\subsubsection{Frail older adult}

A frail older female ( 79 years old) living in a senior's apartment consented to take part in a home monitoring study between December 2011 and June 2012. The bed sensor was installed and data were recorded on a continuous basis. The data were processed using the custom algorithms and visual inspection of pressure videos to determine the sit-to-stand time for the first transfer out of bed each morning. Standardized functional mobility measures were recorded on 4 occasions between December 2011 and June 2011. These included gait speed over 5 metres, the Timed Up \& Go test (TUG), Berg Balance Scale and strength (triceps, grip, quadriceps). The participant also kept a critical event diary in which she recorded falls, hospital visits and changes in mobility.

All clinical measures indicated that this participant had improved functionally during the recording period. These are shown in Figure 62. The Berg Balance Scale score increased from $25 / 56$ to $35 / 56$, gait speed from $0.45 \mathrm{~m} / \mathrm{sec}$ to $0.72 \mathrm{~m} / \mathrm{sec}$, grip strength from $12 \mathrm{~kg}$ to $20 \mathrm{~kg}$ and her TUG decreased from $30 \mathrm{sec}$ to $18 \mathrm{sec}$. The pressure mat data was analyzed and the bed exit timings were extracted. The average time of each of the phases was calculated and plotted. Figure 63 shows the average time for the LtS phase. Figure 64 shows the average time for the sitting phase. Figure 65 shows the average time for the StS phase. Her initial StS transfer times were significantly longer than her final transfer times; $4.37 \pm 0.71 \mathrm{~s}$ (Winter 2011) vs. $2.53 \pm$ 
$0.98 \mathrm{~s}$ (Spring 2012). The improvement in StS time could be attributed in part by a biweekly exercise program the participant began attending.

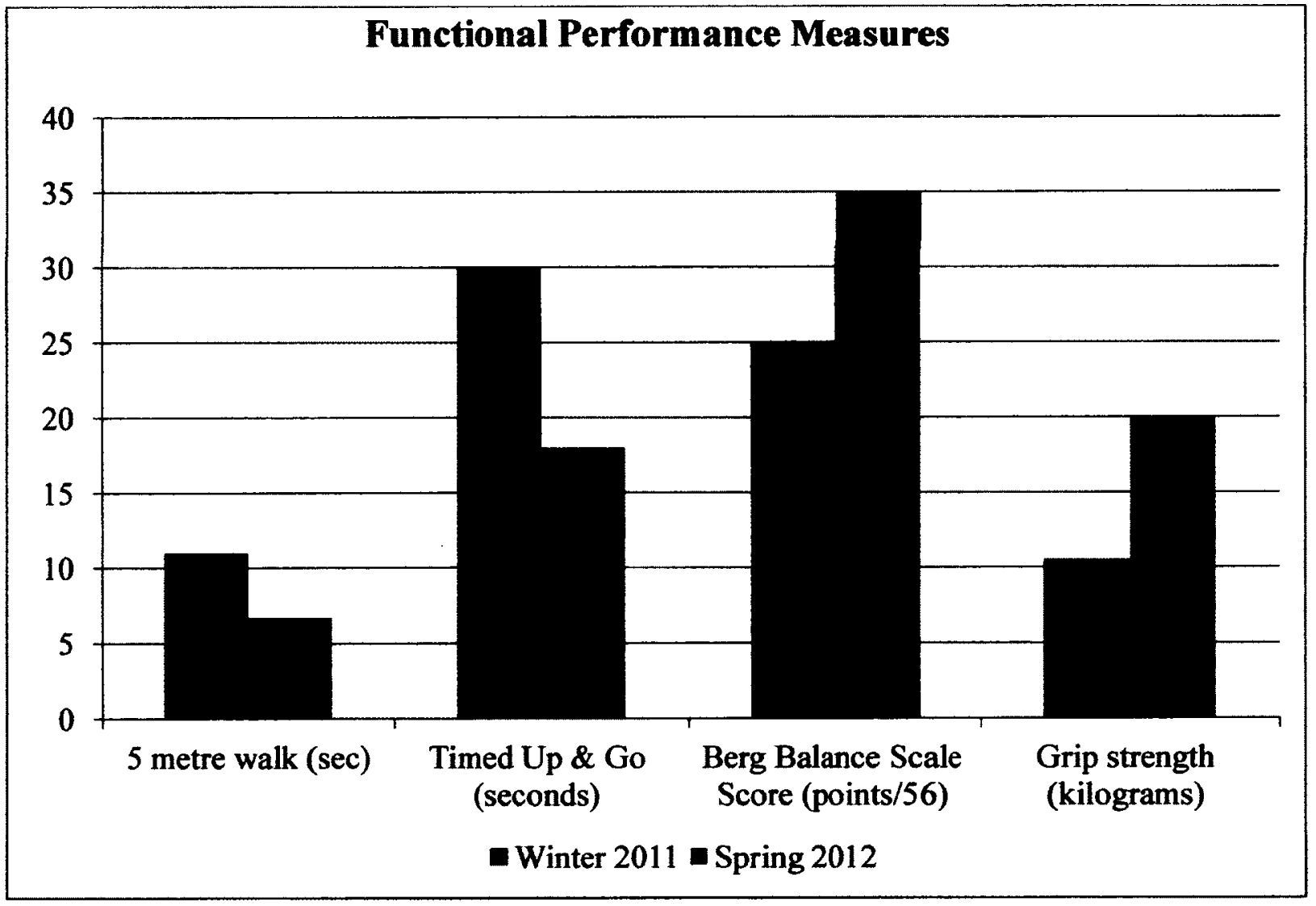

Figure 62: Functional Performance Measures 


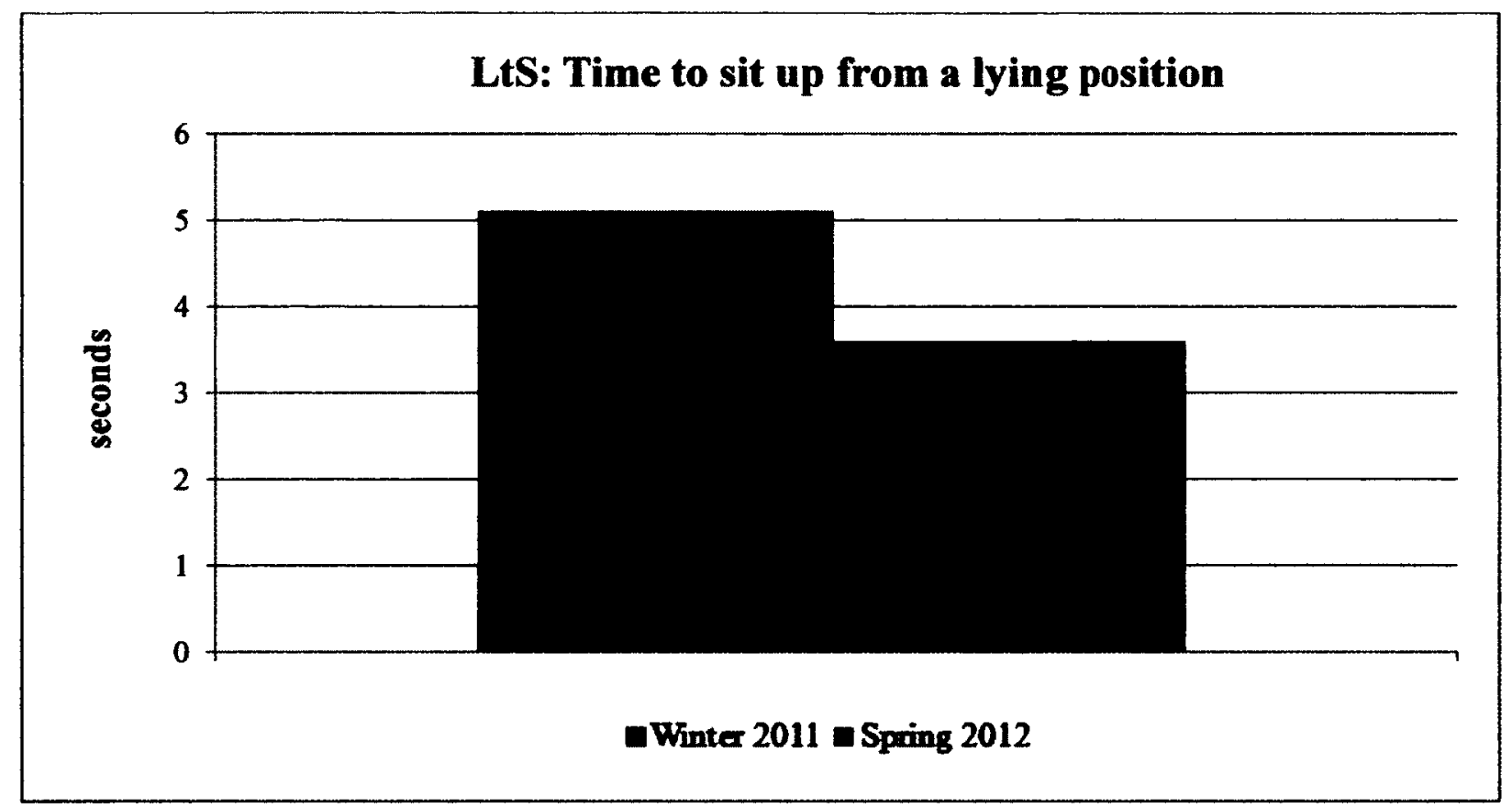

Figure 63: Time to sit up from a lying position

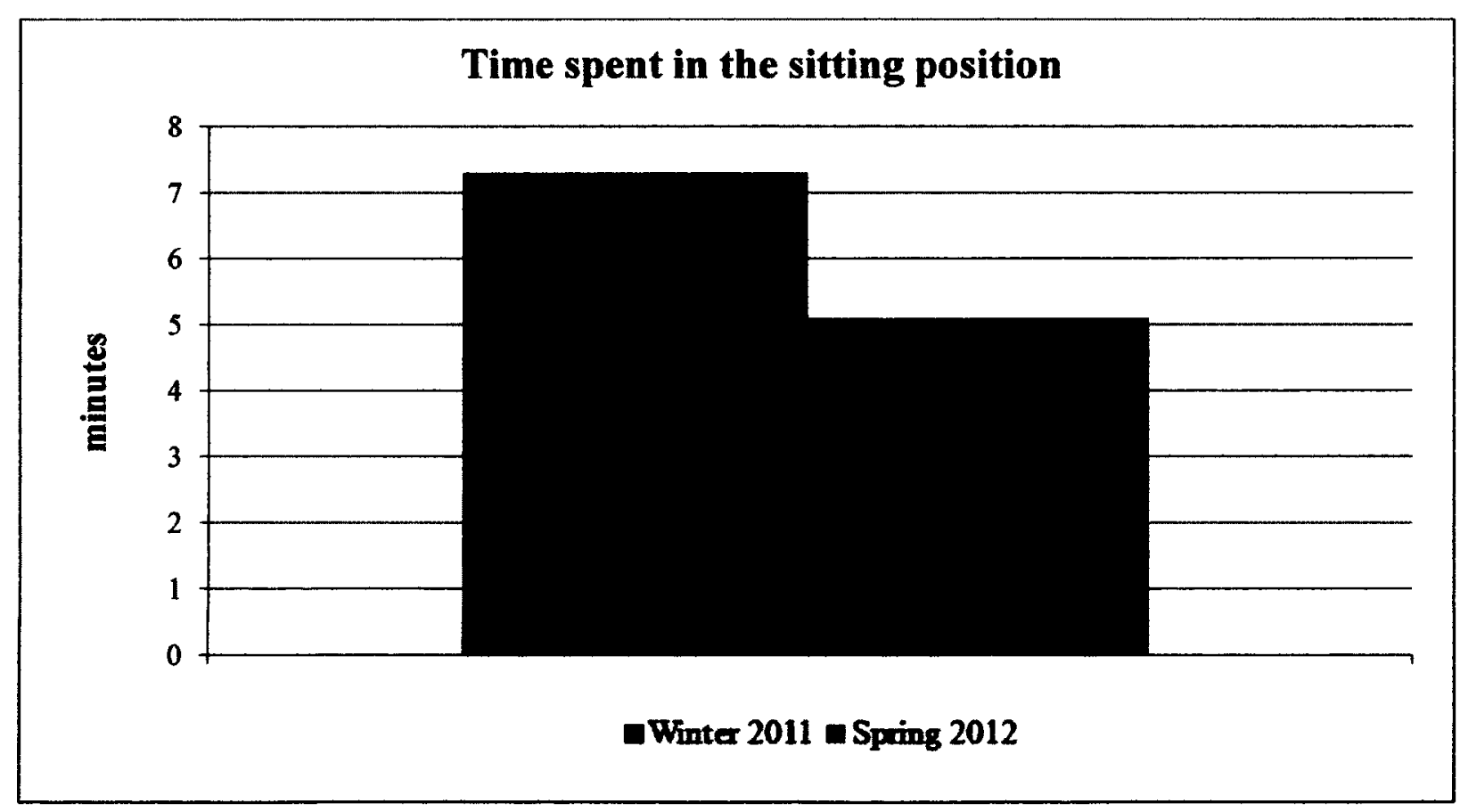

Figure 64: Time spent in the sitting position 


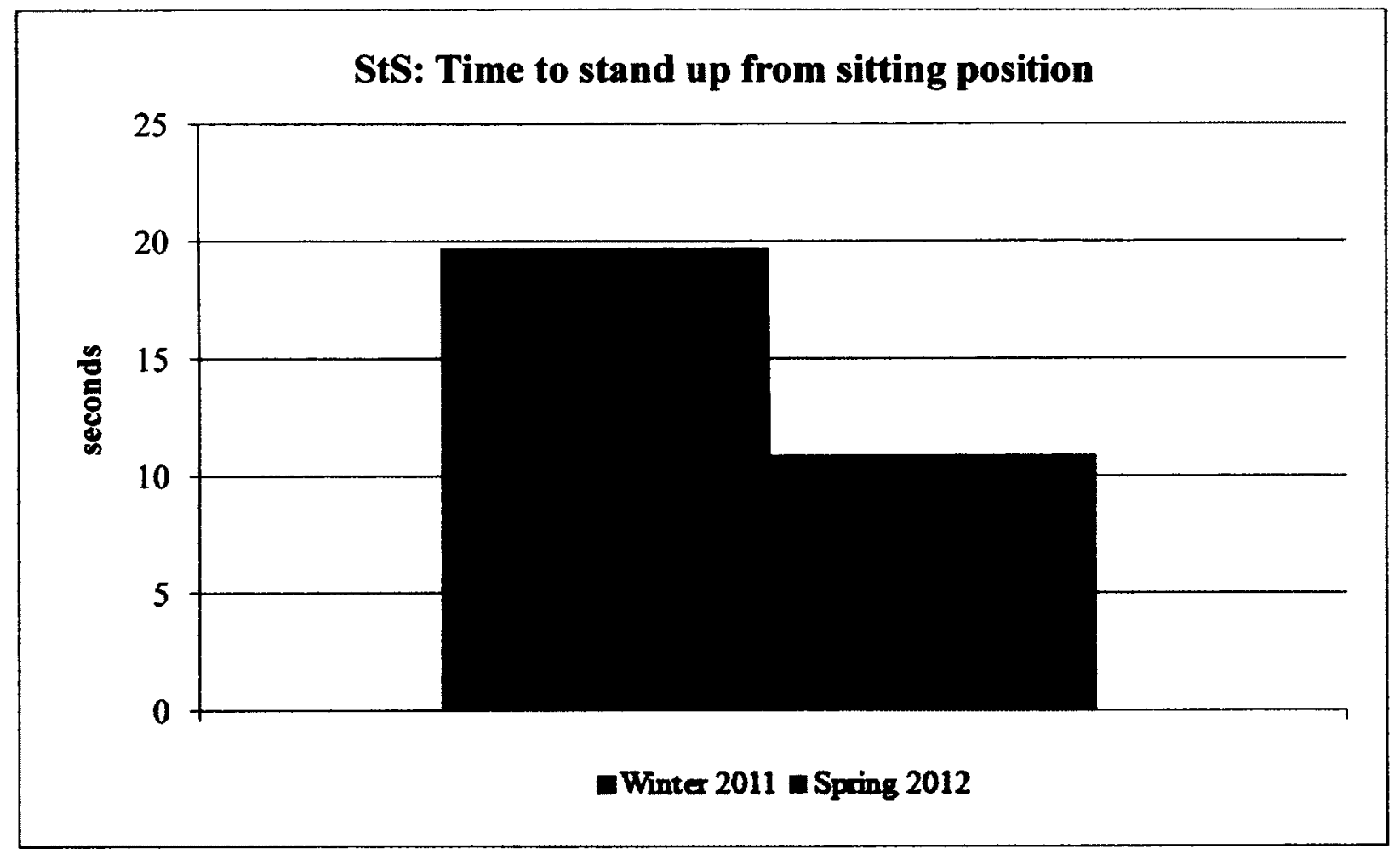

Figure 65: Time to stand up from sitting position

The pressure sensitive mat was able to detect changes in sit-to-stand transfer times that reflect a functional improvement over time. We believe this is the first time longitudinal bed transfer timing data have been collected from an older adult in their home. Further analysis of the mat data pre and post critical incidents will determine whether changes in bed transfer patterns can be used as a fall prevention tool.

\subsubsection{Comparison}

While the purpose of the research was not to compare clinical measures between patients it is interesting to see the difference between the healthy and the frail older adults. We noticed the 
healthy individual has significantly more variability in their bed exits, while the frail adult is more consistent. This could be explained by the fact that the healthy individual has the freedom to jump out of bed quickly or they may choose to slowly get up. The frail individual does not have the option to jump out of bed quickly; they consistently require more time to perform the lie-to-sit and the sit-to-stand transfer. We also notice that the individual almost always requires some time to sit after performing the LtS before they are able to perform StS.

\subsection{Combination of sensors}

This thesis has shown it is possible to extract clinical information from floor tile based pressure sensors to help assess the standing stability of older adults. It has also shown that bed based pressure sensors can be used to extract sit-to-stand times which help to assess mobility. These sensors can be installed in a patient's home to unobtrusively monitor them. Using data extracted from both sensors we can get a better assessment of the patient's mobility and stability. These measurements can be taken on a daily basis and any abnormalities could be detected. It is important not to rely on a single source of information in patient monitoring; therefore it would be beneficial to incorporate both the floor tile and the bed mat pressure sensors into the smart home environment. 


\section{Chapter 6: Conclusion}

\subsection{Summary of work}

This section summarizes the work involved in completing the thesis objectives. Pressure signal data was recorded using both floor tile and bed mat technologies from various participants. The pressure signals were examined and relevant signal features were found. Relevant clinical information that could be extracted from signal features was determined, this included lie-to-sit and sit-to-stand times, and static and dynamic standing stability and symmetry. The best set of features for extracting the clinical information was found. An automated pressure signal processing algorithm was designed and implemented in MATLAB. A GUI was developed for each algorithm. Finally the performance of system was evaluated.

\subsection{Thesis contributions}

The following contributions were made during the thesis research. 
- A novel processing algorithm was developed to convert raw pressure data recorded by the floor pressure sensor into pressure mapped images for clinical analysis. Various sampling rates and resolutions were tested to determine minimum requirements to obtain high accuracy in the least amount of processing time [6]. The work was published in IEEE International Instrumentation and Measurement Technology Conference 2012 (I2MTC 2012) in Graz, Austria, May 2012.

- A standing stability system was designed using the original processing algorithm with the addition of a secondary one to extract clinical features that can assist in assessing a patient's standing stability. The system analyzes features related to static balance; the amount of lean, the center of pressure, the amount of sway while standing still and dynamic balance; the velocity at which they can shift their weight. The standing stability of an older adult with chronic stroke was assessed and compared to a healthy young adult [7]. The system and results were published in IEEE International Symposium on Medical Measurements and Applications Proceedings (MeMeA 2012) in Budapest, Hungary, May 2012.

- A MATLAB graphical user interface (GUI) was developed to assist researchers in analyzing the floor pressure sensor recordings. The GUI allows for quick processing with all options for data output easily changed. 
- A new pressure sensitive mat was tested in a pilot project to assess its usability for long term bed pressure recordings. Data was processed and the quality was assessed. The mat was considered more than adequate for our research purposes. The work will be presented at the Regional Geriatric Conference in Ottawa, Ontario, October 2012 [8].

- A novel processing algorithm was developed to extract meaningful clinical information from the pressure mat recordings. The processing algorithm is able to scan through $100 \mathrm{~s}$ of hours of recordings and extract meaningful information automatically and display that in a user friend way. One of the clinical features is the bed exit timing, data collected from an older adult was analyzed and the results will be presented at the Canadian Association on Gerontology Conference in Vancouver, British Columbia, October, 2012 [9].

- A MATLAB graphical user interface (GUI) was developed to assist researchers in analyzing the bed mat pressure sensor recordings. The GUI allows for quick processing with all options for data output easily changed.

\subsection{Future work}

Patient monitoring in this thesis used real world recordings from participants in their own home. We believe this is the first time pressure sensor arrays were used to extract clinical information 
related to mobility from patients in their home. More research needs to be done using real world data and environments. Other clinical information can still be extracted from the recordings.

Bed mat pressure sensors can be used to assess the quality of a patient's sleep. This could be based on movements such as tossing and turning. Other movements such as muscle spasms or restless leg syndrome, or Parkinson's could be detected from pressure mat recordings. Other sleep disorders like sleep apnea could be assessed based on arousals detected. Additional sensors could be used such as microphone array to detect coughing related to sleep apnea.

The smart home could use multiple floor sensor arrays, this could not only provide standing stability assessments, but also gait analysis. Walking and running could be analyzed from real world data. Pressure sensor arrays could also be embedded in shoes to perform patient monitoring while the patient is out of the home.

There is much to be learned in mobility monitoring and its use in smart homes. 


\section{References}

[1] Human Resources and Skills Development Canada. "Canadians in Context - Aging Population" Internet: http://www4.hrsdc.gc.ca/.3ndic.1t.4r@-eng.jsp?iid=33, Sept. 30, 2012 [Sept. 30, 2012].

[2] Comfort Life. "Aging at home." Internet: http:/www.comfortlife.ca/aging-at-home.php, [Sept. 30, 2012].

[3] M. Holtzman, A. Arcelus, R. Goubran, F. Knoefel, "A Pressure Sensitive Home Environment," in Proc. of IEEE International Workshop on Haptic Audio Visual Environments and their Applications, Ottawa, ON, Canada, 2006, pp. 10-14.

[4] M. Holtzman, A. Arcelus, R. Goubran, F. Knoefel, "Breathing Signal Fusion in Pressure Sensor Arrays," in Proc. of IEEE International Workshop on Medical Measurements and Applications, Ottawa, ON, Canada, 2008, pp. 71-76.

[5] A. Arcelus, C. Herry, R. Goubran, F. Knoefel, H. Sveistrup, M. Bilodeau, "Determination of Sit-to-Stand Transfer Duration Using Bed and Floor Pressure Sequences." IEEE Transactions on Biomedical Engineering, vol. 56(10), pp. 2485-2492, Jul. 2009. 
[6] M. Taylor, R. Goubran, F. Knoefel, "Patient standing stability measurements using pressure sensitive floor sensors," in Proc. of IEEE International Instrumentation and Measurement Technology Conference, Graz, Austria, 2012, pp. 1275-1279.

[7] M. Taylor, D. McEwen, R. Goubran, H. Finestone, F. Knoefel, H. Sveistrup, M. Bilodeau, "Assessing standing stability of older adults using pressure sensitive arrays," in Proc. of IEEE International Symposium on Medical Measurements and Applications, Budapest, Hungary, 2012, pp.1-5.

[8] F. Knoefel, M. Taylor, T. Grant, S. Rocan, J. Jutai, H. Sveistrup, R. Goubran, J. Taylor, M. Bilodeau, "The use of a pressure-sensitive mat to monitor bed transfer changes in an older adult," Annual General Meeting Regional Geriatric Program of Eastern Ontario, Ottawa, Canada, 2012.

[9] F. Knoefel, M. Taylor, T. Grant, S. Rocan, J. Jutai, H. Sveistrup, R. Goubran, J. Taylor, M. Bilodeau, "Unobtrusive Home Bed Transfer Monitoring in an Older Adult," 41 st Annual Scientific and Educational Meeting Canadian Association on Gerontology, Vancouver, Canada, 2012.

[10] M. Edmonds. "How Smart Homes Work," Internet: http:/home.howstuffworks.com/smarthome.htm, [Sept. 30, 2012].

[11] E.D. Williams, "Scoping the potential of monitoring and control technologies to reduce energy use in homes," in Proc. of IEEE International Symposium on Electronics \& the Environment, Orlando, FL, USA, 2007, pp. 239-244. 
[12] D. Stefanov, Z. Bien, W. Bang, "The smart house for older persons and persons with physical disabilities: structure, technology arrangements, and perspectives," IEEE Transactions on Neural Systems and Rehabilitation Engineering, vol. 12(2), pp. 228-250, June 2004.

[13] Bruyère Continuing Care. "Our Mission," Internet: http://www.Bruyère.org/en/our-mission, [Sept. 30, 2012].

[14] TAFETA Smart Systems for Health. "Technology Tours." Internet: http://www.tafeta.ca [Sept. 30, 2012].

[15] M. Nevitt, S. Cummings, E. Hudes, "Risk Factors for Injurious Falls: a Prospective Study," Journal of Gerontology, vol. 46(5), pp. 164-170, Feb. 1991.

[16] P. Millington, B. Myklebust, G. Shambes, "Biomechanical analysis of the sit-to-stand motion in elderly persons," Archives of Physical Medicine and Rehabilitation, vol. 73(7), pp. 609-617, Jul. 1992.

[17] W. Janssen, H. Bussmann, H. Stam, "Determinants of the Sit-to-Stand Movement: A Review," Journal of the American Physical Therapy Association, vol. 82(9), pp. 866-879, Sept. 2002.

[18] D. Weeks, R. Forget, L. Mouchnino, D. Gravel, D. Bourbonnais, "Interaction between Attention Demanding Motor and Cognitive Tasks and Static Postural Stability," International Journal of Experimental and Clinical Gerontology, vol. 49(4), pp. 225-232, 2003. 
[19] I. Melzer, N. Benjuya, J. Kaplanski, "Postural stability in the elderly: a comparison between fallers and non-fallers" Oxford Journal of Age and Ageing, vol. 33(6), pp. 602-607, Jun. 2004.

[20] H. Yack, R. Berger, "Dynamic Stability in the Elderly: Identifying a Possible Measure," Oxford Journal of Gerontology, vol. 48(5), pp. 225-230, Dec. 1992.

[21] J. Raymakers, M. Samson, H. Verhaar, "The assessment of body sway and the choice of the stability parameter(s)," Journal of Gait \& Posture, vol. 21(1), pp. 48-58, Jan. 2005.

[22] M. Rogers, M. Mille, "Lateral Stability and Falls in Older People" Exercise \& Sport Sciences Reviews, vol. 31(4), pp. 182-187, Oct. 2003.

[23] A. Andrews, R. Bohannon, "Distribution of muscle strength impairments following stroke," Journal of Clinical Rehabilitation, vol. 14(1), pp. 79-87, Jan. 2000.

[24] H. Hoenig, H. Siebens, "The American Geriatrics Society: Geriatric Rehabilitation" Internet: http://newfrontiers.americangeriatrics.org/chapters/chapter12.html [Sept. 30, 2012]

[25] K. Daley, N. Mayo, S. Wood-Dauphinée, "Reliability of Scores on the Stroke Rehabilitation Assessment of Movement (STREAM) Measure," Journal of the American Physical Therapy Association, vol. 79(1), pp. 8-23, Jan. 1999.

[26] X. Tortolero, K. Masani, T. Thrasher, M. Popovic, "Instability Prediction by Monitoring Center of Pressure During Standing," in Proc. of IEEE Engineering in Medicine and Biology Society Annual International Conference, New York, NY, USA, 2006, pp. 5412-5415. 
[27] J. Fiolka, Z. Kidon, "Center of pressure trajectory segmentation for postural stability analysis," in Proc. of IEEE International Mixed Design of Integrated Circuits and Systems Conference, Gliwice, Poland, 201 1, pp. 39-43.

[28] D. Hewson, N. Singh, H. Snoussi, J. Duchene, "Classification of elderly as fallers and nonfallers using centre of pressure velocity," in Proc. of IEEE International Conference of Engineering in Medicine and Biology Society, Buenos Aires, Argentina, 2010, pp. 36783681 .

[29] P. Mostarac, R. Malaric, M. Jurcevic, H. Hegedus, A. Lay-Ekuakille, P. Vergallo, "System for monitoring and fall detection of patients using mobile 3-axis accelerometers sensors," in Proc. of IEEE International Workshop on Medical Measurements and Applications, Bari, Italy, 2011, pp. 456-459.

[30] R. Mayagoitia, J. Lotters, P. Veltink, "Standing stability evaluation using a triaxial accelerometer," in Proc. of IEEE International Conference of Engineering in Medicine and Biology Society, Amsterdam, Netherlands, 1996, pp. 573-574.

[31] D. Townsend, R. Goubran, M. Frize, F. Knoefel, "Measuring chest movement using an array of unobtrusive pressure sensors," in Proc. of IEEE Instrumentation and Measurement Technology Conference, Austin, TX, USA, 2010, pp. 1053-1056.

[32] D. Townsend, M. Holtzman, R. Goubran, M. Frize, F. Knoefel, "Relative Thresholding With Under-Mattress Pressure Sensors to Detect Central Apnea," IEEE Transactions on Instrumentation and Measurement, vol. 60, no. 10, pp. 3281-3289, Oct. 2011. 
[33] H.F. Van der Loos, N. Ullrich, H. Kobayashi, "Development of Sensate and Robotic Bed Technologies for Vital Signs Monitoring and Sleep Quality Improvement," Autonomous Robots, vol. 15, no. 1, pp. 67-79, July 2003.

[34] D. Rosenbaum, H. Becker, "Plantar pressure distribution measurements. Technical background and clinical applications," Foot and Ankle Surgery, vol. 3, no. 1, pp. 1-14, Mar. 1997.

[35] E. Reimer, L. Baldwin, "Cavity sensor technology for low cost automotive safety \& control devices," Air Bag Technology '99, Cobo Convention Center, Detroit, USA, 1999. [Online] Available: http://www.canpolar.com/principles.shtm. [Sept. 24, 2012].

[36] I. Veledar, A. Arcelus, R. Goubran, F. Knoefel, H. Sveistrup, M. Bilodeau, "Sit-to-stand timing measurements using pressure sensitive technology," in Proc. of IEEE Instrumentation and Measurement Technology Conference, Austin, TX, USA, 2010, pp. 1337-1340.

[37] A. Arcelus, M. Holtzman, I. Veledar, R. Goubran, H. Sveistrup, P. Guitard, "Contact Location Estimation from a Nonlinear Array of Pressure Sensors," in Proc. of IEEE Instrumentation and Measurement Technology Conference, Victoria, BC, Canada, 2008, pp. 1969-1973.

[38] G. Thibodeau, K. Patton, The Human Body in Health and Disease. 2nd ed. St. Louis, MO: Mosby-Year Book, Inc. 1997.

[39] D. Townsend. "Context-aware Algorithms for Sleep Apnea Monitoring and Sensor Acceptance using Unobtrusive Pressure Sensors Arrays" Ph.D. thesis, Carleton University, Canada, 2012. 
[40] M. Howell-Jones. "Autonomous Patient Monitoring with a Pressure Sensor Array." M.A.Sc. thesis, Carleton University, Canada, 2006.

[41] I. Veledar. "Patient Bed Transfer Analysis Using Pressure Sensor Arrays." M.A.Sc. thesis, Carleton University, Canada, 2009.

[42] A. Arcelus. "Context-Aware Smart Home Monitoring Through the Analysis of Pressure Sequences." Ph.D. thesis, Carleton University, Canada, 2010.

[43] N. Foubert. "Posture Recognition and Postural Transition Detection Using Bed-Based Pressure Sensor Arrays." M.A.Sc. thesis, Carleton University, Canada, 2010.

[44] Tactex Controls Inc., "Floor Sensor Kits: Portable, Pressure Mapping Sensor Panels," Internet: http://cias.rit.edu/ nmtp/179773/teamkittycat/floor_sensor_kit.pdf [Oct 2, 2012]. 